\title{
Theory- and evidence-based interventions to enhance adherence among HIV-infected patients using highly active antiretroviral therapy
}

Citation for published version (APA):

de Bruin, M. (2009). Theory- and evidence-based interventions to enhance adherence among HIVinfected patients using highly active antiretroviral therapy. [Doctoral Thesis, Maastricht University]. Universitaire Pers Maastricht. https://doi.org/10.26481/dis.20091008mb

Document status and date:

Published: 01/01/2009

DOI:

10.26481/dis.20091008mb

Document Version:

Publisher's PDF, also known as Version of record

Please check the document version of this publication:

- A submitted manuscript is the version of the article upon submission and before peer-review. There can be important differences between the submitted version and the official published version of record.

People interested in the research are advised to contact the author for the final version of the publication, or visit the DOI to the publisher's website.

- The final author version and the galley proof are versions of the publication after peer review.

- The final published version features the final layout of the paper including the volume, issue and page numbers.

Link to publication

\footnotetext{
General rights rights.

- You may freely distribute the URL identifying the publication in the public portal. please follow below link for the End User Agreement:

www.umlib.nl/taverne-license

Take down policy

If you believe that this document breaches copyright please contact us at:

repository@maastrichtuniversity.nl

providing details and we will investigate your claim.
}

Copyright and moral rights for the publications made accessible in the public portal are retained by the authors and/or other copyright owners and it is a condition of accessing publications that users recognise and abide by the legal requirements associated with these

- Users may download and print one copy of any publication from the public portal for the purpose of private study or research.

- You may not further distribute the material or use it for any profit-making activity or commercial gain

If the publication is distributed under the terms of Article $25 \mathrm{fa}$ of the Dutch Copyright Act, indicated by the "Taverne" license above, 
Theory- and Evidence-based Interventions to

Enhance Adherence among HIV-infected Patients

using Highly Active Antiretroviral Therapy 
ISBN 9789052788609

The clinical studies described in this thesis have been conducted at the Academic Medical Center in Amsterdam, the Netherlands. Funding for the pilot-study in this thesis was provided by AARDEX Itd. (Switzerland). Materials for the randomized controlled trial were financed from internal budgets from the Academic Medical Center, Amsterdam. The Ph.D. position was created by the Faculty of Psychology, Maastricht University.

Abbott, Boehringer Ingelheim, Bristol-Myers Squibb, Gilead, and Janssen-Cilag, financially supported the printing and distribution of this thesis. The graphic for the cover was provided by GlaxoSmithKline. 
Theory- and Evidence-based Interventions to Enhance Adherence among HIV-infected Patients using Highly Active Antiretroviral Therapy

\author{
PROEFSCHRIFT
}

ter verkrijging van de graad van doctor aan de Universiteit Maastricht, op gezag van de Rector Magnificus, Prof. Mr. G.P.M.F. Mols, volgens het besluit van het College van Decanen,

in het openbaar te verdedigen op donderdag 8 oktober 2009 om 12.00 uur door

Marijn de Bruin,

geboren op 16 oktober 1977, te Amsterdam.

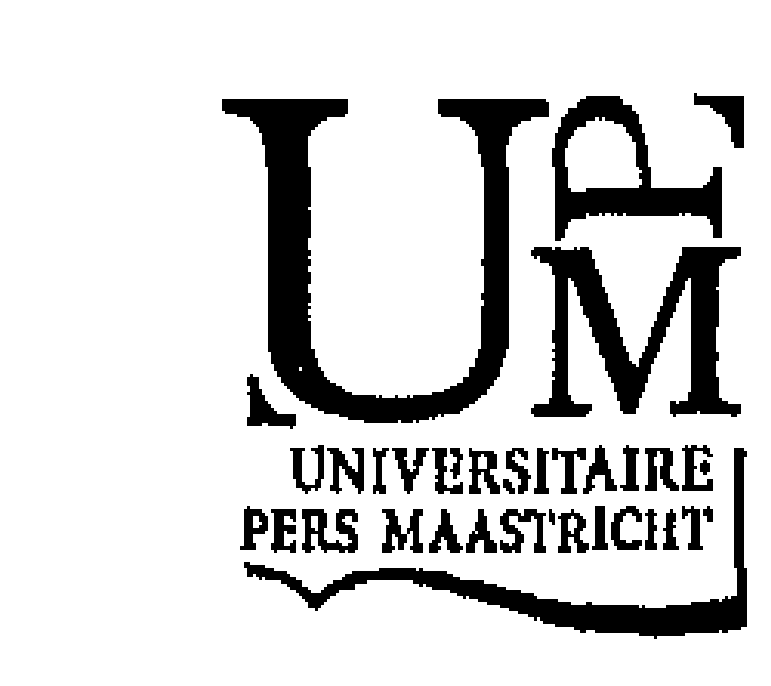




\section{Promotores}

Prof. dr. Harm J Hospers

Prof. dr. Gerjo Kok

\section{Copromotor}

Prof. dr. Jan M Prins (Academisch Medisch Centrum, Amsterdam)

\section{Beoordelingscommissie}

Prof. dr. Nanne K de Vries (voorzitter)

Prof. dr. L Kay Bartholomew (University of Texas, Houston; Universiteit Maastricht)

Dr. Arjan ER Bos

Dr. David M Burger (Radboud Universiteit Nijmegen)

Prof. dr. Bernadette M Jansma

Dr. Jan TM van der Meer (Academisch Medisch Centrum, Amsterdam) 
CONTENTS

$\begin{array}{lll}\text { CHAPTER } 1 & 7\end{array}$

CHAPTER 2 Theory- \& Evidence-based Intervention to Improve

Adherence to Antiretroviral Therapy among HIV-infected

Patients in the Netherlands: A Pilot-study

CHAPTER 3 Variability in Standard Care Quality of HAART-adherence

Studies: Implications for the Interpretation and Comparison

of Intervention Effects

CHAPTER 4 Electronic Monitoring-based Counseling to Enhance

Adherence among HIV-infected Patients using HAART:

51

A Randomized Controlled Trial

CHAPTER 5 How Intentions Translate into Behavior: Preparatory and Self-regulation Activities Mediate the Intention-Behavior Relation in a Longitudinal Study of Medication Adherence among HIV-infected Patients using HAART

CHAPTER 6 How adherence care impacts treatment outcomes in HIV: A meta-analysis of randomized controlled trials

CHAPTER 7 General Discussion 109

Supplemental Material

REFERENCES

SUMMARY

SAMENVATTING

ACKNOWLEDGEMENTS, DANKWOORD 
CHAPTER 1

Introduction 


\section{CHAPTER 1}

\section{INTRODUCTION}

The World Health Organization and the Joined United Nations Program on HIV/AIDS estimate that since HIV was first recognized on December 1, 1981, about 25 million people have died of AIDS. In 2007, about 33 million people worldwide were infected with HIV, 2.7 million people were newly infected with HIV, and 2 million people died of AIDS (Joined United Nations Programme on HIV/AIDS, 2007). Although subSaharan Africa is affected most by the pandemic, harboring approximately $67 \%$ of the HIV-infected people, HIV/AIDS is also a major public health problem in the United States and Europe. In the United States approximately 1.300 .000 people are infected with HIV in 2007 (0.6\% of the adult population in 2007), and in Western and Central Europe that number was estimated at $760.000(0.3 \%$ of the adult population (Joined United Nations Programme on HIV/AIDS, 2007). In 2007 alone approximately 46.000 people were newly infected in the United States, and about 31.000 in Central and Western Europe, a yearly incidence rate that has remained stable between 2001 and 2007 (Joined United Nations Programme on HIV/AIDS, 2008). In the Netherlands, where the clinical trials described in this thesis have been executed, approximately 18.000-20.000 people are currently infected with HIV $(0.2 \%$ of the adult population), and the yearly incidence rate lies around 1200 (EuroHIV, 2007). Considering these numbers of the HIV-pandemic worldwide, it is not an understatement that HIV has impacted the lives of many, and will affect the lives of many more in the future.

In rich countries, the face of HIV has changed dramatically since highly active antiretroviral therapy (HAART) was first introduced in 1996 . This combination of antiretroviral medicines does not cure people from HIV, but it can effectively suppress HIV so that patients can regain and maintain a healthy immune function. The introduction of HAART has led to a marked reduction in morbidity and mortality among HIV-infected patients in developed countries, and an increase in life expectancy (Mocroft et al., 2000; Moore \& Chaisson, 1999; Sendi et al., 1999). A recent study showed that the life expectancy of an HIV-positive patient at age 20 is currently estimated at 49.4 years (Antiretroviral Cohort Collaboration, 2008). Hence, although HIV-patients' are still expected to live shorter than the general population, access to HAART has had a great impact on patients' long-term health, and hopefully these results will be similarly impressive in those countries that have started to benefit from HAART more recently.

Although highly effective, the success of HAART depends to a large extent on how accurately patients take their medication. Soon after HAART was introduced, studies showed that the best treatment results were obtained when patients took at least $90-95 \%$ of their medication as prescribed, which is high compared to 
adherence levels considered sufficient for many other medical treatments (i.e., approximately 80\%; Howard et al., 2002; Knobel et al., 2001; Osterberg \& Blaschke, 2005; Paterson et al., 2000). Suboptimal intake of medication, from not taking doses on time to skipping (multiple) doses, leads to increased viral activity, which can result in the development of resistant viral strains and treatment failure. Although the effects differ somewhat between regimens and regimen categories, adherence levels slightly below $90 \%$ (e.g., $80-90 \%$ ) have been found to confer the greatest risk of developing drug resistant mutations, and patients taking substantially fewer doses (e.g., <75\%) experience a threefold higher chance of dying during the first year of treatment (Hogg et al., 2002; Tam et al., 2008). When HIV becomes resistant, patients usually have to switch to another regimen. However, the effectiveness of second-line treatments may also be compromised by the viral resistance, and they are generally more complex and cause more side effects. After patients experience a second treatment failure, finding a third effective and tolerable treatment is difficult and sometimes even impossible. It is therefore of major importance that patients benefit as long as possible from well-tolerated regimes that effectively suppress viral replication. Continuous high levels of adherence are vital for that purpose.

Despite that patients usually receive information about HIV, HAART and adherence at the start of the treatment, numerous studies have shown that approximately $50 \%$ of the patients take less than $95 \%$ of their medication as prescribed (Gross et al., 2006; Howard et al., 2002; Knobel et al., 2002; Knobel et al., 2001; Paterson et al., 2000). Hence, these patients are at increased risk for treatment failure, resulting in a lower quality of life and reduced life expectancy. A large number of studies has been conducted to identify the causes of non-adherence to HAART, although the use of behavioral theories in this field has been scarce (Simoni et al., 2006). Factors that have been found predictive of non-adherence can be classified as sociodemographic (e.g., age, ethnicity, gender), psychosocial (e.g., selfefficacy, forgetfulness, depression), treatment related (e.g., regimen complexity, side-effects), and environmental conditions such as patient-provider relation and access to medical care (Ammassari et al., 2002; Crespo-Fierro, 1997; Fogarty et al., 2002; Ickovics \& Meade, 2002). Building on these findings, scholars have developed a range of adherence improving interventions to enhance the efficacy of HIV health care. Some of these interventions have shown promising effects on adherence and clinical outcomes (Amico et al., 2006; Haynes et al., 2008; McDonald et al., 2002; Rueda et al., 2006; Simoni et al., 2006). However, many were non-randomized, small-scale and short-term pilot-studies, measured adherence subjectively over brief time periods (e.g., 3-day self-reports), and/or experienced considerable dropout ( $>20 \%$; Haynes et al., 2008; McDonald et al., 2002; Rueda et al., 2006). Of 


\section{CHAPTER 1}

the intervention studies without these methodological concerns, intervention programs were usually delivered by specially trained personnel who complemented patients' usual care for the duration of the study. It is therefore unlikely that the resources required for these interventions (e.g. facilities, time, and competences) are available in routine health care for HIV-infected patients.

Notwithstanding the modest number of intervention studies that report significant effects on adherence and/or viral load, and the potential for improvement in the quality of study methodology, the synthesis of these results can serve as an important source of information to guide future intervention development and clinical practice. Several systematic reviews and meta-analyses have therefore been conducted in the HAART adherence intervention field previously. While these research syntheses have shown that the published interventions do, on average, improve adherence and viral suppression compared to control conditions, it has not been possible to attribute these effects to the content of the experimental interventions (Amico et al., 2006; Rueda et al., 2006; Simoni, et al., 2006). These findings, or the lack thereof, therefore leave researchers and health care professionals with little evidence to improve their practice.

In sum, the number of HIV-infected patients receiving HAART to suppress HIV is substantial and continues to grow. Approximately half of the patients are at increased risk for treatment failure due to suboptimal medication use. Considering the absence of a firm evidence-base for adherence care, the field could benefit considerably from research that expands our understanding of effective interventions, and thereby guide clinical practice towards an evidence-based form of adherence care.

The main objective of this thesis is to contribute to the evidence-base for adherence care provided to HIV-infected patients using HAART. This is done through the systematic application of behavioral theory and scientific evidence in five studies that are described in more detail below. Although the studies focus on HAART adherence, they also address theoretical and methodological issues that may be relevant to the larger domain of behavioral science.

Since we refer to our previous work in consecutive studies, we ordered the chapters chronologically. 


\section{Overview of studies}

One line of research presented in this thesis concerns the development and testing of an intervention to enhance adherence to HAART among HIV-infected patients in the Netherlands. Data collected during two clinical trials are presented in the chapters 2, 4, and 5. First, chapter 2 describes the development and pilot-testing of the so-called Adherence Improving self-Management Study (AIMS), designed specifically to fit in HIV clinics' routine procedures. This counseling intervention was built on literature reviews of important adherence predictors and potentially effective intervention techniques. These results were integrated with theories that explain health behavior and health behavior change. Moreover, we conducted observational research during routine care to determine which adherence issues were already addressed during usual care (e.g., verbal explanation about HIV, HAART and adherence, management of side effects, basic medication planning). Finally, HIV-nurses were closely involved in the refining, complementing, and tailoring the AlMS-strategy to their perceived needs of the patients, and to the time and competences available in routine clinical care.

The main objectives of the pilot study were to evaluate whether HIV-nurses could adequately deliver the intervention during routine clinical visits, to determine whether this resulted in improved adherence among participants, and to establish whether patients and HIV-nurses evaluated the intervention positively. While we assessed adherence over time to evaluate the effectiveness of AIMS, process data were obtained through direct observation of intervention visits, and post-intervention interviews with both the HIV-nurses and the patients. These data showed that both the patients and the HIV-nurses evaluated the intervention positively, that adherence improved considerably between baseline and month three of the intervention, and that AIMS seemed to fit in routine clinical visits.

Based on these encouraging outcomes, and using the process data from the pilot to further fine-tune the intervention to routine clinical care and patient needs, we commenced a randomized controlled trial (RCT) to more rigorously evaluate the effectiveness of AIMS. This 9-month trial was run largely by the HIV-nurses who included 133 patients, managed data collection, and delivered the intervention. While in the pilot study we evaluated the effects of AIMS on adherence only, in this study we also collected viral load data to examine the possible impact of the intervention on clinical indicators of treatment success. The results of this trial are described in Chapter 4.

During the RCT, patients completed questionnaires that (amongst others) included cognitive predictors of adherence (e.g., attitude, self-efficacy, intention) as well as self-reports of so-called "preparatory behaviors and self-regulatory activities" 


\section{CHAPTER}

(e.g., carry medication along when not at home, self-monitoring of medication intake). We assessed these data to allow a detailed process evaluation of the effects of the intervention (yet to be conducted), but also to examine a theoretical idea in a clinical sample (i.e., using the control group data only). This theoretical idea was as follows: Some of the most frequently used behavioral theories consider people's intention to conduct a behavior as the most immediate predictor of that behavior in the future. However, intentions explain on average only about $30 \%$ of variance in behavior (Sheeran, 2002). Since several years scholars therefore commenced a search directed at predictors that bridge the gap between people's intentions and their behavior. The objective of this longitudinal questionnaire study, described in Chapter 5, was to contribute to this emerging field by examining whether preparatory and self-regulatory activities mediate the intention-behavior relationship.

The second line of research in this thesis exists of two meta-analyses conducted in the field of HAART adherence interventions. As reported previously, former reviews and meta-analyses have been quite unsuccessful in explaining the observed differences in intervention effects between studies. An important cause for this lack of explanatory power could lie with a methodological issue usually not addressed in meta-analyses of (behavior change) interventions, that is, variability in the quality and effectiveness of care provided to control groups in these clinical trials.

Meta-analyses of interventions typically examine the content of experimental treatments in detail, while ignoring the quality and effectiveness of care provided to control conditions (i.e., usual or standard care; we refer to this as standard care). However, as Wagner and Kanouse (2003) have also argued in a Letter to the Editor, also standard care activities may affect adherence behaviors and clinical outcomes. In addition, the quality and effectiveness of adherence care may vary between different study sites, implying that meta-analyses need to control for variability in standard care in order to accurately explain intervention effects. Since there was no theoretical framework for, nor empirical evidence of variable effectiveness in the care provided to active control groups in HAART adherence trials, the first metaanalysis we conducted focused on assessing and coding the quality of standard care, examining whether the quality of standard care varied between studies, and finally to see if standard care quality predicted treatment outcomes. This study is described in Chapter 3.

This first meta-analysis showed that the quality and effectiveness of standard care provided to control groups in HAART adherence trials varied considerably between studies. Hence, meta-analyses of intervention studies conducted in this field should assess and control for variability in care provided to the control groups to allow accurate comparisons of intervention effects. Our second meta-analyses 
(Chapter 6) was such a study, that is, its objective was to examine whether the quality of experimental interventions could accurately predict intervention effects after controlling for variability in care provided to control groups. Hence, this study was conducted with the aim to both advance understanding of effective interventions to enhance adherence to HAART through a research synthesis, but also to investigate whether indeed intervention effects are better understood after controlling for variability in care provided to control groups. 


\title{
CHAPTER 2
}

Theory- \& Evidence-based Intervention to Improve Adherence to Antiretroviral Therapy among

HIV-infected Patients in the Netherlands:

\author{
A Pilot-study
}

Published as: Marijn de Bruin, Harm J Hospers, Bart van den Borne, Gerjo Kok, \& Jan M Prins (2005). Theory- \& Evidence-based Intervention to Improve Adherence to Antiretroviral Therapy among HIV-infected Patients in the Netherlands: A Pilotstudy. AIDS Patient Care and STDs, 19(6), 384-394. 


\section{ABSTRACT}

The objectives of this study were to describe and pilot-test a theory-and evidencebased intervention to improve adherence of HIV-infected patients with antiretroviral medication. Twenty-six treatment-experienced patients (>6 months on treatment) participated in a within-subject comparison design. Adherence was measured electronically with so-called MEMS-caps for at least five months: two months before the intervention and three months during the intervention. MEMSdata were used to measure the outcome of the intervention, but also served as feedback to participants during the intervention. Mean adherence during the month before intervention was compared to mean adherence during the third month of intervention. Data for the process-evaluation were gathered through direct observation and semi-structured interviews.

Adherence improved significantly during the intervention $(Z=-2.1, p<.05)$. Mean adherence (percentage of prescribed doses taken within correct timeinterval) before the intervention was $81.8 \%$ compared to $92.5 \%$ during the third month of the intervention. More than $65 \%$ of the patients non-adherent $(<95 \%$ adherence) before the intervention were adherent during the third month of the intervention. Both healthcare professionals and patients positively evaluated the intervention-protocol and the electronic measurement of adherence. The only critique from some patients was the lack of user-friendliness of the MEMS-cap and its medication container (size and shape). It can be concluded that the proposed intervention fits in standard care procedures, can be executed by trained HIVnurses, seems to improve adherence to antiretroviral medication, and is positively evaluated by the users. Large-scale and long-term research is warranted. 


\section{INTRODUCTION}

The consequences of non-adherence for the effectiveness of medical treatments vary per disease and per medication. With respect to the treatment of HIV-infection with Highly Active Antiretroviral Therapy (HAART), non-adherence leads to inadequate suppression of viral replication at the cost of the body's immune system. In addition, frequent replication of the virus in the presence of antiretroviral medication can result in the development of resistant HIV-strains. Once a resistant strain develops, not only the current treatment loses (part of) its potency, alternative treatments may also become less effective. Furthermore, despite a large and growing number of HAART-combinations, multiple viral resistance can make it difficult or impossible to select alternative effective treatments. The general opinion is that adherence of $90 \%$ or more is required to prevent virologic failure (Howard et al., 2002; Knobel et al., 2001; McNabb et al., 2001; Paterson et al., 2000; Sethi et al., 2003). However, there is some debate on whether or not this holds under all conditions (Bangsberg et al., 2003).

Despite current attempts to optimize patients' adherence to prescribed treatments, 25 to 75 percent of the patients do not take HAART as prescribed (Bedell et al., 2000; Howard et al., 2002; Hugen et al., 2002; Liu et al., 2001; Nieuwkerk et al., 2001). The primary purpose of the present study was therefore to pilot the effect of a newly developed intervention, the Adherence Improving self-Management Strategy (AIMS), on adherence to HAART. The secondary purpose was to investigate whether or not HIV-nurses could adequately execute the intervention during standard consulting hours. Before presenting the results of the pilot-study, a behavioral model for medication adherence will be described. This model depicts the most important determinants and processes for adherence behavior, which were used to understand adherence behavior and to formulate intervention targets for behavioral change, information that is usually not included in comparable research reports (Haynes et al., 2002; Mullen et al., 1985).

\section{Understanding and changing adherence to HAART}

Based on an extensive review of behavioral theories and studies on adherencerelated factors, a behavioral model of medication adherence is presented in Figure 1. This model was mainly based on the Theory of Planned Behavior and on SelfRegulation theories, and provides insight in the factors that determine adherence behavior (Ajzen, 1991; Boekaerts et al., 2000). Based on this model, suggestions on how to change each behavioral determinant in favor of adherence to HAART are 
discussed. The strategies for changing determinants have been derived from behavioral change theories and a review of successful components of previous intervention strategies (Bartholomew et al., 2001; Bertholet et al., 2000; Epstein \& Cluss, 1982; Gollwitzer, 1993; Gollwitzer \& Oettingen, 1998; Haddad et al., 2000; Hall et al., 1988; Haynes et al., 1996; Mazzuca, 1982; Mullen et al., 1985; Peterson et al., 1984; Posovac, 1980; Posovac et al., 1985; Prochaska \& DiClemente, 1983, 1986; Rogers \& Bullman, 1995; Rollnick \& Miller, 2002; Roter et al., 1998; Stewart, 1995). The AIMS-protocol presented under methods is an integration of the described intervention strategies, structured according to the described model.

Patients gain "treatment experience" once they are exposed to a medical regimen. To enable patients to adequately self-regulate their adherence behavior, they have to be able to make informed choices about their behavior and to motivate themselves to execute the behavior correctly and at the right time. Patients must therefore possess a basic level of understanding about HIV, their antiretroviral regimen, and the short- and long-term consequences of correct and incorrect dosing and timing of intakes. Healthcare professionals must communicate this information in understandable and concrete language, tailored to the needs of each specific patient, preferably in combination with a simple illustration and written materials that patients can read at home.

A key concept in the adherence model is the intention of the patient to adhere to the medical recommendations. According to the Theory of Planned Behavior the intention to adhere to recommendations is determined by the subjective norm, the attitude and the perceived behavioral control. The subjective norm is what a person thinks other people believe (s)he should do (e.g. physicians or partners expect patients to always take medication correctly), and the motivation to comply with these normative references (e.g. high when the other is considered an appreciated, trustworthy expert). A subjective norm can be changed through either changing patients' perception of the norm, or through changing patients' motivation to comply with this norm. The attitude reflects salient beliefs about the perceived outcomes of these behaviors (e.g. adherence leads to better health than nonadherence) and the evaluations of these outcomes (e.g. good health is essential for being independent and being a good parent). It is important that patients perceive that the benefits of adhering to the treatment outweigh the costs. To optimize the attitude for adherence not only the costs of the treatment should be minimized (e.g. minimize side-effects, tailor treatment to patient's life-style, make healthcare convenient), the anticipated benefits should be maximized (e.g. link adherence to health, and health to values in life such as independence, be a good parent, feel fit, etc.). 
Perceived behavioral control (PBC) comprises two components: self-efficacy dealing largely with the perceived ease or difficulty of performing a behavior (e.g. feel confident to always take medication correctly in a private setting), and controllability (the extent to which the performance is up to the actor, e.g. due to an irregular and demanding job it is often complex to remember medication intake or have some privacy; Ajzen, 2002). Because the PBC may to some extent represent actual behavioral control and bringing a course of behavior to a successful conclusion is likely to increase with increasing $P B C$, there also exists a direct link from PBC to behavior (Ajzen, 1991). To optimize someone's PBC it is useful to separate behavior into small and simple steps (set sub-goals), facilitate the behavior where possible (e.g. use of alarm system or dose-organizer), and plan the execution of the behavior in a setting in which patients feel most confident about correctly performing the behavior on a daily basis (e.g., during breakfast at home). Discussing and planning how to execute the behaviors in more complex settings (e.g. take medication in a restaurant without anybody noticing) will probably also increase a persons' perceived and actual behavioral control.

The outcome of this process is expressed in a behavioral intention, during which patients decide what to do (e.g., accept or refuse adhering to the treatment as prescribed). When patients are ready for action, "action planning" may include defining targets (e.g., to be $100 \%$ adherent), the behaviors by which these targets should be accomplished (e.g., collect medication in time, have medication present at time of intake, consume the medication correctly), and the behavioral strategies that facilitate the correct execution of the required behavior (e.g., connect intake to a cue such as breakfast and dinner, use an alarm system or social support).

Action-planning is followed by a period action (behavior), during which patients try to execute the required behaviors adequately. During action, patients may notice in which situations or circumstances the correct execution of the behaviors is problematic or not. This self-monitored information can be used, together with feedback about changes in viral load and $\mathrm{CD} 4$, adherence measurements, side effects and relief of symptoms, during the consecutive evaluation. During this evaluation patients can use feedback to compare the actual results of their efforts with the desired results. Feedback about (non)adherence and its consequences for one's health can play a major role in behavioral change. In the first place feedback can raise awareness of current behavior and its consequences, which may motivate people to change their behavior. In the second place, by using feedback about the results of efforts to change behavior, patients can judge whether or not they have reached their targets. 
CHAPTER 2

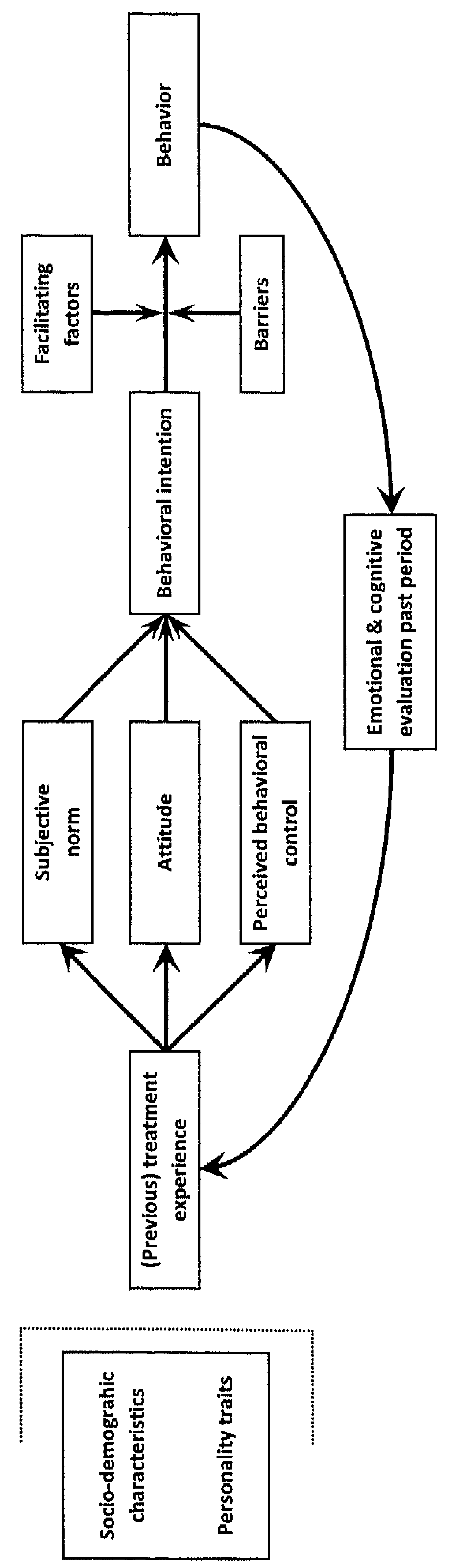

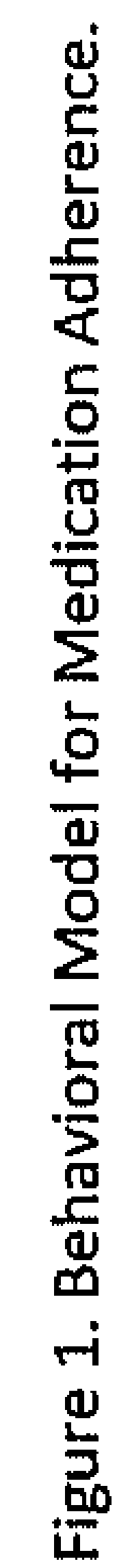


Evaluations can lead to positive or negative self-judgments. For example, if adherence to the treatment results in an increase of $\mathrm{CD} 4$ and a decrease in viral load, the efforts lead to positive emotions (or reduction of negative emotions such as fear) and an increased likelihood that the patient will perform the behavior again. When self-judgment is negative, people can either react adaptively or defensively. Adaptive reactions lead to new and better forms of self-regulation, or to choosing more effective strategies (e.g. change dosing schedule, mobilize social support, increase efforts; Zimmerman \& Martines-Pons, 1992). Defensive reactions on the other hand primarily serve to protect people from future dissatisfaction and aversive affect, which may undermine successful adaptations. Such reactions can result in a state of helplessness, procrastination, task avoidance, cognitive disengagement, and apathy; reactions that are self-handicapping and ultimately limit personal growth (e.g. deny non-adherence, blame non-adherence on a uncontrolllable factor, express belief that adherence is not that important; Garcia \& Pintrich, 1994). In case of negative self-judgments, adaptive reactions and learning from the past is of major importance for future motivation and the improvement of adherence-efforts. Healthcare professionals and people providing social support can help patients to react adaptively. A good (patient-healthcare professional) relationship, good communication skills and communication techniques such as those used in Motivational Interviewing are essential in this respect (Rollnick \& Miller, 2002).

The outcomes of the evaluation increase patients' treatment experience and can result in feelings of either satisfaction that can strengthen self-efficacy beliefs and intrinsic interest in the task, or self-dissatisfaction that can lead to reduced senses of self-efficacy and to reduced intrinsic interest in pursuing the target behavior(s) any further (Schunk, 1996; Zimmerman \& Kitsantas, 1996). Increased treatment experience is also likely to result in a change of attitude as the patient has experienced the actual pros and the cons of the treatment. Finally, as patients' experience with the treatment increases, they may perceive themselves as the most appreciated, trustworthy expert and be less inclined to comply with the perceived norms of other people. Thus, new treatment experiences can influence the subjecttive norm, the attitude and perceived behavioral control, and therefore patients' intentions for the upcoming period. These same experiences can be used in a practical manner to further tailor the treatment to the life-style of the patient (e.g. change time of intake, change dosing frequency, use electronic reminders).

After processing the new treatment experiences, again patients enter the action- and self-monitoring phase, followed by an evaluation, an increase of treatment experience, self-reactions that may influence motivation, and so forth. In time the behavior, if performed frequently and in the same contexts, may become habitual so that conscious decision making and deliberation is no longer required 


\section{CHAPTER 2}

(Ouellette \& Wood, 1998). However, this equilibrium can be disturbed by a change in factors such as new side effects, loss of social support, changes that disturb the daily rhythm, or because other important goals, such as personal relationships or financial problems, conflict with the behaviors conditional for adherence. If the balance is disturbed, patients will again have to go through the cycle of motivation, action-planning, action, evaluation and self-reactions to find a new balance. For patients who do not have a regular life-style so that the intake of medication can not become habitual, the intake of medication remains a conscious effort that requires planning and the use of alternative cues to remember the intake of medication.

\section{MATERIALS AND METHODS}

So-called MEMS-caps (AARDEX Ltd, Switzerland) were used to measure patients' medication intake. MEMS-caps are electronic caps that fit on standard pill-bottles. They register date and time of every opening of the pill-bottle, and are considered as the best measurement instrument currently available (Bertholet et al., 2000; Bond \& Hussar, 1991; Kastrissios \& Blaschke, 1997; Liu et al., 2001; Urquhart, 1997). The data can be downloaded from the MEMS-cap with a communication device and a computer, and Powerview software (AARDEX Ltd.) presents the data in simple plots. These plots can be used to provide feedback about behavior, and to identify suboptimal adherence-patterns, both essential components of the AIM Strategy. Also, patients were offered another type of MEMS-cap during the intervention: the so-called MEMS-view cap (or SmartCap). Besides recording adherence like a regular MEMS-cap, this cap displays how often it has been opened during that day and how many hours have passed since the bottle was last opened. This cap can help patients to check whether or not the medicines have already been consumed, and to increase awareness when doses are missed.

\section{The intervention}

At each patient visit, a trained HIV-nurse downloaded the data from the MEMS-cap and judged patients' adherence. If adherence was adequate, feedback was provided with a printed version of the results and the patient was reinforced. If adherence was suboptimal the intervention protocol was initiated. This protocol consisted of the following nine steps: 1] The HIV-nurse starts with providing practical, tailored 
knowledge about adherence (verbally and using simple graphs). 2] The HIV-nurse asks the patient to define a desired status using exemplar reports varying from excellent adherence to poor adherence. If an insufficient level of adherence is selected, the reasons for and consequences of this choice are discussed with the aim to improve patient's desired status. 3] The adherence-data (actual status) is printed and discussed with the patient (feedback). A difference between the actual and desired status is the source for motivation to change, and the HIV-nurse stimulates the patient to react adaptively. 4] The HIV-nurse and the patient define the causes of non-adherence (lack of understanding, lack of motivation, insufficient action plan, barriers). The detailed MEMS-reports serve as the starting point for this discussion. 5] The HIV nurse and the patient formulate solutions to solve or avoid the causes for non-adherence. These solutions are formulated as implementation intentions in an action plan (Gollwitzer \& Oettingen, 1998). 6] Patients take home their adherence results, action plan and a SmartCap, and are asked to self-monitor their behavior and the circumstances in which they experience problems consuming the medication correctly and on time. They are also asked to autonomously develop and implement solutions to cope with these problems in the future. 7] When the patient returns for consecutive intervention sessions (time between two sessions depends on the degree of non-adherence), adherence is evaluated, problems and solutions discussed, action plans adapted, until the patient demonstrates a sufficient level of adherence. 8] Once adherence is sufficient, goals for maintenance of adherence are formulated. 9] After the patient has demonstrated sufficient levels of adherence for two or three months, the new behaviors are considered stable and the intervention a success.

Participants who take $\geq 95 \%$ of their medication on time are considered adherent. However, the target of the intervention is to aim for maximum levels of adherence for all patients.

\section{Design}

The intervention was pilot-tested in a within-subject comparison design with a preintervention measurement of adherence of at least two months and an intervention period of at least three months. The study was performed at the HIV outpatient clinic of the Academic Medical Centre (AMC) in Amsterdam, the Netherlands. Approval for the study was obtained from the AMC-Medical Ethical Committee and all patients gave informed consent to participate in the study. 


\section{Participants}

Twenty-six patients were included in the pilot-study. Inclusion criteria were treatment experience ( $>6$ months on HAART), Dutch speaking, a western cultural background, age 18 years and older, and no known psychiatric problems or alcohol and drug abuse. Patients were enrolled from January to March 2003.

\section{Measures}

Adherence measurements with MEMS-caps permit the use of a specific primary outcome measure, so-called timing compliance: the percentage of doses that has been consumed within a certain time-interval. The correct time-interval was set on 10-14 hours if the drug had to be taken twice daily, and 20-28 hours for once-daily regimens. Two secondary outcome measures were taking compliance (the percentage of doses taken) and dosing compliance (the percentage of days with correct amount of intakes; Vrijens \& Goetghebeur, 1997). These secondary measures are more widely used, but also more insensitive (more forgiving) to errors in dosing and timing.

Process evaluations were performed to 1. observe whether or not the AIMSprotocol could effectively help patients solve adherence-related problems, and 2 . to determine patients' and HIV-nurses' satisfaction with the AIM-Strategy. The data for the process evaluation were gathered through direct observation and participation during the intervention sessions, as well as through semi-structured, open-ended interviews with a subset of 13 patients and all the HIV-nurses. Those thirteen patients were asked to score three aspects of the intervention on a scale from 1-10, with 1 as very bad/not useful at all, and 10 as excellent/extremely useful. Patient characteristics were obtained from a hospital database provided by the HIV Monitoring Foundation, the Netherlands, and from patients' files at the hospital.

\section{Analyses}

Mean timing, taking and dosing compliance were calculated per patient per month using the Powerview software. Adherence data from the month before the intervention was compared to adherence data during the third month of the intervention. Because the adherence data were nonparametric, the Wilcoxon Matched-Pairs Signed-Ranks test was used to test for significant differences in adherence over time. All the other data are descriptive. 


\section{RESULTS}

Twenty-six patients were included in the AIMS-pilot study. An unfortunate loss of data was found among four patients who used medication organizers (one week's medication organized in a box) and who were incorrectly instructed about how to use the MEMS-caps for the registration of their medication intake. One other patient died, and one patient dropped out the study because of private reasons. One patient did not want to continue using the MEMS-cap after two weeks and two patients dropped out of the study just before completing the intervention period. However, the data from the latter two patients were sufficient to be included in the analysis, resulting in a total of nineteen patients with adequate data.

All participants were Dutch, 18 males and 1 female, with a mean age of 48.2 years (SD 11.5). Fifteen participants used twice-daily regimes, 3 patients took medication once a day, and 1 patient took medication three times per day. One patient used antiretroviral medication for 5 months, three for 6-24 months, three for 2-5 years, and twelve for more than 5 years. All but two patients had an undetectable plasma HIV-1 RNA ( $<50$ copies/ml). One patient had a CD4 count below 200, eight patients had a CD4 count between 201-500, and ten participants had a CD4 count of over $500 \times 106 /$ ( (see Table 1).

\section{Adherence to HAART before and after intervention}

At baseline, $52.6 \%$ of the participants were non-adherent $(<95 \%$ timing compliance). This percentage decreased with increasing forgiveness of intake-errors on the secondary measures: $31.6 \%$ were not adherent in terms of dosing compliance, while $10.5 \%$ were non-adherent in terms of taking compliance. During the third month of the intervention, $15.8 \%$ of the patients were non-adherent in terms of timing compliance, $10.5 \%$ in terms of dosing compliance, and all patients took $95-$ $100 \%$ of the medication. Patients received a mean of 1.7 intervention sessions, varying from 10 to 45 minutes depending on the complexity of the problems.

Adherence significantly increased in all outcome measures: timing compliance $(Z=-2.1, p<.05)$, dosing compliance $(Z=-2.8, P<.01)$, and taking compliance $(Z=-$ $2.1, p<.05)$. Figure 2 shows the mean adherence per measure at baseline and during the third month of the intervention. 


\section{CHAPTER 2}

\section{Process evaluation of the AIM-Strategy}

The majority of patients actively participated in the process of problem identification and problem solution. In general, patients successfully used the solutions and the materials (e.g., SmartCap, alarm devices) to change their behavior if needed. The most frequently observed causes for non-adherence were lack of knowledge about adherence, not knowing when medication is taken correctly, unawareness of non-adherent behavior, social pressure, social situations in which patients did not want to take the medication, negative feelings about the importance of medication in daily life, belief that $95 \%$ adherence was in their case not necessary, forgetfulness, and inadequate action plans. The most frequently applied adherence improving strategies were: increasing patients' understanding of the importance of adherence, providing specific information about when the antiretroviral combination is taken correctly, using MEMS-reports to raise awareness about current behavior and to provide feedback about behavior change, relieving social pressure or its effect on the patient, and adapting the action plan so that a variety of problems could be avoided, solved, or prevented.

The evaluation regarding patients' and nurses' satisfaction with the AIMStrategy pertained both to the materials used (MEMS-caps, software, reports) as well as to the intervention protocol. Many patients reported that MEMS-caps were not as user-friendly as the medication containers they normally used, due to their size, color, and the rattling sound of the medicines. For those reasons, one patient dropped out of the study after two weeks, while two others did not complete the whole intervention period. Participants were more positive about the extra possibilities of the SmartCap, in that it was helpful to confirm whether or not they had already taken their medicines that day. At the end of the study, when most people used a SmartCap, patients were asked to score the user-friendliness of the MEMScaps on a scale from 1 (low)-10 (high). The mean score was 7.2 (SD 1.6).

Participants were positive about the manner in which the data were presented in the adherence reports. Most participants rapidly mastered reading the reports independently from the HIV-nurse, used the reports to look for patterns of suboptimal adherence, formulated targets for the next meeting, and took the reports with them for personal dossiers or to show it to relatives. When asked to evaluate the utility of the reports on a scale from 1-10, the mean score was 8.4 (SD 0.9). 
Table 1: Characteristics of the study subjects $(n=19)$

\begin{tabular}{llc}
\hline Mean age & Years (standard deviation) & 48.21 (11.51) \\
Ethnicity & Dutch & 19 \\
Gender & Male & 18 \\
& Female & 1 \\
Sexual orientation & Heterosexual & 5 \\
& Homosexual & 14 \\
Doses per day & One & 3 \\
& Two & 15 \\
Duration of antiretroviral use & Three & 1 \\
& $\leq 6$ months & $1(5$ months) \\
& $6-24$ months & 3 \\
Plasma HIV-1 RNA (copies/mi) & $2-5$ years & 3 \\
& $>5$ years & 12 \\
& $<50$ & 17 \\
CD4 + T-lymphocyte count (cells/ $\mu$ l) & $50-10.000$ & 0 \\
& $>10.000$ & 2 \\
& $\leq 200$ & 1 \\
& $201-500$ & 8 \\
& $>500$ & 10 \\
\hline
\end{tabular}

Patients evaluated the AIM-Strategy positively. Examples of comments were "I learned nothing new, but it is nice to demonstrate that 1 am as adherent as 1 thought I was" and "I feel like I am allowed to be a patient again and can become 90 years old now". When asked to score the content of the program on a scale of 1-10, the mean score for the program was 8.2 (SD 0.7).

The HIV-nurses, although expressing the need for a user-friendlier version of Powerview, also evaluated the AIM-Strategy positively. The general opinion was that the MEMS-cap provided an excellent insight in the behavior of patients, that the reports were a good basis for intervention, the educational materials were helpful, and the protocol provided a practical structure for intervening with nonadherence. However, the adequate execution of all different components of the intervention within a 30-minute consultation was difficult for some nurses. Nevertheless, the HIV-nurses expressed the confidence that additional practice would provide them with sufficient skills to provide the AIM-Strategy properly within the available time. All HIV-nurses expressed the desire to continue with AIMS-counseling. 


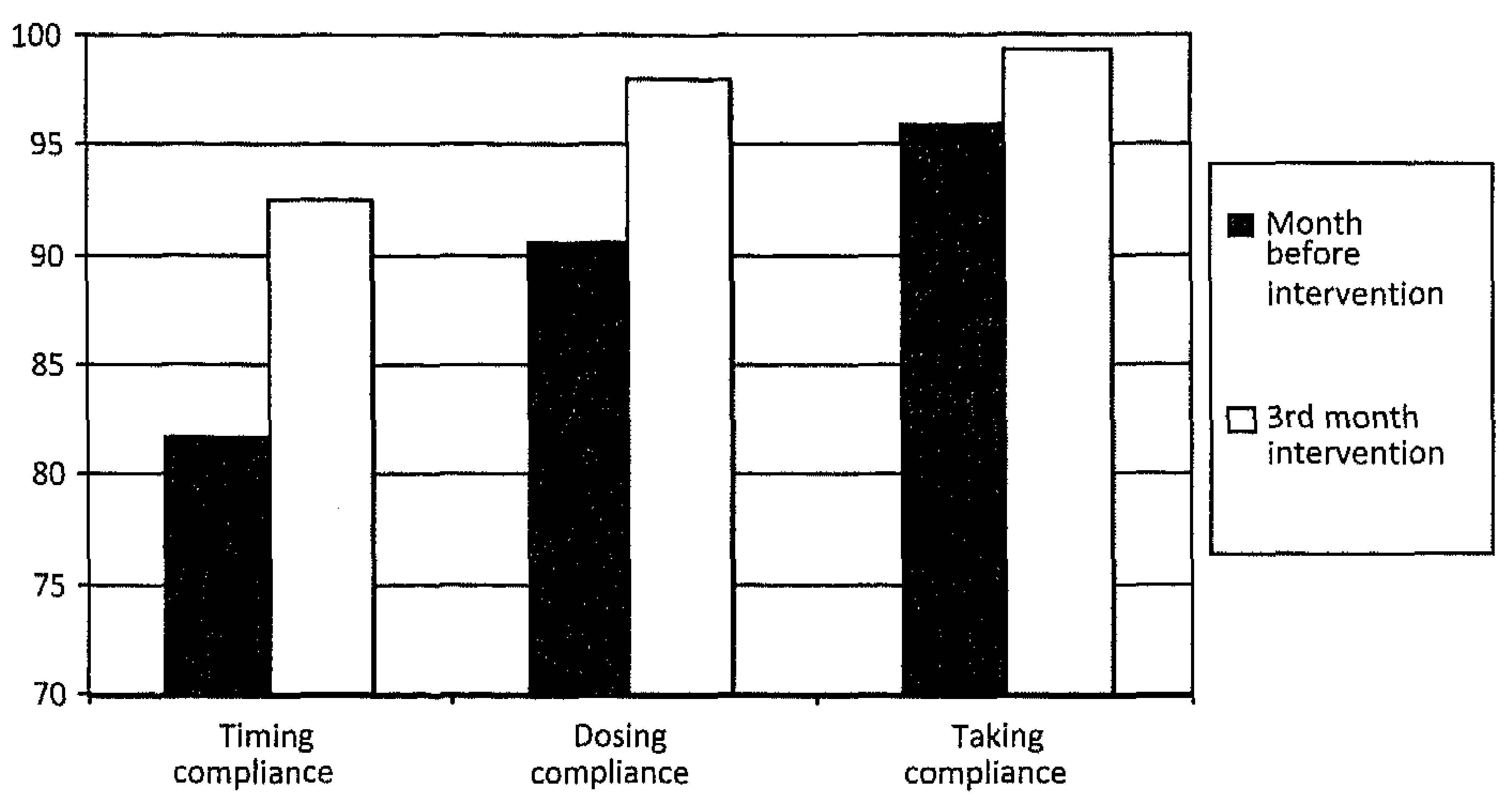

Figure 2: Mean timing, dosing and taking compliance at baseline and during third month of intervention

\section{DISCUSSION}

The aim of this pilot-study was to investigate whether or not adherence to HAART among HIV-infected patients could be improved with the AIM-Strategy. At baseline, adherence was insufficient among fifty-three percent of the patients. During the third month of the intervention, sixteen percent ( 3 patients) remained nonadherent in terms of the primary outcome measure timing compliance. Nevertheless, two out of these three non-adherent patients substantially improved on dosing and taking compliance. The overall improvements in timing, dosing and taking compliance between baseline and the third month of the intervention were significant.

The factors that were related to non-adherence differed from a lack of knowledge and understanding, to a lack of motivation, inappropriate action plans, and barriers to the proper execution of the planned behaviors in daily life. These factors corresponded with the proposed theoretical framework. During the study it also became clear that these factors were often interrelated. Hence, interventions to improve adherence should include a complete and detailed analysis of each patient's individual situation before focusing on solutions. 
At the start of the intervention many patients did not understand the principles underlying adherence, and action plans of many patients did not fit with their daily lives. According to self-regulatory principles used in the AIM-Strategy, understandding of the principles behind adherence to a regimen is necessary for people to make informed decisions about their behavior and to manage their lives with HAART adequately. This lack of self-management is also represented by the lack of tailored action plans, as only patients themselves can notice their problems and initiate efforts to change their action plans. In other words, it seemed that most non-adherent patients lacked vital knowledge and skills for optimal self-management of their lives with HAART.

The AIM-Strategy focused on improving patients' self-management capacities. As a result of the AlM-Strategy, most non-adherent patients improved their understanding of the importance of adherence for their goals in future life, became motivated to change behavior, redefined action plans, increased self monitoring of behavior, generated and implemented solutions for problems, and successfully changed behavior. Despite the fact that these changes were only measured in a three-month period, it seems that the AIM-Strategy increases HIV-infected patients' self-management capacities and adherence to HAART. Whether or not the increased self-management competencies will remain and result in similar or higher levels of adherence on the long term has yet to be investigated.

All participants reacted positively to every aspect of the intervention except to the MEMS-caps. While only one patient expressed to have the unpleasant sensation of being "constantly observed", a few more said that the MEMS-caps was not very user-friendly compared to the medication containers they normally used. This seemed especially to be a problem for patients who regularly took their medication in a public environment and did not want other people to know that they were using medication. However, only a few patients expressed the desire to prematurely stop using the MEMS-cap. It is nevertheless desirable to have electronic monitoring devices that are smaller, look less like medication containers, and have more compartments. This may also increase the likelihood of its correct use in long-term HAART-adherence studies, especially when the MEMS is used for the observation of adherence only.

Another target of the intervention was to investigate whether or not the AIMStrategy could be adequately applied by HIV-nurses in standard care. Although some practice with and adaptations of the intervention were necessary, it now seems that the AIMS-protocol has become an understandable protocol that can be adequately executed by HIV-nurses. Increasing experience and additional communication training is expected to further increase the quality of AIMS-counseling and reduce the required time per patient. 
Several limitations of this pilot-study warrant discussion. First, it should be noted that only patients who were willing to change their behavior may have agreed to participate in this pilot-study and that during most intervention sessions the researcher either observed or participated in the intervention sessions. Second, the number of participants was limited and the results should therefore be considered as an indication of the potential effect of AIMS-counseling on patients' adherence to HAART. Finally, the effects of AIMS-counseling on adherence in this pilot-study have been short-term and derived from a homogeneous group of people. Therefore, further research should also focus on non-adherence among other target groups, such as patient who start HAART, including an inventory of the reasons and solutions for non-adherence among these different patients groups. Finally, the cost-effectiveness of AIMS and the long-term effect of AIMS-counseling on adherence and physical outcomes should be investigated.

\section{Conclusion}

The results obtained in this pilot-study suggest that large-scale and long-term research with the Adherence Improving self-Management Strategy is useful. In the first place, despite the selection of stable and virus-undetectable subjects, nonadherence was a significant problem. In the second place, the Adherence Improving self-Management Strategy seems to have the potential to be used by trained HIVnurses during consulting hours and enable them to adequately measure and improve adherence to HAART. Finally, the users of the intervention, HIV-nurses and patients, evaluated the AIM-Strategy positively. The lessons learned in this pilotstudy should facilitate a successful large-scale implementation of AIMS. However, depending on the organization and the background of the HIV-nurses, it may take some time and effort to properly train HIV-nurses to adequately execute the intervention within the available time, to integrate AIMS-counseling in existing organizational procedures, and to adapt AIMS to the demands of the specific treatment structure and patient group. 


\section{CHAPTER 3}

\section{Variability in Standard Care Quality of HAART-adherence Studies: \\ Implications for the Interpretation and Comparison of Intervention Effects}

Accepted for publication as: Marijn de Bruin, Wolfgang Viechtbauer, Harm J Hospers, Herman P Schaalma, Gerjo Kok (2009). Variability in standard care quality of HAART-adherence studies: Implications for the interpretation and comparison of intervention effects. Health Psychology. 


\section{CHAPTER 3}

\section{ABSTRACT}

Background: Clinical trials of behavioral interventions seek to enhance evidencebased health care. However, in case the quality of standard care provided to control conditions varies between studies and affects outcomes, intervention effects cannot be directly interpreted, compared, nor generalized to other settings.

Objective: To examine if standard care quality ( $S C Q$ ) could be reliably assessed, varies between studies of HAART-adherence interventions, and is related to the proportion of patients achieving an undetectable viral load ("success rate").

Design: Databases were searched for relevant articles. Authors of selected studies retrospectively completed a checklist with standard care activities, which were coded to compute SCQ scores. The relationship between SCQ and the success rates was examined using meta-regression.

Results: Reliability of the SCQ instrument was high (Cronbach's $\alpha=.91$ ). SCQ scores ranged from 3.7-27.8 (total range 0-30) and were highly predictive of the success rate $(P=.002)$.

Conclusions: Variation in SCQ provided to control groups may substantially influence effect sizes of behavior change interventions. Future trials should therefore assess and report SCQ, and meta-analyses should control for variability in SCQ, thereby producing more accurate estimates of the effectiveness of behavior change interventions. 


\section{INTRODUCTION}

Since non-adherence to medical advice is a common problem, behavioral intervenetions can make an important contribution to evidence-based health care. Numerous studies have been conducted to test the effectiveness of interventions to improve adherence to medical treatments (Haynes et al., 2005; Ismail et al., 2004; McDonald et al., 2002; Simoni, et al., 2006). Systematic reviews and meta-analyses of these studies should allow health care professionals to make rational decisions about evidence-based care using the entirety of available evidence (Higgins \& Green, 2005). However, whereas intervention characteristics are usually described and analyzed in detail in research trials and meta-analyses, the quality of care provided to control conditions receives considerably less attention (i.e., what is normally described as "standard" or "usual" care. In this article we refer to it as "standard care"). Yet, as Wagner and Kanouse (2003) have argued, also standard care activities may affect adherence behaviors and thus clinical outcomes. In addition, standard care practices may vary between different sites, implying that the assessment and reporting of standard care is essential for the interpretation and compareson of intervention effects. To investigate this alarming idea, we focused on studies that evaluate the effectiveness of interventions to improve adherence to HAART. Our aim was to develop a tool to reliably assess standard care quality, determine if standard care quality varied between studies, and examine whether standard care quality was related to the proportion of patients achieving undetectable viral loads ("success rate") while receiving standard care only.

Theory- and evidence-based behavior change interventions to promote health behaviors consist of a set of techniques (e.g., education, feedback, goal setting), intended to change specific determinants of behaviors (e.g., education to increase knowledge, feedback to raise awareness, goal setting to enhance self-efficacy). The determinants targeted by a systematically developed intervention are those that have been identified as most important for the target behaviors. Both identifying the important determinants and selecting corresponding behavior change techniques are based on behavioral theory and scientific evidence. Theoretically, the adequate application of relevant behavior change techniques in practical intervention activities should alter the most important behavioral determinants, which in turn should change behaviors, ultimately leading to improved health and quality of life of those targeted by the intervention (Bartholomew et al., 2006). Based upon this framework, the quality of an intervention can be defined as the degree to which effective behavior change techniques are adequately applied to important determinants of target behaviors. 
Once a behavior change intervention has been developed, its efficacy is usually investigated in a trial comparing an intervention condition with a control condition. In general the control condition is, just as the intervention condition, exposed to behavior change techniques. For example during standard clinical care that may encompass techniques such as education to increase knowledge about the disease and treatment, persuasive communication about the benefits of adherence, or planning coping responses to deal with anticipated problems. Just as in the intervention group, these techniques have the potential to alter important determinants and consequently behavior and health. Therefore, testing the effectiveness of a behavior change intervention in a clinical trial is actually a test of the hypothesized superior quality of one intervention ("intervention care") over another ("standard care"). Since the potential superiority of intervention care depends on the opportunity to alter important determinants of behavior that have not been (adequately) addressed by standard care, the quality level of standard care determines to a large extent how much improvement in determinants, behavior and health can be achieved with a behavior change intervention.

In meta-analyses of behavior change interventions, study outcomes are first converted into a common effect size measure, which enables comparison of study effects. If standard care quality affects health behavior and consequently clinical outcomes, the effect size produced by a similar intervention in settings with different standard care quality will be different (just as interventions of different quality tested against standard care with different quality can result in a similar effect size). Then, if the quality of standard care varies between studies, intervention effects cannot be interpreted without ample descriptions of the level of standard care against which these effects were achieved. In addition, meta-analyses not controlling for variability in standard care quality may then under- or overestimate overall intervention quality and the effectiveness of intervention techniques.

\section{Study objectives}

Our first aim was to use the theoretical framework for intervention quality, as described in the introduction, to develop a tool to assess standard care quality. This assessment tool was then used to determine standard care quality in studies that investigate the effectiveness of interventions to improve adherence to HAART. Finally, we examined whether standard care quality was actually associated with clinical success rates among HIV-infected patients who receive standard care, and whether variations in standard care quality explained clinically relevant differences in success rates between the studies. 


\section{METHODS}

Literature search and selection procedure

Embase, PsycINFO, and Medline were searched for articles describing HAART adherence intervention trials published between January 1996 and December 2006. The included search terms concerned the disease or treatment (HIV, AIDS, HAART, antiretroviral, ARV), the behavior [(non)adherence, (non)compliance, (non)persistence)], and the intervention (interv*, improv*, promot*, increas*, enhanc*) in the title or abstract. In total 4603 records were examined (including 1527 duplicates). The aim was to identify adherence intervention studies with a within- or betweensubject design. Target groups operating in a fundamentally different context, or who face challenges that are essentially different from those of the main study sample, were excluded: intravenous drug users, age $<18$ years, studies conducted in developing countries, alcohol dependent people or people with psychiatric problems. In addition to this search, published reviews on HAART adherence improving interventions were examined to check for additional studies; however, no new articles were found. Of the examined records, 47 appeared to address intervention effectiveness trials related to HAART adherence and were examined in more detail. Two reviewers (authors $M d B$ and $H J H$ ) decided which of these were relevant. Eighteen studies were excluded due to methodological inferiority (historic control group, biased control condition), no intervention aimed at adherence, or DOT-intervention (directly observed therapy, no intervention on autonomous behavior). This yielded a final sample of 29 studies that were included in the present review.

\section{Assessment of standard care quality}

We developed a checklist presenting a wide range of practical adherence activities for the assessment of standard care (see Appendix). These activities were derived from descriptions of standard care activities in the 29 selected studies, combined with observations during participatory research in the HIV-clinic of the Academic Medical Centre, Amsterdam, the Netherlands. Next, this checklist was sent to the corresponding authors of the selected studies. These authors were asked whether or not activities had been "delivered systematically to all patients" in their standard care condition. Authors responded by checking "Yes", "No", or a "?" (Don't know) and were prompted to report additional standard adherence care activities that were not listed. Items in the standard care checklist that were answered with a question mark were recoded to "No". 


\section{CHAPTER 3}

To determine which standard care activities could be considered as behavior change techniques targeting important determinants of adherence behaviors, we developed a coding manual including a taxonomy of behavior change techniques (available from the authors). This 41-item taxonomy is an adapted version of the 26item taxonomy developed by Abraham and Michie (2008). All the techniques described in the adapted taxonomy are organized under the determinant they are supposed to affect. These determinants are derived from an integration of theoretical constructs from prevailing behavior (change) theories that have been found predictive of a range of different health behaviors (Bartholomew et al., 2006). Two coders, both social psychologists with extensive experience in developing theory- and evidence-based behavior change interventions (authors GK and HPS), were trained to use the taxonomy. The coders independently coded the complete range of standard care activities in the checklist (i.e. including the additional activities reported by individual authors) and discussed their findings. Differences in coding (i.e., three instances) were resolved through discussion. The coders identified a total of 27 techniques in the standard care checklist (see Table 1). This coding was then applied to code all individual checklists completed by the selected authors.

\section{Scoring procedure for standard care quality}

Since the techniques in the taxonomy were all considered effective and targeting important determinants of adherence, all techniques in the standard care checklist were assumed to contribute equally to the score of standard care quality and received one point each. However, 7 of the 27 techniques concern long-term activities which may recur during consecutive consultations. Therefore, these techniques were given two points. Note that three techniques comprise multiple standard care checklist items, which could result in partial scores (e.g., if 3 out of 5 educational items in the standard care checklist were answered with "Yes", the score for the technique Provide general information would be 0.6 ). The theoretical range of standard care quality scores was 0-34 points.

\section{Data assessment for the meta-analysis}

Half the studies focused on HAART-experienced patients continuing their current treatment, the other half on patients starting a new treatment (new patients and 
patients switching treatment). The success rates for these two types of studies were determined as follows. To allow treatment naive patients commencing treatment to achieve an undetectable viral load, we collected the proportion of patients with an undetectable viral load in the control group after six months of standard care. Since treatment-experienced patients were already exposed to standard care at baseline, and for several practical and statistical reasons ${ }^{1}$, we used baseline data from these studies.

Standard care quality was used as the main predictor in a mixed-effects metaregression model, using either the raw or the logit transformed success rates as the outcome measure. Since the analyses for both outcome measures yielded essentially identical results, only the results for raw proportions are presented below. The amount of (residual) heterogeneity was estimated via restricted maximum likelihood. Based on methodological and theoretical considerations, we decided a priori to control for four potential moderators: 1 . Inclusion of patients continuing treatment vs. patients starting (a new) treatment, 2. Dominant ethnicity of the study sample (Caucasian vs. Other, i.e. African-American or Hispanic; Golin et al., 2002; Nellen et al., 2004), 3. Baseline selection of patients with adherence problems vs. no selection (Amico et al., 2006), and 4. Sensitivity of viral load assessments ( $4000 \mathrm{vs.} \geq$ 400 copies $/ \mathrm{mL}$ ). Standard care quality plus the other four moderators were simultaneously included in the meta-regression model. We additionally examined (separately and in combination with the other moderators) whether the collection year of the viral load data was a relevant moderator (as regimen complexity has decreased over the years, and it efficacy has increased). Two coders (authors MdB, WV) independently examined the articles for success rate data and moderators. If the necessary data was not reported in an article, the authors were asked to provide these additional data.

\footnotetext{
${ }^{1}$ We used baseline data from these studies because these patients received standard care prior to the baseline assessment. The baseline data therefore reflect the success rate under standard care conditions. The use of baseline data also allowed us to include all the participants in the analysis. Moreover, potential bias due to differential dropout across the studies could be avoided this way. Finally, it allowed standardization of the assessment time point. To control for the fact that we used baseline data for these studies and 6-month data for the other set of studies, we included a dummy-coded moderator variable in the model.
} 
CHAPTER 3

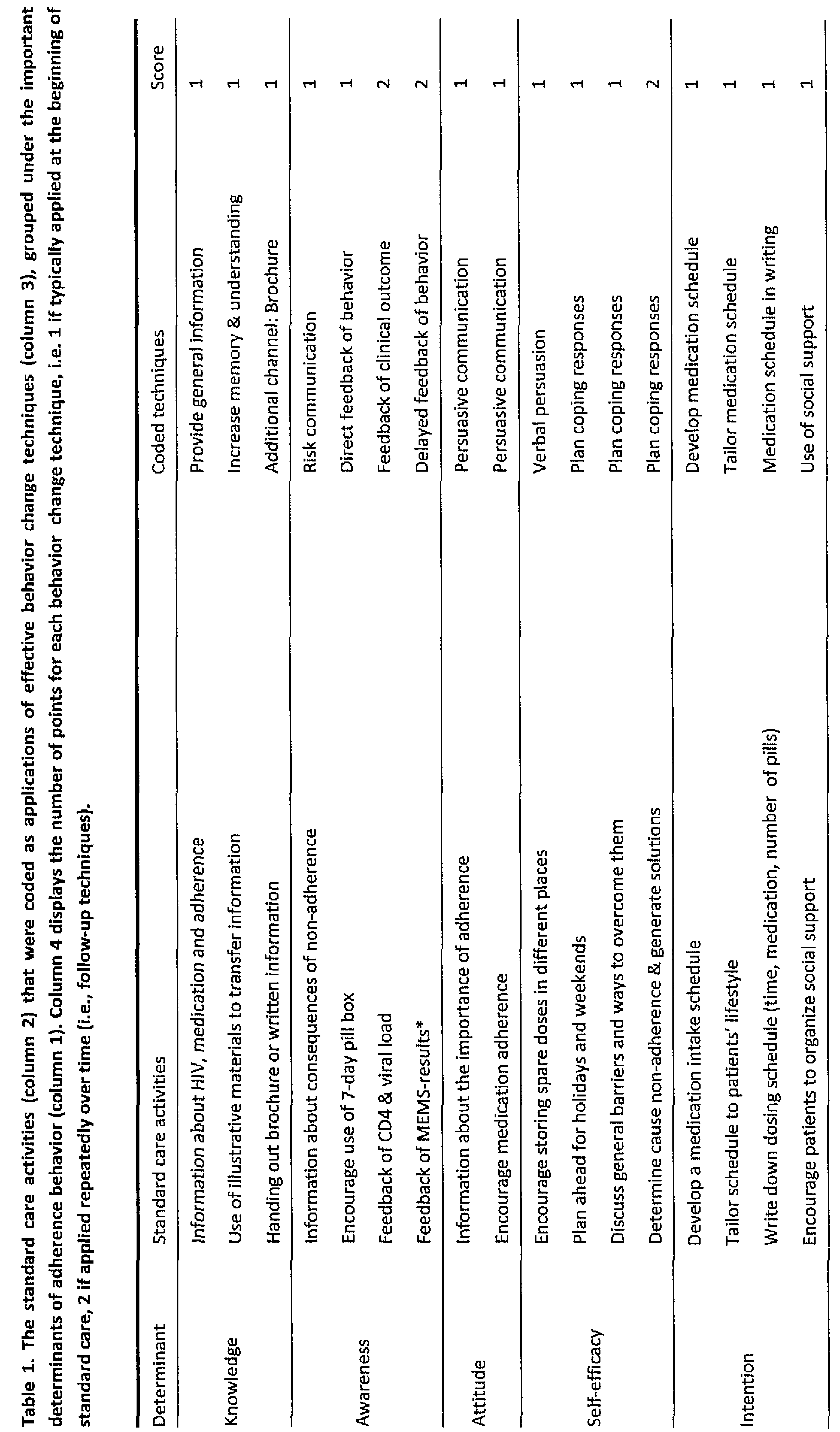


Standard care matters

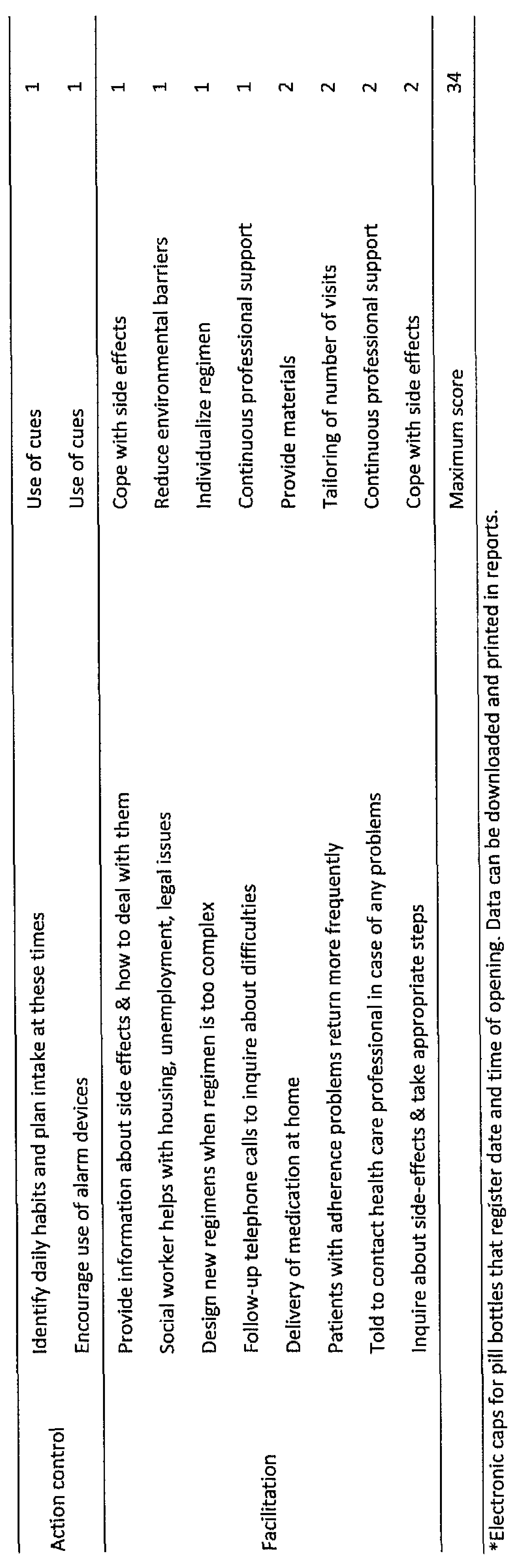




\section{CHAPTER 3}

\section{RESULTS}

Of the 29 articles selected for this study, 27 authors responded to the request for completing the standard care checklist. The two remaining authors could not be contacted. Of the responding authors, nine could not provide sufficient information about the standard care that was delivered to the patients in their study. The remaining authors responded by completing the standard care checklist, with only occasional missing data or "Unknowns" (mean (sd) 1.8 (2.0), range [0-5] out of 27 techniques (Andrade et al., 2005; de Bruin et al., 2005; Dilorio et al., 2003; Fairley et al., 2003; Goujard et al., 2003; Holzemer et al., 2006; Knobel et al., 1999; Levy et al., 2004; McPherson-Baker et al., 2000; Murphy et al., 2002; Pradier et al., 2003; Rathbun et al., 2005; Remien et al., 2005; Tuldra et al., 2000; Van Servellen et al., 2005; Wagner et al., 2006; Weber et al., 2004; Wohl et al., 2006).

We computed the quality scores per study for each determinant in Table 1 . For the three determinants that are also relevant for follow-up visits (the 2-point techniques under awareness, self-efficacy, and facilitation in Table 1), the follow-up scores were included separately. We computed Cronbach's $\alpha$ to check the internal consistency reliability of the standard care quality instrument. When the mean scores of all subscales (i.e., determinants) were included, Cronbach's $\alpha$ was .895 . Omitting the subscale Follow-up Awareness led to a relevant increase in Cronbach's $\alpha$ to .909. The high internal consistency is also expressed in the corrected item-total correlations between all subscales (range $r=.52-.90$, all $p<.05$ ) - except Follow-up Awareness $(r=.01, n . s$.) - with the total score. Based upon these analyses, the subscale Follow-up Awareness was excluded from the final scale. The final scores ranged from 3.7 to 27.8 (total range $0-30$ ) with a mean of $15.5(\mathrm{sd}=8.0$ ) and a median of 17.0 (see Figure 1).

Of the 18 studies, 14 measured and reported viral loads. However, one study selected only treatment experienced patients with undetectable viral loads (100\% success) making it unsuitable for inclusion in the meta-analysis. Table 2 contains the data for the studies included in the meta-analysis. Data on the viral load status for a total of 1516 patients was available from these 13 studies. The average proportion of patients with an undetectable viral load was estimated to be .48 ( $95 \%$ confidence interval $[\mathrm{Cl}], .39$ to $.57 ; \mathrm{P}<.001)$ in an initial random-effects model without moderators. However, the results were clearly heterogeneous $(Q=135.51, \mathrm{df}=12, \mathrm{P}<.001$; $12=92 \%$ ). Approximately $70 \%$ of the total amount of heterogeneity could be accounted for based on standard care quality and the other four moderators. The set of moderators as a whole was significant $(P<.001)$. Of the individual moderators, standard care quality and ethnicity were significant $(P=.002$ and $P=$ 
.006 , respectively; all other $\mathrm{P} \geq .19$; removing the three non-significant moderators from the model did not alter the conclusions). Moreover, collection year of the viral load data was not a significant moderator when examined by itself or in combination with the other moderators $(P=.66$ and $P=.29$, respectively).

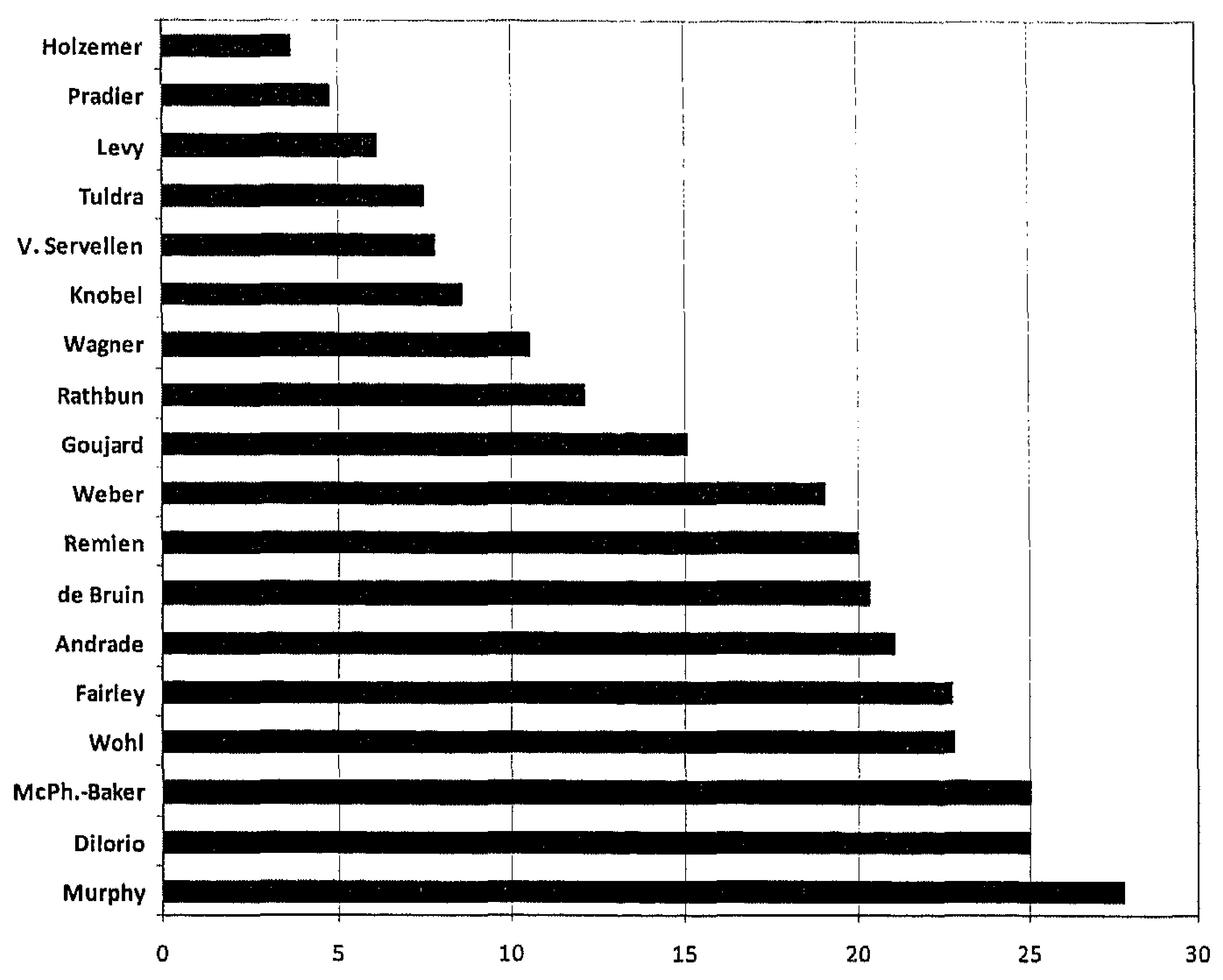

Figure 1. Variability in standard care quality

Most notably, for a one-point increase in standard care quality, the proportion of patients with an undetectable viral load was estimated to increase on average by .014 points $(95 \% \mathrm{Cl}, 0.005$ to 0.023$)$. Therefore, a 15 point difference in standard care quality (the interquartile range for this variable) corresponds, on average, to a $21 \%$ difference in the proportion of patients with an undetectable viral load. Regardless of the standard care quality, dominantly African-American or Hispanic samples had on average a $23 \%(95 \% \mathrm{Cl}, 7 \%$ to $40 \%)$ lower chance of achieving virologic success than dominantly Caucasian samples. Figure 2 shows a scatterplot of standard care quality against the observed proportions corrected for the 
influence of the other four moderators (the size of the points is proportional to their weight in the analysis). The solid line indicates the predicted average proportion as a function of standard care quality and the dotted lines indicate the corresponding $95 \% \mathrm{Cl}$ bounds.

Table 2. Study details for the meta-analysis

\begin{tabular}{|c|c|c|c|c|c|c|}
\hline $\begin{array}{l}\text { Author } \\
\text { (Year) }\end{array}$ & $\begin{array}{l}\text { Standard } \\
\text { care } \\
\text { quality1 }\end{array}$ & $\begin{array}{l}\mathrm{N} \\
\text { (undetectable/ } \\
\text { detectable) }\end{array}$ & $\begin{array}{l}\text { Dominant } \\
\text { ethnicity } 2\end{array}$ & $\begin{array}{l}\text { Patients' } \\
\text { treatment }\end{array}$ & $\begin{array}{l}\text { Selection on } \\
\text { non- } \\
\text { adherence }\end{array}$ & $\begin{array}{l}\text { Viral load } \\
\text { sensitivity }\end{array}$ \\
\hline $\begin{array}{l}\text { Andrade } \\
\text { (2005) }\end{array}$ & 21,00 & $29(11 / 18)$ & Other & Starting & No & $<400$ \\
\hline $\begin{array}{l}\text { Fairley } \\
\text { (2003) }\end{array}$ & 22,673 & $33(24 / 9)$ & Caucasian & Continuing & Yes & $<400$ \\
\hline $\begin{array}{l}\text { Goujard } \\
\text { (2003) }\end{array}$ & 15,07 & $326(179 / 147)$ & Caucasian & Continuing & No & $<400$ \\
\hline $\begin{array}{l}\text { Holzemer } \\
(2006)\end{array}$ & 3,67 & $240(82 / 158)$ & Other & Continuing & No & 400 \\
\hline $\begin{array}{l}\text { Knobel } \\
\text { (1999) }\end{array}$ & 8,60 & $110(60 / 50)$ & Caucasian & Starting & No & $<400$ \\
\hline $\begin{array}{l}\text { Murphy } \\
(2002)\end{array}$ & 27,80 & $29(11 / 18)$ & Other & Continuing & Yes & 400 \\
\hline $\begin{array}{l}\text { Pradier } \\
(2003)\end{array}$ & 4,80 & $244(99 / 145)$ & Caucasian & Continuing & No & $<400$ \\
\hline $\begin{array}{l}\text { Rathbun } \\
\text { (2005) }\end{array}$ & 12,07 & $17(9 / 8)$ & Caucasian & Starting & No & $<400$ \\
\hline $\begin{array}{l}\text { Remien } \\
(2005)\end{array}$ & 20,00 & $215(88 / 127)$ & Other & Continuing & Yes & $<400$ \\
\hline $\begin{array}{l}\text { Servellen } \\
(2005)\end{array}$ & 7,80 & $138(26 / 112)$ & Other & Continuing & Yes & 400 \\
\hline $\begin{array}{l}\text { Tuldra } \\
(2000)\end{array}$ & 7,47 & $26(17 / 9)$ & Caucasian & Starting & No & 400 \\
\hline $\begin{array}{l}\text { Wagner } \\
(2006)\end{array}$ & 10,47 & $55(23 / 32)$ & Other & Starting & Yes & 400 \\
\hline $\begin{array}{l}\text { Wohl } \\
(2006)\end{array}$ & 22,80 & $54(40 / 14)$ & Other & Starting & No & 400 \\
\hline
\end{tabular}




\section{DISCUSSION}

The purpose of this study was to investigate whether standard care quality could be assessed in a reliable manner, whether it varied between studies, and whether these variations were related to clinically relevant differences in success rates among HIV-infected patients receiving HAART and standard care. The results indicate that the standard care quality instrument is a reliable tool and that standard care quality varied considerably between reviewed studies. In addition, standard care quality was significantly related to clinically relevant variations in treatment success rates: Patients' chances on an undetectable viral load depend to a large extent on the quality of standard care provided to them. These results suggest that not all patients in the Western world benefit fully from the available antiretroviral therapies, and that considerable increases in HAART (cost)effectiveness may be expected if standard care is optimized (Goldie et al., 2003). Moreover, these data indicate that variations in effect sizes (comparing intervention and standard care groups) between clinical trials may be substantially influenced by variations in the quality of standard care provided to control groups in these trials.

The findings suggest that future meta-analyses of HAART-adherence interventions need to assess and control for variability in standard care quality so that more accurate estimates of intervention effectiveness can be produced. Furthermore, as Wagner and Kanouse (2003) have also argued, the substantial variation in standard care quality may well apply to behavior change interventions in other fields than adherence to HAART. For example, in a meta-analysis of randomized controlled trials of psychological interventions to improve glycaemic control in patients with type 2 diabetes, the authors report sizeable variations in standard care delivered to control patients: It ranged from "one minimal education session by a nurse" to " 16 behavioral modification sessions by a multi-disciplinary team" (Ismail et al., 2004). This warrants meta-analyses of behavior change interventions in other fields to also assess and control for variability in standard care quality delivered to their control groups so that more accurate estimates of intervention effectiveness can be achieved. In addition, systematic analyses of standard care in these fields can be useful to determine the effects and content of optimal standard care for other treatments than HAART. The instruments developed in this study may aid scholars to assess standard care quality in their domains. 


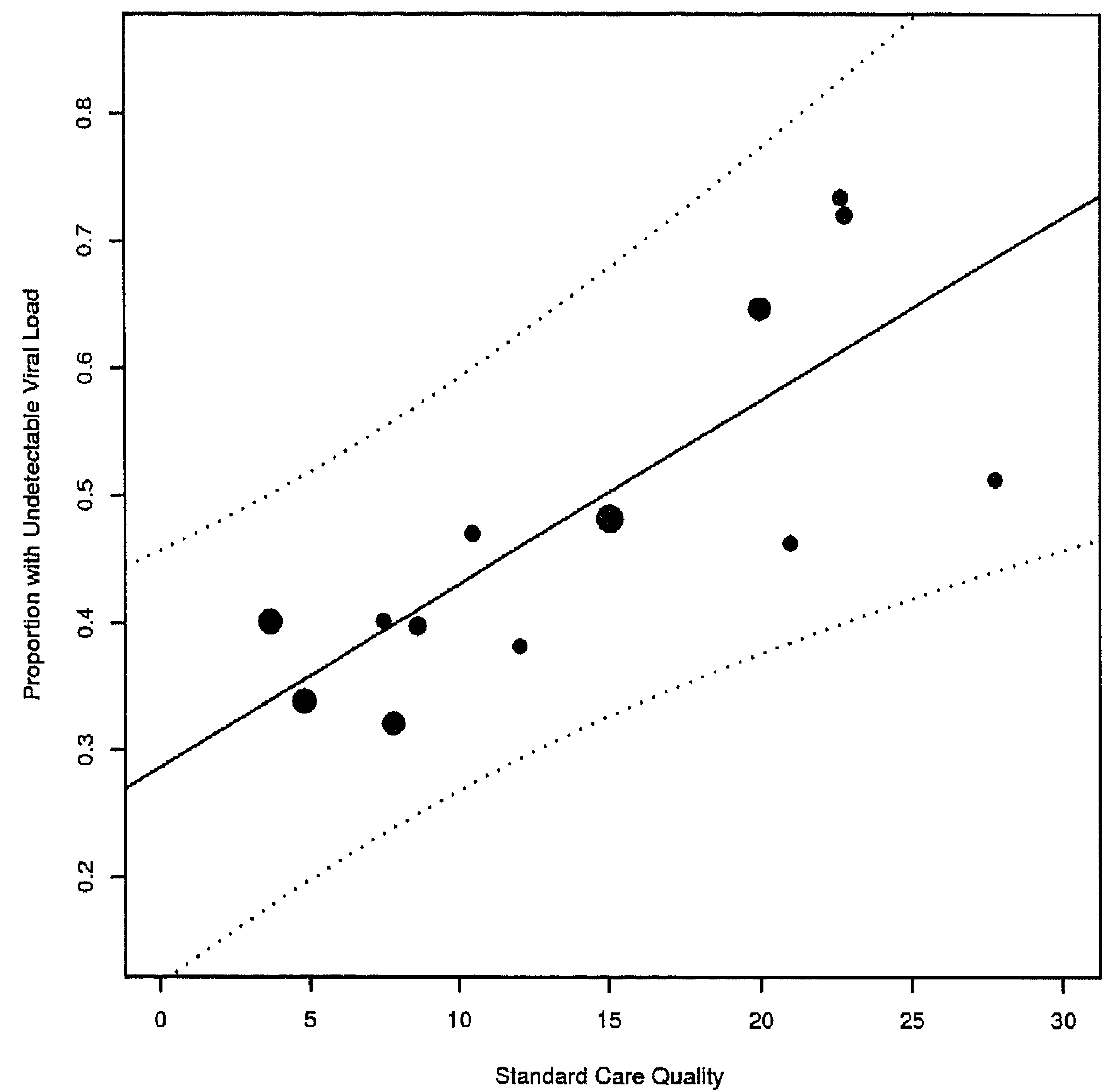

Figure 2. The proportion of participants with undetectable viral loads as a function of standard care quality. The Figure displays the proportions before (grey dots) and after correction (black dots) for the influence of the other four moderators.

The results of the present study also highlight the usefulness of ample descriptions of standard care quality in future publications of research trials. In the first place, this would enable those conducting meta-analyses to assess standard care quality directly from published articles. In addition, health care professionals would be able to compare the quality of standard care in their clinic to that delivered to a control group, allowing well-informed judgments about the probable effectiveness of an intervention in their clinic (Wagner \& Kanouse, 2003).

The results from the meta-regression model indicated that ethnicity was also a significant predictor of clinical success. In particular, samples with non-Caucasians as the dominant ethnicity had, on average, a $23 \%$ lower chance of achieving full viral 
suppression than samples with Caucasians as the dominant ethnicity. Although the evidence regarding differences in clinical response between ethnicities is mixed, several authors have suggested that individual and environmental differences could lead to lower adherence rates among non-Caucasian patients and thus to a less successful treatment (Golin et al., 2002; Halkitis et al., 2008; Nellen et al., 2004). Regardless of the reasons for the present result, it is important to note that both standard care quality and ethnicity are significant moderators when included simultaneously in the model. This suggests that the influence of these moderators is genuine and not attributable to some form of confounding between them (standard care quality was on average actually slightly higher in studies with non-Caucasians as the dominant ethnicity).

There are several limitations to this study. In the first place, our assessment was limited to a sample of HAART-adherence intervention studies. Therefore, our findings cannot be generalized to other fields. Furthermore, although 27 of the 29 authors of the selected articles for this study responded to our request for information, 9 were not able to provide sufficient information on the standard care delivered in their study, and 5 other studies could not be included in the meta-analysis. In addition, the standard care quality reports by the corresponding authors were retrospective which could have resulted in less accurate responses. Finally, other factors than effective techniques to influence behavioral determinants may contribute to standard care quality, such as the frequency and duration of contacts with health care professionals (Davidson et al., 2003).

The present research sought to develop useful instruments to assess the quality of standard care, to demonstrate that these instruments are sensitive to show variability in standard care quality, and that these variations explain clinically relevant differences in success rates in this domain. Future reviews and meta-analyses could investigate whether such variability is present in other domains, and - if so - include standard care quality as a moderator in the analysis, thereby providing a more accurate understanding of the effectiveness of behavior change interventions and techniques to improve patients' health and well-being. 


\section{CHAPTER 3}

\section{Appendix- Checklist of standard care activities}

This checklist was sent to all authors of selected studies to assess the content of standard adherence care. Authors completed the checklist and added any additional adherence activities that were not included in the checklist (see "Other [...], namely" at the end of each section). Two coders independently determined which of these activities were considered relevant, i.e. by coding which of the activities were adequate applications of effective behavior change techniques targeting important determinants of adherence. Hence, some activities were considered irrelevant for adherence, others were considered relevant and were therefore included in the standard care quality scores. Only the relevant activities are included in the checklist below.

Most of the content of this checklist has been included in Table 1 of the manuscript. However, the activity descriptions in the checklist are more extensive. Moreover, the coders merged some items in this checklist in 1 behavior change technique and 1 general activity. Table 1 contains the merged activities while the checklist contains all the separate practical activities (e.g. Information on HIV, medication and adherence is 1 activity in Table 1 but consists of 5 items in the checklist).

Each activity in the checklist below is followed by a number (e.g. 1) which refers to the coding of the activity described in the Table at the end of the document (i.e. similar to Table 1 in the manuscript).

Uses of the checklist

Health care professionals can examine the content and quality of their own standard adherence care, and determine whether there are additional activities they can adopt to increase the quality of their adherence care.

Researchers can use the checklist to assess standard adherence care quality in their study setting, and include the completed checklist in their publications. In case the researcher identifies additional standard adherence care activities in the study setting, please determine whether these are relevant before adding them to the checklist (coding manual available from the first author).

Researchers conducting reviews in other behavioral domains than adherence (to HAART) can use this checklist, and follow the procedure described in the manuscript, to develop domain-specific standard care checklists. These checklists can then be used to assess standard care activities, and code these activities to determine the quality and effectiveness of standard care in other domains. 
Below we present a summary of main characteristics of standard care procedures reported by authors of HAART adherence intervention articles. Some of these characteristics are mentioned often, others rarely. We would like to know which of these characteristics were part of the standard care procedures during your study [OR: that the participants in the control condition of your study received]. With standard care procedures we mean those characteristics of standard care that were provided systematically to all patients.

In Questions 3 thru 7 the answer options are 1. Yes, 2. No, and 3. ? (=don't know). Please check only one of these options.

The person(s) who educated the patient and discussed adherence during standard care was a:

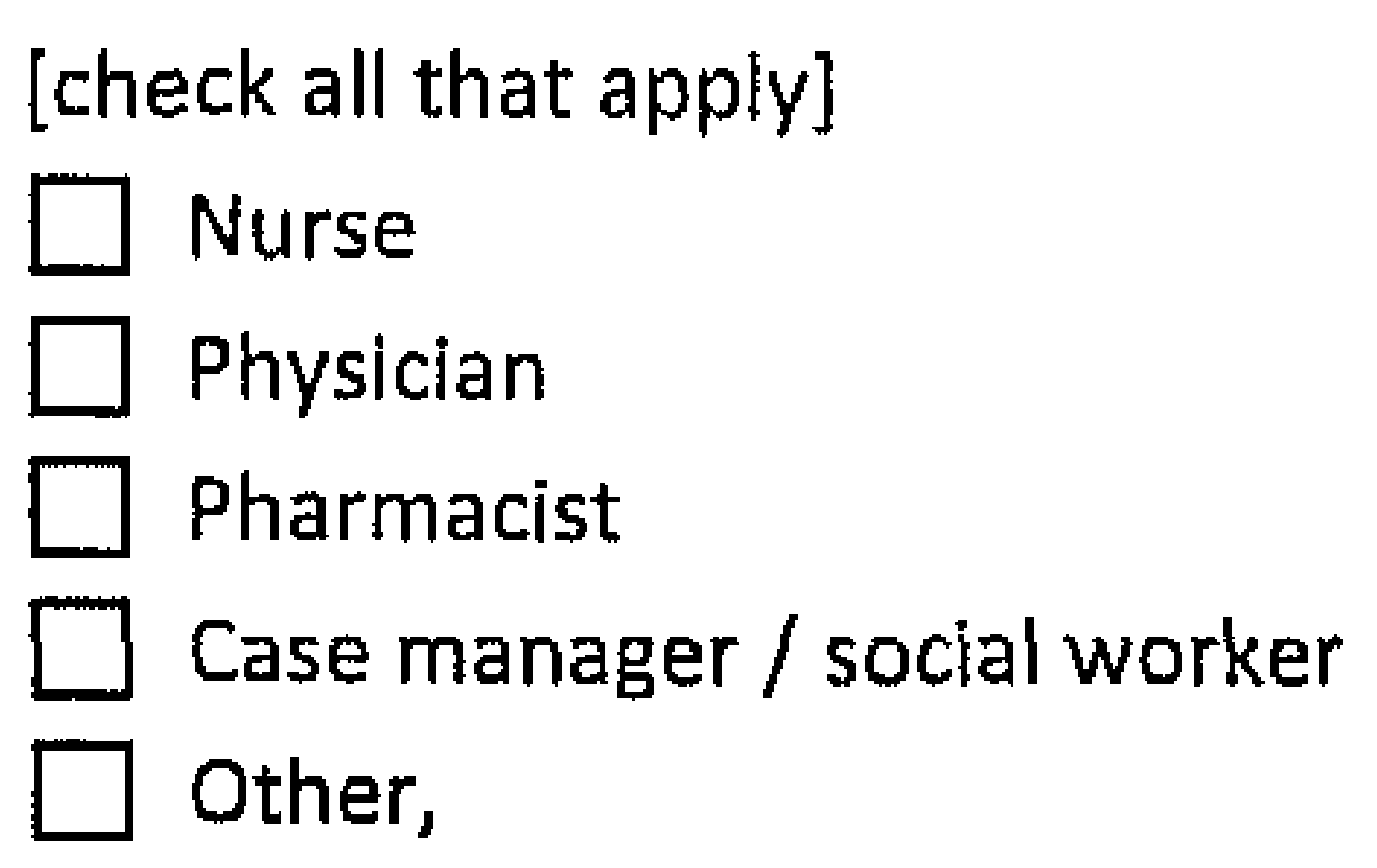

This person (these persons) are from now referred to as the healthcare provider(s) in the remainder of this questionnaire

Is the healthcare provider(s) trained (on-site by competent peers or through formal training) in patient education and counselling strategies?
$\square$ Yes, well-trained
$\square$ Yes, received some training
$\square$ No, received (almost) no training
$\square$ I do not know

The healthcare provider(s) delivered the following education about HIV and the medication systematically to all patients:

Yes No ?

What HIV is and what it does in the body (1)

The meaning of viral load and $\mathrm{CD} 4$ (1)

What the medicines do to fight HIV (1)

How the medication should be taken (1)

The importance of adherence and the consequences of non-adherence $(4,8)$

What to do if a dose is missed (1)

Common side-effects and how to deal with them (16)

Other education, namely 


\section{CHAPTER 3}

How was this education provided?

$\begin{array}{lll} & \text { Yes } & \text { No } \quad \text { ? } \\ \text { Verbal explanation to the patients* } & \square \quad \square \quad \square \\ \text { Use of illustrative materials (e.g., pictures, videos) (2) } & \square \quad \square \quad \square \\ \text { Handing out brochures or written information (3) } & \square \quad \square \quad \square \\ \text { Other media, namely } & \end{array}$

*Since counseling in all studies was done verbally, this was considered baseline and not given a quality score.

The healthcare provider assisted patients systematically and consequently in planning their medication intake by:

Encouraging patients to adhere to the treatment (8)

Developing a medication intake schedule for patients (11)

Tailoring the medication intake schedule to the patients' lifestyle (12)

Encouraging patients to identify daily cues (brushing teeth) and plan medication intake at these times (15)

Encouraging patients to use alarm devices to remember to take the medication (15)

Encouraging patients to use a 7-day pill box (5)

Encouraging patients to store spare doses of medication in different places (e.g. car, work, purse) (9)

Encouraging patients to plan ahead for holidays and weekends (10)

Encouraging patients to organize social support (14)

Discussing general barriers to adherence and ways to overcome them (10)

Writing down patients' dosing schedule (time, name of meds, number of pills) (13)

Using other approaches, namely 
During consecutive visits, it was standard care that:

The healthcare provider inquired about (possible difficulties with) adherence(10)

The healthcare provider and patient determined underlying causes of nonadherence (10)

The healthcare provider and the patient generated solutions for these probiems (10)

The healthcare provider inquired about side-effects. If necessary, appropriate steps were taken (16)

The healthcare provider gave feedback about the effectiveness of the treatment using viral load and CD4 results (6)

Feedback of MEMS-reports after a period of electronic monitoring of medication use (7)

The healthcare provider performed other actions, namely

General

Patients were always given a telephone number or beeper number and were told to contact the healthcare provider when they experienced problems with sideeffects (19)

Patients were always given a telephone number or beeper number and were told to contact the healthcare provider when they experienced problems with adherence (19)

Patients were always given a telephone number or beeper number and were told to contact the healthcare provider if they run of out medicines (19)

Follow-up telephone calls to inquire about difficulties with the treatment (19)

Patients with adherence problems were asked to return more frequently to their healthcare provider than patients who did not have adherence problems (21)

Social worker supports with housing, unemployment and legal issues (17)

Design a new regimen when the present regimen is too complex for the patient (18)

Delivery of medication at home (20)

Other aspects of standard care that were provided to your participants (e.g. adherence monitoring and feedback, self-monitoring):

Thank you very much for completing this checklist. 


\section{CHAPTER 3}

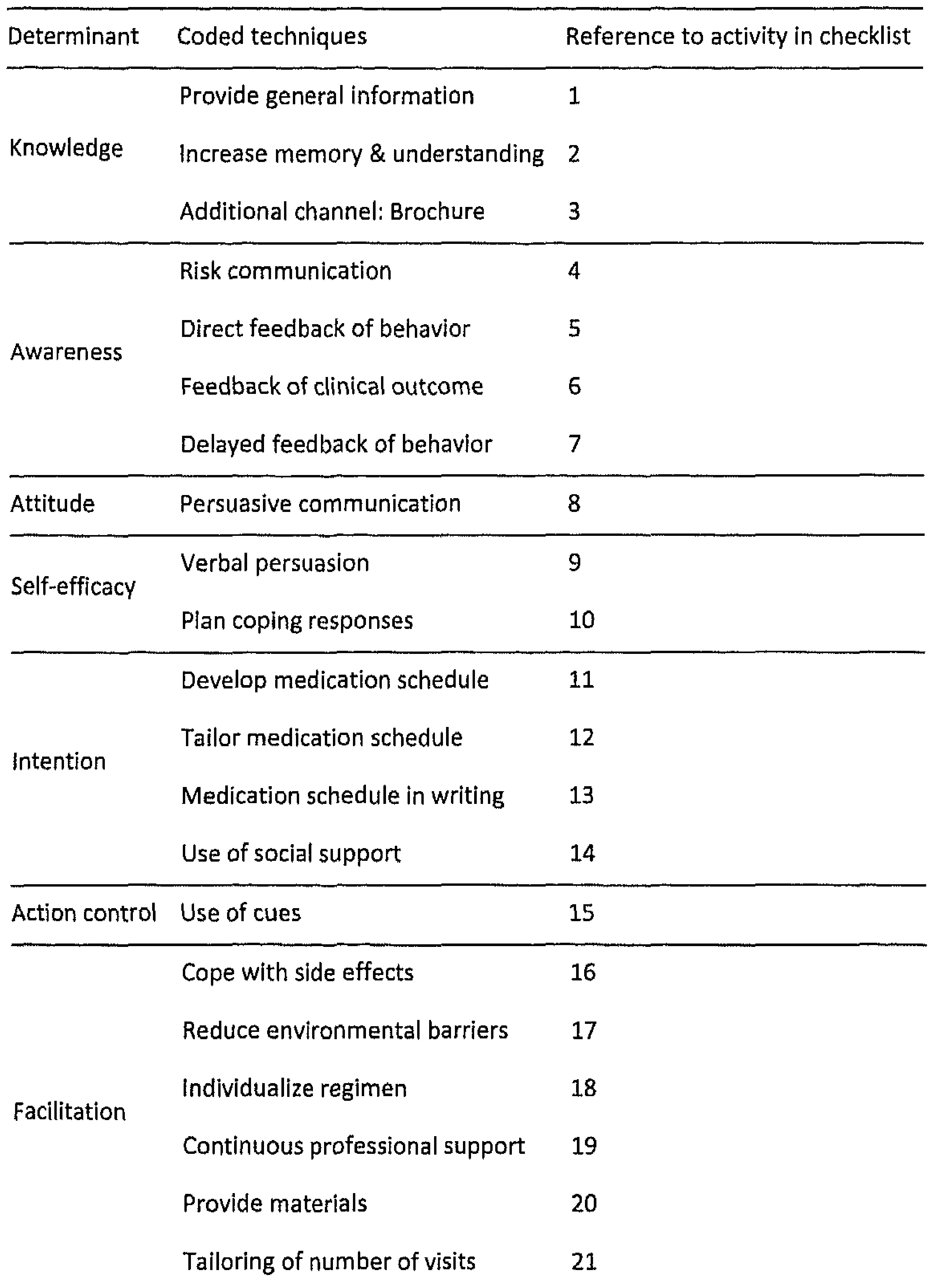

Note: Activities coded under 7, 21, 22, 23 and 24 have been reported by individual authors, were coded as applications of relevant behavior change techniques and therefore included in the checklist. 


\section{CHAPTER 4}

Electronic Monitoring-based Counseling to Enhance Adherence among HIV-infected Patients using HAART: A Randomized Controlled Trial

Marijn de Bruin, Harm J Hospers, Gerard JP van Breukelen, Gerjo Kok, William M Koevoets and Jan M Prins

Submitted 


\section{CHAPTER 4}

\section{ABSTRACT}

Background: Suboptimal adherence to HAART is an important cause of treatment failure and disease progression among HIV-infected patients. Evidence-based interventions that can be easily implemented in HIV-clinics are needed. This study investigated the effectiveness of an adherence intervention (AIMS) designed to fit HIV-clinics' routine care procedures.

Methods: Through block randomization, consenting patients were allocated to the intervention or the control group. The study included 2 months baseline measurement, 3 months intervention, and 4 months follow-up. Adherence was measured objectively with electronic pill-bottle caps (MEMS-caps) and viral load data were collected. HIV-nurses delivered a minimal intervention to patients scoring $>95 \%$ adherence at baseline, and an intensive intervention to patients with $<95 \%$ adherence. The control group received care as usual.

Results: 133 patients were included (67 control, 66 intervention), $60 \%$ had $<95 \%$ adherence at baseline, and $87 \%(116 / 133)$ completed the trial. Intent-to-treat analyses showed that adherence improved significantly in the complete intervention sample. Subgroup analyses showed that this effect was caused by the participants scoring $<95 \%$ at baseline (mean difference $=15.20 \% ; p<.001$ ). These effects remained stable during follow-up. The number of patients with an undetectable viral load increased in the intervention group compared to the control group (risk difference $[95 \% \mathrm{Cl}]=-0.11[-0.24,0.01]$; controlling for baseline viral load: $O R=$ $2.96, p<.05)$. The treatments effects on viral load were mediated by the improvements in adherence.

Conclusions: The AIMS-intervention was effective and has the potential to become an integral part of routine clinical care for HIV-infected patients. 


\section{INTRODUCTION}

Since the introduction of Highly Active Antiretroviral Therapy (HAART) in 1996, HIVinfection transformed from a life-threatening into a chronic disease. However, it was soon discovered that for HAART to suppress viral replication and remain effecttive over the years, high levels of patient adherence were required (Howard et al., 2002; Knobel et al., 2002; Knobel et al., 2001; Paterson et al., 2000). Given that a considerable proportion of the patients showed suboptimal adherence, leading to disease progression and treatment escalation (Bangsberg et al., 2003; Gross et al., 2006; Howard et al., 2002; Knobel et al., 2002; Knobel et al., 2001; Liu et al., 2006; Paterson et al., 2000; Perno et al., 2002), optimizing patient adherence to HAART became an important health care objective in the treatment of HIV.

In order to provide health care professionals with effective tools for supporting adherence, researchers developed and investigated the effectiveness of behavioral interventions to enhance adherence to HAART. Some studies have shown promising effects on adherence and clinical outcomes (Amico et al., 2006; Haynes et al., 2008; McDonald et al., 2002; Rueda et al., 2006). However, many were non-randomized, small-scale and short-term pilot-studies, measured adherence subjectively over brief time periods (e.g. 3-day self-reports), and/or experienced considerable dropout (>20\%; Haynes et al., 2008; McDonald et al., 2002; Rueda et al., 2006). Of the intervention studies without these methodological limitations, intervention programs were often delivered by specially trained personnel who complemented patients' usual care for the duration of the study. It is therefore unlikely that the resources required for these interventions (e.g. facilities, time, and competences) are readily accessible in routine clinical care.

In the present study we investigated the effectiveness of a theory- and evidence based behavior change intervention designed to fit in routine care procedures of HIV-clinics. This so-called Adherence Improving self-Management Strategy (AIM-Strategy) was developed in collaboration with health care professionals and pre-tested (de Bruin et al., 2005). The pilot-study showed promising effects on adherence and the program was positively evaluated by both patients and health care professionals. We therefore decided to study the effects of AIMS in a 9-month randomized controlled trial in which patients were assigned to receive either routine care or the AIMS-intervention, both during standard clinic visits. 


\section{CHAPTER 4}

\section{METHODS}

\section{Study setting and eligibility criteria}

Patients visiting the HIV outpatient clinic of the Academic Medical Center in Amsterdam, the Netherlands, between September 2005 and January 2007 were asked to participate. Patients were eligible if they were older than 18 years, treatment-experienced ( $>6$ months on HAART), started antiretroviral treatment not earlier than 1996, had no known psychiatric disorders, were not addicted to hard drugs or alcohol (stable methadone treatment was no exclusion criterion), and willing to follow the study visiting schedule. Considering the novelty of AIMS for the HIV-nurses delivering the intervention, we decided to avoid additional language and cultural barriers and focus this first large trial on Caucasian, Dutch-speaking patients (main patient population in the Netherlands). HIV-nurses and physicians approached all eligible patients during routine visits and provided verbal and written information about the study. The study was approved by the hospital ethics committee and all patients provided written informed consent. This trial is registered at www.controlled-trials.com (ISRCTN97730834).

\section{Study design and measurements}

Patients were explained that the study concerned a new form of adherence counseling and that they were required to use a MEMS-cap (AARDEX, Itd.) to monitor their medication intake. A MEMS-cap is a pill-bottle cap that fits on standard medication bottles and electronically registers bottle openings. These data can be downloaded and printed in reports that provide a detailed but comprehensible overview of medication intake behavior (see Figure 1). MEMS-data have been found to consistently predict clinical outcomes in the treatment of HIV (Farmer, 1999; Liu et al., 2001; Paterson et al., 2002). Moreover, MEMS-data can be collected continuously and over long time periods, which increases reliability of the assessments.

Patients who decided to participate commenced a two-month baseline adherence assessment period using the MEMS-cap. At the end of this period, patients completed a questionnaire to assess demographic variables and depression scores (Beck Depression Inventory-II). Participants then returned to the hospital and got randomized to either the intervention or the control condition. We used block randomization (block size: 6) with a 1:1 ratio to ensure an equal distribution of intervention and control participants over the seven HIV-nurses. The two-month 
delay between inclusion and randomization made it essentially impossible to predict a participant's treatment allocation until interventions were assigned.

With the usual interval between visits ranging from 3-4 months in this clinic, we chose for a 3-month intervention, and a 4-month follow-up period. During the intervention period, patients received standard care or the AIMS intervention, and continued using the MEMS-cap. The HIV-nurses recorded the duration and content of each intervention contact. During follow-up, patients were allowed to interrupt MEMS-use during the first two months, but were required to recommence MEMSuse during the last two months. The effects of the intervention were evaluated using three 2-month periods of adherence measurement: baseline (month 1-2), intervention (month 4-5), and follow-up (month 8-9).

After month nine, patients in the intervention group completed an evaluation questionnaire, and HIV-nurses were interviewed to assess their experiences with the intervention.

\section{Procedure for optimal MEMS-use}

Although the MEMS-cap is a reliable measurement instrument, it may underestimate adherence in case of suboptimal use (e.g., pocket dosing; Fennie et al., 2006; Liu et al., 2001; Miller \& Hays, 2000; Paterson et al., 2002; Wendel et al., 2001). During the pilot-study we learned this occurred especially among patients who preferred to carry a small, pocket-size bottle in social situations and among those using week boxes. We therefore incorporated additional control procedures to optimize the quality of the MEMS data and representativeness of the study sample (for details see Appendix I).

\section{Standard care provided to the control group}

We used the instrument developed by de Bruin and colleagues to assess the quality of standard adherence care provided to the control group, which showed that the quality in the study clinic was high (see Appendix II; de Bruin et al., in press). In brief, nurses provided verbal and written information about the treatment and about the consequences of (non)adherence, the medication regime and intake schedule were tailored to patients' daily life, side-effects were closely monitored and dealt with if possible, the use of helpful devices (week organizer, alarm devices) was promoted, adherence problems and solutions were discussed, and these techniques were repeatedly applied during follow-up consultations (i.e., usually every 3-4 months). In 


\section{CHAPTER 4}

addition, patients received feedback about their viral load and CD4 count, and those with complex problems were asked to return more frequently.

\section{The AIMS Intervention}

The AIMS intervention has been described in detail previously, including the theoryand evidence-based rationale (de Bruin et al., 2005). We will only briefly discuss its content here.

After the two month baseline measurement, the HIV-nurse downloaded the data from the MEMS-cap and judged patients' adherence. If adherence was $\geq 95 \%$, patients received a "minimal" intervention (i.e., adherence sustaining), comprising feedback of MEMS-reports, reinforcement, and a brief discussion to see whether patients experienced any difficulties that could be solved. The full intervention was provided to patients with $<95 \%$ adherence at baseline (i.e., adherence improving). This started with HIV-nurse providing tailored information about adherence (verbally and using graphs), and asking patients to select a desired adherence level using exemplar MEMS-reports. These varied from perfect to low adherence, and patients were encouraged to choose a report with sufficient yet feasible levels of adherence, and to motivate their choice. The HIV-nurse and patients then examined patients' own MEMS-reports. If adherence was suboptimal compared to patients' desired level of adherence, the HIV-nurse and patients identified the causes of the non-adherent events visible in the MEMS-reports, and identified tailored solutions for these problems. Finally, patients were asked to actively self-monitor their medication intake during the upcoming period in order to identify challenging situations, and to develop solutions to cope with the challenges. During the next intervention visit (usually 3 months later, but patients with extensive adherence problems could have an additional visit halfway) patients' difficulties and efforts were discussed, new MEMS-reports were examined, and if necessary action plans adapted. If sufficient change had occurred, goals for maintenance of behavior were formulated. The target of the intervention was to aim for optimal but sustainable adherence levels for each individual patient (e.g., $>90 \%$ for the one, $>95 \%$ for the other).

The HIV-nurses also discussed the MEMS-reports with the control participants at the end of the study and delivered the intervention when adherence was suboptimal. 
AIMS RCT

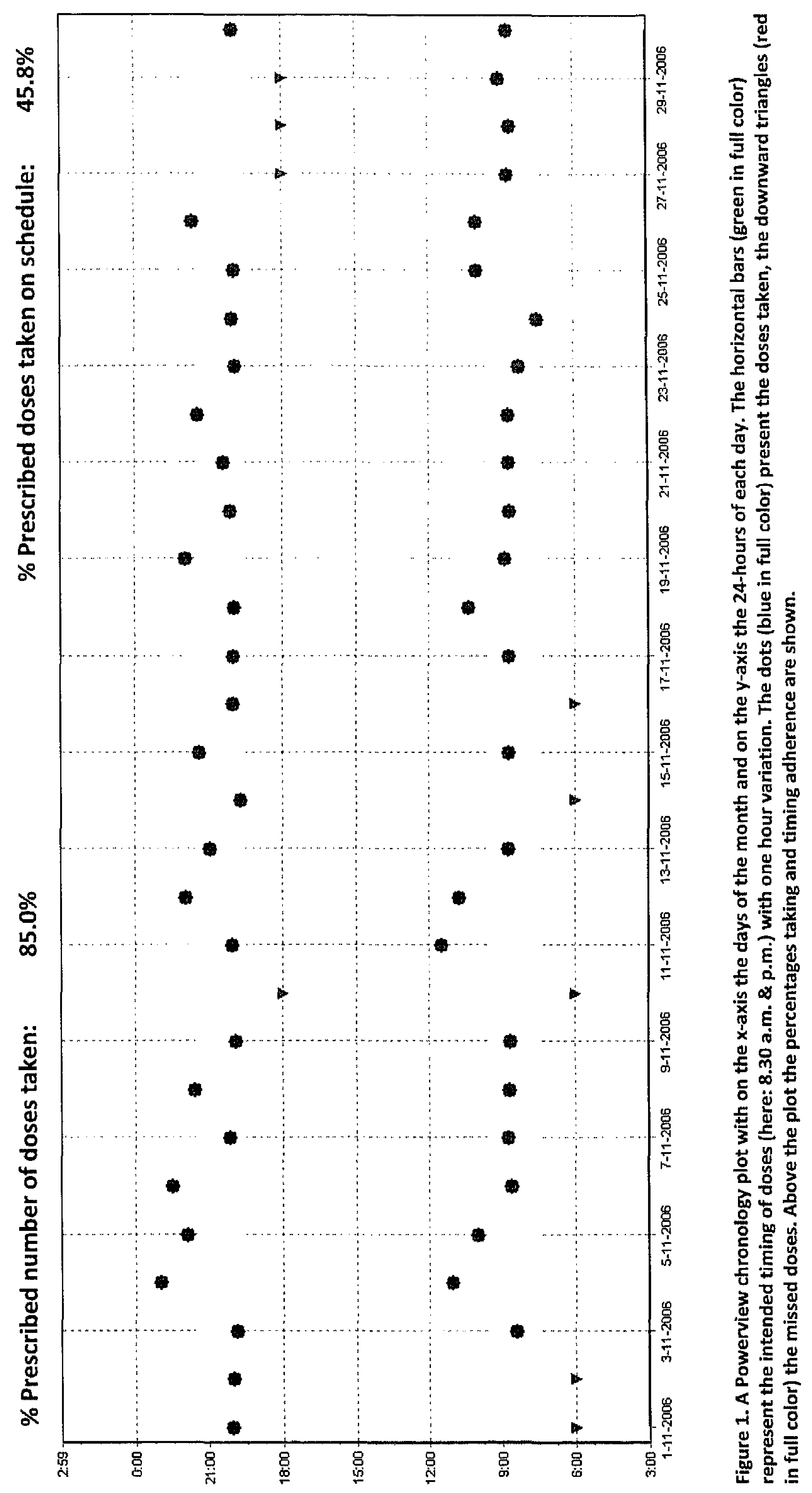




\section{Outcome measures, statistical analyses and sample size}

Adherence was primarily evaluated in terms of timing adherence, i.e. the percentage of doses taken within a specified time interval (11-13 hours for twicedaily and 22-26 hours for once-daily regimens). A secondary outcome measure was taking adherence (percentage of prescribed doses taken). Mean baseline (month 12), intervention (month 4-5) and follow-up (month 8-9) adherence scores were computed over 8-week periods. Treatment effects were analyzed using repeated measures ANOVA, with treatment as between-subject factor and time (intervention, follow-up) as within-subject factor, and baseline timing adherence as covariate to increase power (van Breukelen, 2006). If no Treatment by Time interaction was found (i.e. from intervention to follow-up), treatment effects were analyzed averaged across both periods. Adherence analyses were done for the total sample and, in case of a significant Treatment by Baseline adherence interaction, for the intensive and minimal intervention separately.

The adherence analyses were conducted on all complete cases and with an intention-to-treat procedure for patients dropping out (last observation carried forward). We also conducted a mixed linear regression that mimics the repeated measures ANCOVA (for details, see Van Breukelen, 2006), using only the available data (i.e., without imputing missing values).

Viral load was dichotomized (plasma HIV-RNA < or $\geq 50$ copies $/ \mathrm{ml}$ ) and changes in viral load were evaluated in a logistic regression with baseline viral load as covariate. Both post-intervention time points (i.e. immediately post-intervention at month 5 and follow-up at month 9) were combined using the OR rule to gain power for the treatment effect evaluation. Thus, patients were classified as having a detectable viral load if it was detectable on at least one of both time points. Since viral loads were collected during standard care, most outcomes were also available for patients who dropped out the study. In an additional intent-to-treat analysis missing values at post-intervention were replaced by patients' last available observation.

The required sample size was calculated for patients receiving the intensive intervention ( $<95 \%$ timing adherence), using data obtained during the pilot-study (de Bruin et al., 2005). Based on our primary outcome timing adherence, two-tailed testing with $\alpha=0.05$ and a power $1-\beta=0.90$, a standardized effect size (Cohen's $d=0.70$ ), and an anticipated dropout of $15 \%$, per group 50 participants with $<95 \%$ timing adherence at baseline were required. Since this power calculation does not account for the power increase resulting from including baseline adherence as covariate (van Breukelen, 2006), a sample size of 40 patients with $<95 \%$ adherence per condition was considered satisfactory. 


\section{RESULTS}

Of the 348 patients screened between September 2005 and January 2007, the time frame set for study inclusion, 133 patients were included and randomized. Hundred sixteen patients $(87 \%)$ completed the study (Figure 2). Patients who dropped out were more likely to be randomized to the control condition and had slightly lower adherence at baseline.

The participants were predominantly male with a mean age of 48 (range 21-74) years. Other baseline characteristics are given in Table 1 . Sixty percent (39/67 control patients and 41/66 intervention patients) scored below $95 \%$ timing adherence at baseline. Thus, 41 intervention patients received the intensive, and 25 the minimal intervention.

\section{The effects of the intervention on adherence}

In the intent-to-treat analysis of the complete sample $(n=133)$, the repeated measures ANOVA showed no Treatment by Time interaction (i.e. from intervention to follow-up). The following results are therefore based on ANCOVA with group and baseline as independents, and adherence averaged across both the intervention and follow-up periods as dependent. The effect of the intervention on timing adherence was significant $(p<.001)$. However, a significant Treatment by Baseline Adherence interaction indicated that depending on participants' baseline adherence, the effects of the intervention differed. When we therefore examined the two subgroups separately we found that the minimal intervention delivered to patients with $>95 \%$ adherence at baseline did not result in a better medication intake, since adherence remained stable in both conditions $(p=.62)$. In contrast, the participants who scored $<95 \%$ baseline adherence and received the intensive intervention showed superior levels of timing adherence after the intervention compared to the control participants with $<95 \%$ baseline adherence $(F(1,77)=$ $19.94, p<.001$, mean difference $=15.20$ [8.42-21.98]). These findings were similar in the analyses of taking adherence, with only the intensive intervention group displaying significant improvements in adherence $(F(1,77)=16.80, p<.001$, mean difference $=6.51$ [3.35-9.68]; see Figure $3 a-d)$. The complete case analyses and the mixed regression gave essentially similar effects as the ANCOVA described above. 


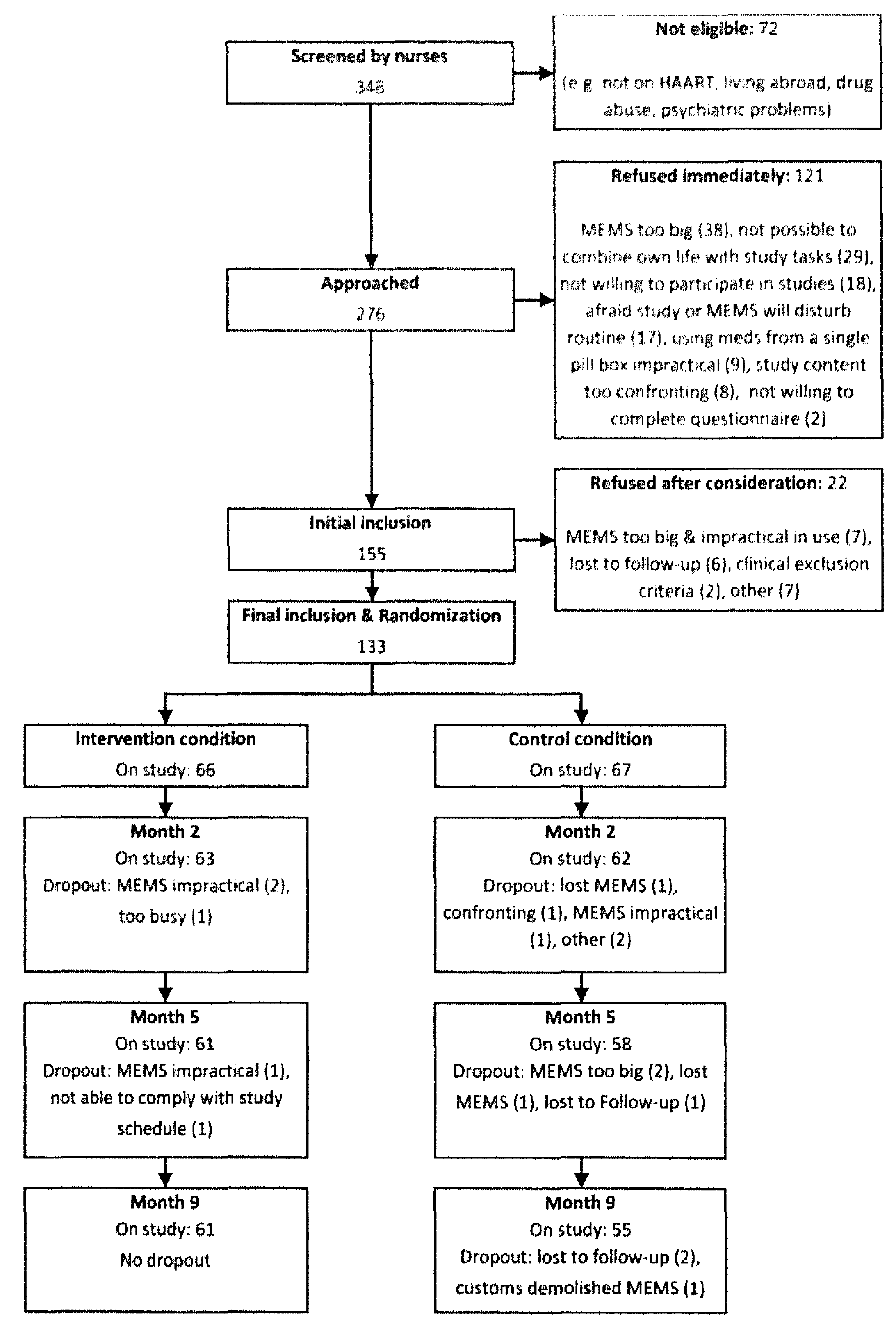

Figure 2. Flow of the participants through the study 
Table 1. Baseline characteristics of study participants

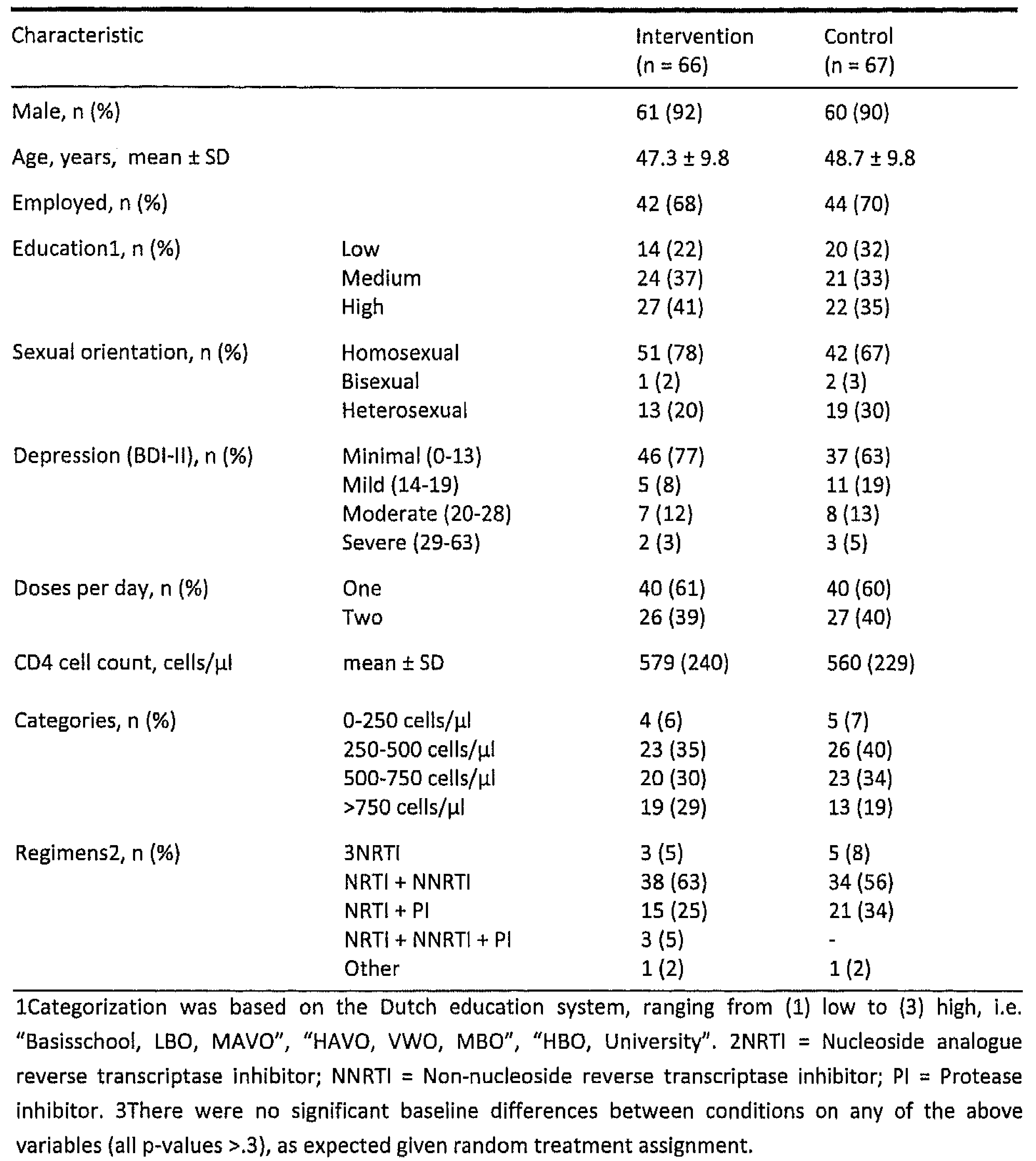

\section{Changes in viral load}

Due to the high level of standard care in this clinic, most patients had an undetectable plasma viral load at baseline: 53/66 (80\%) intervention and 59/67 (88\%) control participants. Baseline and post-intervention data were available for 120 patients. Controlling for baseline viral load, the logistic regression showed that the intervention group had a higher chance of being undetectable at post-intervention 


\section{CHAPTER 4}

than the control group $(p<.05, \mathrm{OR}[95 \% \mathrm{Cl}]=3.32[1.13-9.80])$. When missing values at post-intervention were replaced by the last available observation (i.e. all undetectable), similar effects were observed $(n=127, p<.05$, OR $[95 \% \mathrm{Cl}]=2.96$ [1.00-8.74]; see Table 2 for viral load data). Mediation analyses (for details on procedure, see Mackinnon et al., 2002), showed that the observed relation between treatment condition and post-intervention viral load was fully mediated by the change in adherence from baseline to post-intervention (both in terms of taking and timing adherence; details not reported). Hence, intervention effects on viral load appear to be due to the combined effects of the intervention on adherence and of adherence on viral load.

There was no significant Treatment by Baseline viral load interaction and subgroup analyses were therefore not conducted.

\section{Delivery of the intervention}

Both the interviews with the HIV-nurses and the study dossier data suggest that overall the main intervention components were adequately delivered. The duration of the intervention contacts took on average thirty minutes (mean $30 \pm 15$ minutes) at month 2, while the consultation at month 5 took considerably less time (mean 18 \pm 9 minutes). Six intervention participants returned for a brief additional visit between month 2 and month 5 (mean $18 \pm 12$ minutes). Control patients also followed the usual 3-month visit interval. These consultations usually last 15 minutes, which may increase up to 30 minutes or more when complex problems need to be discussed.

\section{Patients' evaluation of the intervention}

Fifty intervention participants completed an evaluation questionnaire about the user-friendliness of the MEMS-cap (scale 1-10, mean $6.7 \pm 2.8$; moderately positive), and the usefulness of the MEMS-reports and the intervention sessions with the HIVnurses (both mean $8.2 \pm 1.5$; very positive). Patients were also asked what MEMSdesign they would prefer. Most suggestions were to have a design with multiple compartments (similar to a week box) and a smaller, flat, pocket-size pill box for day doses. 

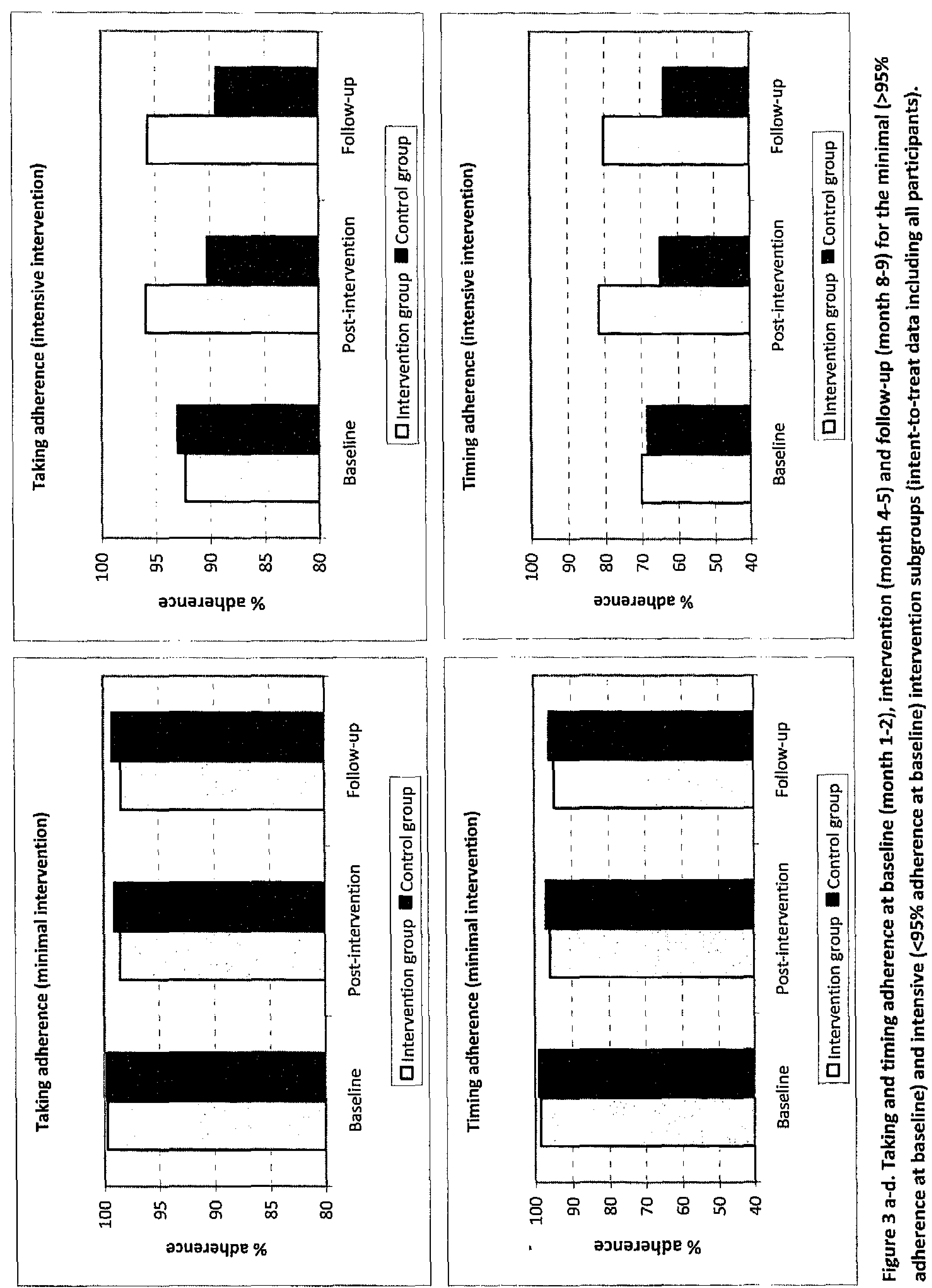
Table 2. Plasma viral load before and after the intervention, split by group and baseline viral load (intent-to-treat data)

Plasma viral load (copies/ml)
\begin{tabular}{|l|l|l|l|l|}
\hline \multicolumn{3}{|c|}{ Post-intervention $^{1}$} \\
\hline \multirow{3}{*}{ Intervention group at baseline } & $>50$ & $>50$ & $<50$ & Total \\
\cline { 2 - 6 } & $<50$ & 2 & 11 & 13 \\
\cline { 2 - 6 } & Total & 4 & 47 & 51 \\
\hline \multirow{3}{*}{ Control group at baseline } & $>50$ & 4 & 58 & 64 \\
\cline { 2 - 6 } & $<50$ & 9 & 4 & 8 \\
\cline { 2 - 6 } & Total & 13 & 50 & 63 \\
\hline
\end{tabular}

Detectable viral load ( $>50$ copies $/ \mathrm{ml}$ ) at post-intervention or at follow-up is coded as detectable. Undetectable viral load ( $<50$ copies $/ \mathrm{ml}$ ) at both time-points is coded as undetectable. Note: Missing from the Table are 2 intervention and 3 control group participants without baseline viral load data, and 1 control participant without baseline or post-intervention data.

\section{DISCUSSION}

The purpose of the present study was to evaluate the effectiveness of a behavior change intervention to enhance adherence among HIV-infected patients using HAART. The intervention was developed to fit standard care procedures and AIMS was tested against a control condition that received high quality standard care. Nevertheless, the treatment group showed a considerable improvement in adherence during the intervention, which remained stable during follow-up. The effectiveness of the intervention was also visible in a decrease of the percentage of patients with a detectable viral load in the intervention compared to the control group, an effect that was mediated by the changes in adherence. Hence, this randomized study of an adherence intervention tailored to HIV-clinic's usual care procedures is one of the few to show positive effects on both adherence and viral load.

The AIM-Strategy is founded on theory and evidence, has been developed together with those implementing the intervention, and has been pilot-tested. This approach is compliant with Intervention Mapping principles, a protocol for the systematic development of health promotion programs (Bartholomew et al., 2006). The effectiveness of AIMS has now been demonstrated in a randomized controlled trial. Since the intervention can be adequately delivered during routine clinical visits, the implementation of AIMS in standard clinical care should require only 
minimal investments lat least, in settings where HIV-nurses or pharmacists complement medical care): training of new personnel and the expenses of MEMScaps (i.e., 40 US\$ per cap per year). Finally, given that these effects were achieved against a control group that already received high-quality standard care, the effectiveness of AIMS should be similar or larger in most other HIV-clinics (de Bruin et al., in press; Wagner \& Kanouse, 2003).

The use of detailed MEMS-reports in the intervention was a core element of AIMS and was appreciated by both patients and health care providers. Unfortunately, especially for patients taking their medication in a public environment or those using a week box, the MEMS-design is neither discrete nor practical. Since this was anticipated, we devised additional strategies for MEMS-use (e.g. corrections for occasional pocket-dosing). What these strategies could not prevent is that $20 \%$ of the eligible patients reported to decline participation because of the MEMS-design. Similar problems with MEMS-acceptance among HIV-positive patients have been encountered by others (personal communication with D. Burger, PhD; Wendel et al., 2001). Although the percentage of patients declining participation was similar to other HAART-adherence intervention trials (Golin et al., 2006; Milam et al., 2005; Murphy et al., 2002; Wohl et al., 2006), the final study sample may not fully represent the population. This limitation extents to the Non-Caucasian patients that were not included in this first, large-scale study of AIMS. A final limitation was that the HIV-nurses delivering the intervention were also the ones providing the standard care. It is conceivable that the quality of standard care further improved through their experience with the AIMS-intervention.

In this setting with already high quality standard care, patients receiving the AIMSintervention successfully increased their adherence and viral suppression compared to the control participants. Both patients and health care professionals evaluated the intervention positively, and AIMS largely fits in routine clinical care. These encouraging findings warrant further study of this intervention in settings with different levels of standard care and among more heterogeneous samples (e.g., including ethnic minorities and drug addicted patients). More user-friendly MEMS designs, i.e. a week organizer and a pocket-size pill box, should facilitate the use of electronic monitoring of adherence in future studies, and smooth the progress of implementation of AIMS-like interventions in standard clinical care for HIV-infected patients. 
Amption:

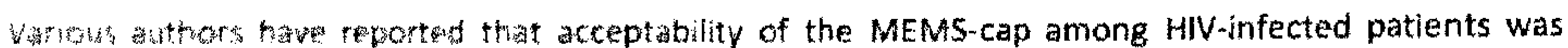

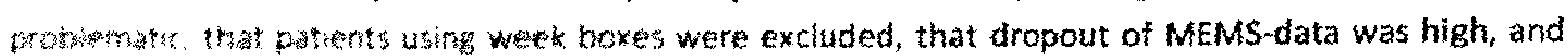

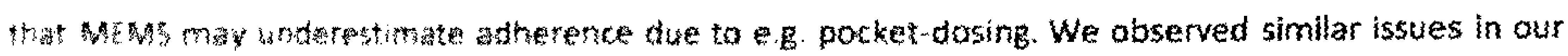
aritot stidy and made it a prionty to include a representative sample of patients in the trial, to closely

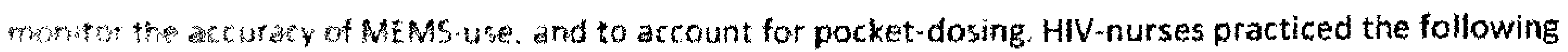
from

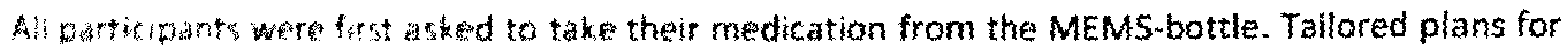

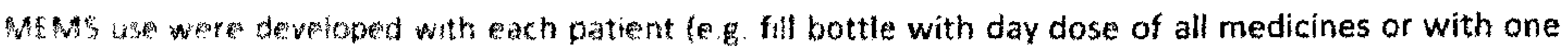

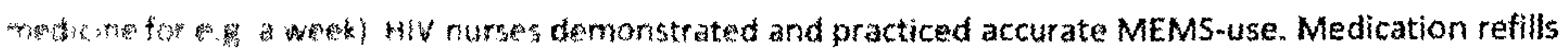

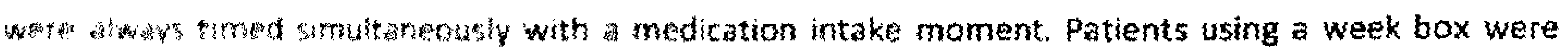

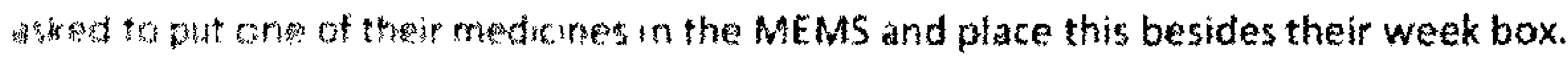

3. (A)

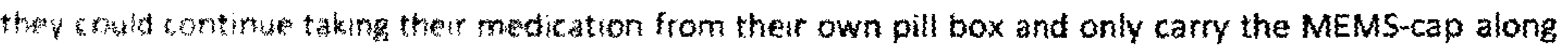

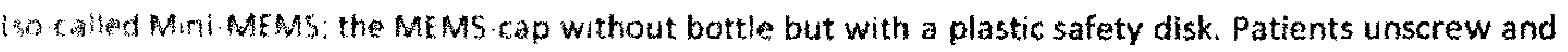

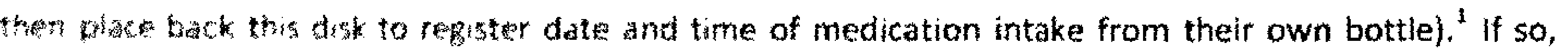

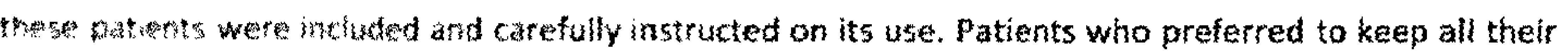

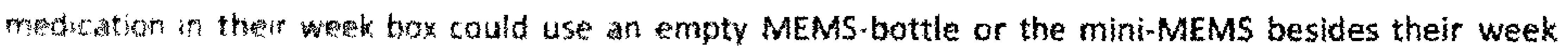
was

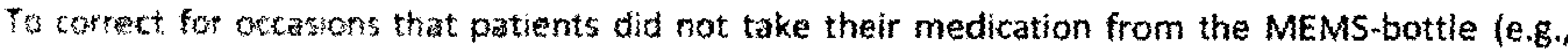

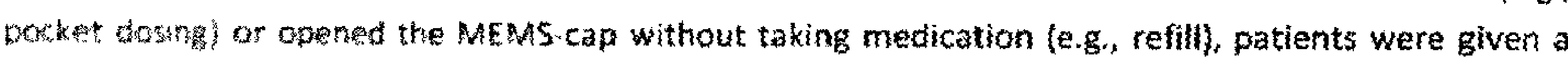

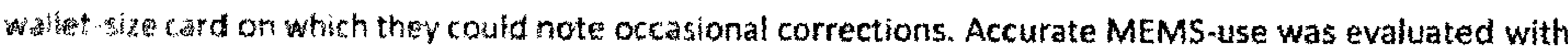
a

\section{Additionat analyses: MEMS-bottle versus mini-MEMS}

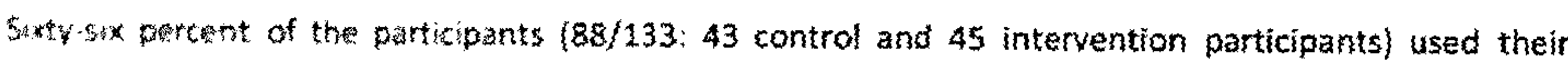
madcation ditecty from the MEMSbottle. In the repeated-measures ANOVA analyses, we included a

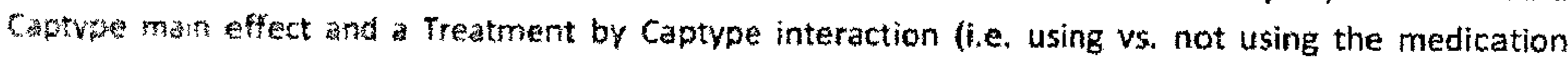

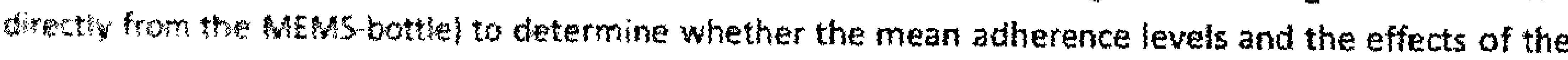
inturventyon depended on how patients used the MEMS. Neither the interaction term not the main sfoct of catyp apptoachad significance and were therefore removed from the analyses reported in

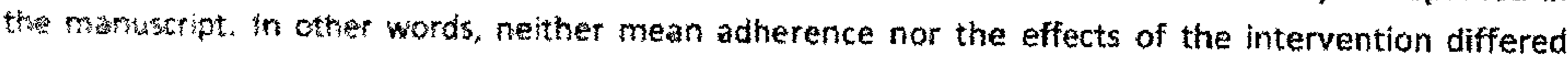

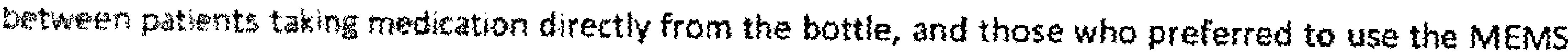
Tor regristration of intake only.

Peprentativenes of study samples in MEMS-studies among HIV-positive patients could be

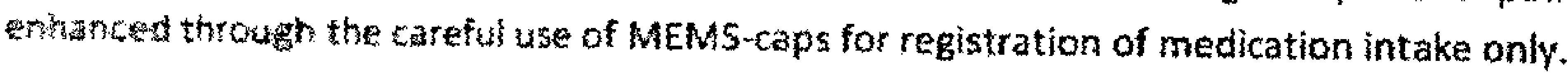




\section{Additional analyses: Accuracy MEMS-use}

As we also observed in our pilot-study, patients may temporarily use the MEMS-cap infrequently or inaccurately leading to underestimations of actual adherence (i.e., missing data). Despite the instructions described above, we expected this to occur in the present study as well. We therefore checked MEMSuse at each visit and discussed MEMS-reports with all patients (with control patients at the end of the trial). Approximately $10 \%$ of the patients (14/133) reported to have used the MEMS-cap infrequently or incorrectly for some time (mean duration $2.2 \pm 1.2$ months), which was also clearly visible in their MEMSdata but not in their viral loads (i.e., a sudden drop in adherence to levels essentially too low for full viral suppression: between $5-70 \%$ of the doses taken).Self-reported inaccurate MEMS-use occurred more frequently in the control than in the intervention group (10/67 control, 4/66 intervention patients). We therefore conducted additional analyses to examine the impact of these periods.

1. In the complete case analyses (repeated measures ANOVA), no corrections were made for periods of inaccurate MEMS-use.

2. In the intent-to-treat analysis using repeated measures ANOVA, the adherence recording of an inaccurate MEMS-period was replaced by 1 . the mean adherence during the month before and after the unreliable period, or 2. the last accurate observation (1-month mean) if no data was available after the inaccurate period, or 3 . the first accurate observation (1-month mean) if no data was available before the inaccurate period.

3. In the mixed-effects regression analyses, unreliable periods were treated as missing data.

Correcting for periods of inaccurate MEMS-use reduced the size of the intervention effect (e.g., from a mean difference of $20 \%$ to $15 \%$ ) but also the standard error, so that all analyses yielded approximately similar intervention effects. Only the ANOVA intent-to-treat analyses have therefore been reported in detail in the manuscript.

Future MEMS-studies among HIV-infected patients could benefit from systematically assessing the accuracy of MEMS-use, including discussions of the MEMS-results with each patient at the end of the study.

${ }^{1}$ Additional reference: Murphy, D. A., Marelich, W. D., Rappaport, N. B., Hoffman, D., \& Farthing, C. (2007) Results of an Antiretroviral Adherence Intervention: STAR. Journal of the International Association of Physicians in AlDS Care, 6(2), 113-124. 


\section{Appendix 销}

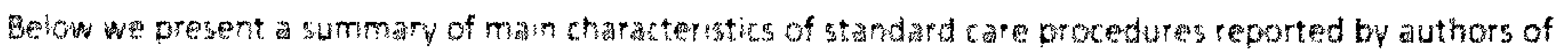

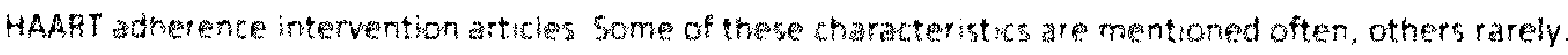

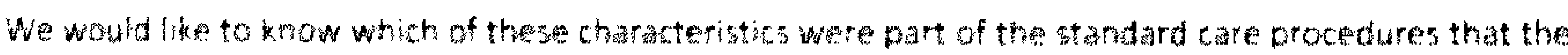

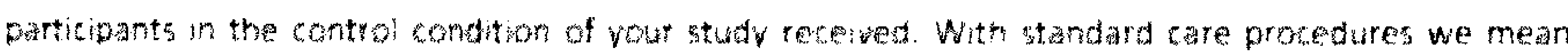

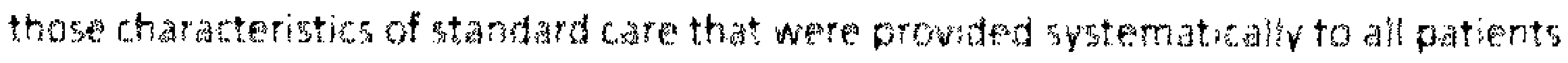

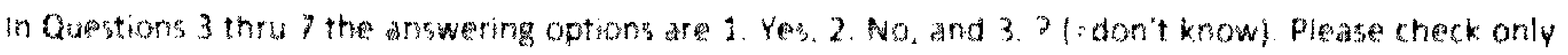
one of there optorsts

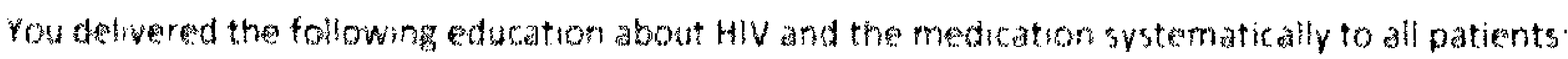

What Hiv is and what it doas in the body

The maning of vir alload and CD4

What the medicines do to fight HW

The importance of adherence and the consequences of non-adherence

$\otimes$

What to do if a dose is misned

田

Common side effects and how to deal with them

$\nabla$

Druginteractions

Drug storage recommendations

Other education, namely: How to handle the medications when travelling to another time zone

How was this education provided?

Other media, namely 
You assisted patients systematically and consequently in planning medication intake by:

Encouraging patients to adhere to the treatment
Developing a medication intake schedule for patients
Tailoring the medication intake schedule to the patients' lifestyle
Encouraging patients to identify daily cues (brushing teeth) and plan medication intake
at these times
Encouraging patients to use alarm devices to remember to take the medication
Encouraging patients to use a 7-day pill box
Encouraging patients to store spare doses of medication in different places (e.g. car,
work, purse)
Encouraging patients to plan ahead for holidays and weekends
Encouraging patients to organize social support
Discussing general barriers to adherence and ways to overcome them
Writing down patients' dosing schedule (time, name of meds, number of pills)
The physician designed a new medication regimen in case patients find their present
regimen too complex
Using other approaches, namely

During consecutive visits, it was standard care that:

The healthcare provider inquired about (possible difficulties with) adherence $\quad \square$

The healthcare provider and the patient determined underlying causes of nonadherence

The healthcare provider and the patient generated solutions for these problems

The healthcare provider inquired about side-effects. If necessary, appropriate steps were taken

The healthcare provider gave feedback about the effectiveness of the treatment using viral load and CD4 results

The health care provider makes follow-up telephone calls after the initiation of HAART to inform about difficulties with the treatment

The healthcare provider performed other actions, namely 


\section{CHAPTER 4}

General

Patients were always given a telephone number or beeper number and were told to contact the healthcare provider when they experienced problems with side-effects

Patients were always given a telephone number or beeper number and were told to contact the healthcare provider when they experienced problems with adherence

Patients were always given a telephone number or beeper number and were told to contact the healthcare provider if they run of out medicines

Patients with adherence problems had to return more frequently to their healthcare provider than patients who did not have adherence problems

Medication of patients was delivered at their homes

Social worker supports patients in dealing with unemployment, legal issues, housing, etc

Other aspects of standard care that were provided to your participants (e.g. adherence monitoring and feedback, self-monitoring): 


\section{CHAPTER 5}

How Intentions Translate into Behavior: Preparatory and Self-regulation Activities Mediate the Intention-Behavior Relation in a Longitudinal Study of Medication Adherence among HIV-infected Patients using HAART 


\section{CHAPTER 5}

\section{ABSTRACT}

People's intention to perform a behavior is generally considered as the most immediate predictor of that behavior in the future. However, longitudinal studies show that intentions explain on average "only" about 30\% of variance in behavior. A few studies have identified factors that may help explain the limited predictive value of people's present intentions for their future behavior. The objective of the present study was to contribute to this emerging field by examining whether socalled instrumental activities (i.e., preparatory and self-regulatory acts) mediate the relationship between HIV-infected patients' intention to adhere to their medical regimen, and their actual adherence. This was examined in a longitudinal study among 63 treatment-experienced patients attending a control group in a randomized controlled trial. Cognitive predictors of intention, the adherence intention itself, and the instrumental activities were assessed using a questionnaire at Time 1 , while adherence was measured objectively with so-called MEMS-caps (i.e., electronic pill-caps that fit on standard medication bottles) at Time 2 . The results showed that two of the three instrumental activities, namely 'self-monitoring of medication intake' and 'carrying medication along when outdoors', simultaneously mediated the relationship between people's adherence intention and actual adherence. Hence, it seems instrumental acts can be a valuable addition to determinant studies and possibly to behavioral theories that consider people's intentions as the most proximate predictor of their health behavior. 


\section{INTRODUCTION}

Although people's intention to perform a health behavior is generally considered the most immediate predictor of the actual execution of that behavior in the future, a substantial number of people do not act on these intentions (Sheeran, 2002). The objective of this study is to contribute to the emerging body of research that aims to bridge the gap between people's intentions and behaviors often found in behavioral determinant studies applying intention-behavior models. The present research was conducted within the framework of the Theory of Planned Behavior (Ajzen, 1991).

The Theory of Planned behavior (TPB; Ajzen, 1991) is one of the most frequently used theories to explain health behaviors (see for some reviews Albarracin et al., 2001; Armitage \& Conner, 2001; Godin \& Kok, 1996). The theory has been applied successfully to understand and predict behavior in a range of health domains, such as condom use (Albarracin et al., 2001), ecstasy use (Peters et al., 2008), dieting behavior (Armitage \& Conner, 1999), and exercise behavior (Biddle \& Nigg, 2000). The TPB, just like its predecessor the Theory of Reasoned Action (Fishbein \& Ajzen, 1975), considers a person's intention to perform a certain behavior as the most immediate predictor of actual execution of that behavior in the future. The intention-behavior relation can be subject to factors that hinder (i.e. barriers) or facilitate intention execution. The intention itself is predicted from attitude, i.e. the person's beliefs about the outcomes of the behavior and the importance of these outcomes; subjective norm, i.e. perceived norms of other people and the importance to comply with these references; and perceived behavioral control, similar to Bandura's concept of self-efficacy (Bandura, 1997), i.e. the degree to which the person feels in control over the target behavior. Besides affecting intention, perceived behavioral control can also to exercise a direct influence on behavior, especially when perceived control is an accurate assessment of someone's actual control over the behavior (Glanz et al., 2002).

The strength of the TPB is that it consistently predicts a substantial proportion of future behavior with only a few predictors. Intention accounts on average for about $30 \%$ of the variance in behavior in prospective studies (Sheeran, 2002). However, notwithstanding the model's efficiency, this leaves on average the gross of variance in future behavior unexplained. According to Sheeran (2002), the "limited" predictive value of someone's present intention for future behavior can be largely attributed to so-called "inclined abstainers", i.e. people with a positive intention towards the behavior but who fail to act accordingly. The discrepancy between intention and behavior is referred to as "the intention-behavior gap". The identification of factors that help close this gap has become a focal point of research (Sniehotta, Scholz, \& Schwarzer, 2005). Several studies have already shown that 
additional cognitive variables, the alignment between cognitive variables and intention, the stability of intention over time, or interactions with intention and other cognitive predictors can help bridge the intention-behavior gap (Godin et al., 2005; Norman et al., 2003; Sheeran \& Abraham, 2003; Sniehotta et al., 2005). The purpose of the present study is to contribute to this emerging field by investigating the role of so-called "instrumental activities" (Bagozzi, 1992).

Instrumental activities are core-constructs in Intervention Mapping. Intervention Mapping is a protocol for the systematic development of theory- and evidencebased health promotion interventions (Bartholomew et al., 2006). In the process of Intervention Mapping, researchers are encouraged to not only focus on improving the actual health behavior, but also on promoting instrumental activities (referred to as "performance objectives"). Within the IM framework, instrumental acts are behaviors that people can or should perform before the actual health behavior can occur. Instrumental acts can be preparatory behaviors (e.g. someone has to obtain and carry condoms, and negotiate condom use to increase the possibility of using condoms during sexual intercourse; Sheeran et al., 1999), but can also be functional from a theoretical viewpoint (i.e. self-regulatory activities to enhance control over goal enactment). Instrumental activities are temporally located between the formulation of an intention and the execution of the health behavior, i.e. it is only after someone intends to perform the health behavior that the person will consider engaging in functional sub-behaviors. This is also the case for the functional role of instrumental activities, since failure to always perform preparatory behaviors increases the probability that a person has to deal with barriers in order to execute the health behavior (or vice versa, i.e. engaging in instrumental acts facilitates the behavior). This has led to the idea that instrumental activities can help explain how people translate their intentions into behavior (Brian et al., 2002).

The present study was conducted among HIV-infected patients using Highly Active Antiretroviral Therapy (HAART) to suppress HIV-replication and thereby regain and maintain a healthy immune function. Just as with all medical treatments, the effectiveness of HAART depends on whether patients use the medication accurately. More than for other diseases, HAART demands high levels of adherence in order to remain effective over the years (i.e. $>90-95 \%$ of the medication should be taken correctly; Bangsberg et al., 2003; Gross et al., 2006; Howard et al., 2002; Liu et al., 2006; Paterson et al., 2000). Many HIV-infected patients do not achieve these levels of adherence and research has therefore focused on the identification of factors that predict (non)adherence to HAART (Ammassari et al., 2002; Fogarty et al., 2002; Godin et al., 2005; Simoni et al., 2006). However, until today few studies have used social psychological models to understand, predict, or enhance adherence to HAART (Coté \& Godin, 2005; Simoni et al., 2006). One purpose of the 
present study was therefore to employ the TPB to predict adherence among HIVinfected patients. Moreover, we identified three instrumental activities and investigated whether these helped close the intention-behavior gap.

The instrumental activities studied in the present research consist of two theory-derived activities and a preparatory behavior. First, the TPB clearly describes how people formulate their intentions (i.e. goals), but is less specific about what makes people successfully act upon their intention. In contrast, Self-Regulation Theory (SRT) does suggest how people may become more successful in achieving their goals. Zimmerman (Zimmerman, 2000) defines self-regulation as "selfgenerated thoughts, feelings and actions that are planned and cyclically adapted to the attainment of personal goals". Some of the core processes involved in behavioral self-regulation are self-monitoring of behavior to evaluate whether one's performance is up to the standard, and, if not, to develop and implement strategies to achieve the goals set (adaptive self-reactions; Zimmerman, 2000). Based on these principles, two instrumental acts were defined: "Self-monitoring of the accuracy of medication intake" and "When medication intake has been inaccurate, developing solutions to improve future intake". Besides these two self-regulatory activities, we included the preparatory behavior "Carry medication along when not at home". We assumed that people would especially engage in these activities if they were more motivated to perform the behavior, and that these activities are instrumental for translating the adherence intention into actual adherence. In other words, we assumed that these activities would mediate the intention-behavior relation.

When instrumental activities mediate the intention-behavior relationship, or otherwise contribute to the model as a moderator or predictor, interventions could also focus on promoting these activities. Knowledge of the relevant determinants for these behaviors is therefore also useful, especially since the most important determinants may differ between the health behavior and the instrumental activities (Van Empelen \& Kok, 2008). This study therefore aims to predict adherence to HAART using the Theory of Planned Behavior, to study whether the instrumental activities mediate the intention-behavior relation, and finally to explore which TPB determinants predict the relevant instrumental activities. 


\section{METHODS}

\section{Participants and procedures}

The present study was part of a larger trial that investigated the effectiveness of an intervention to enhance adherence to HAART (de Bruin et al., 2005; submitted). Patients visiting the Academic Medical Center in Amsterdam, the Netherlands, between September 2005 and January 2007 were asked to participate. Eligible patients were treatment-experienced ( $>6$ months on HAART), older than 18 years, Caucasian and Dutch-speaking, started antiretroviral treatment not earlier than 1996, were older than 18 years, had no known psychiatric disorders or drug or alcohol problems, and were able to comply with the study visiting schedule. HIVnurses and physicians approached eligible patients during routine visits and provided verbal and written information about the study. Patients were asked to consider participation for two weeks before making their final decision. The study was approved by the hospitals' ethics committee and all patients provided written informed consent. During this randomized controlled trial, all patients filled out questionnaires before and after the 3-month intervention period. Moreover, medication use was monitored with the so-called MEMS-cap (AARDEX, Itd.). The MEMS-cap is an electronic pill cap that registers date and time of the opening of the bottle, and is by many considered as the best measurement instrument for medication adherence (Farmer, 1999; Fennie et al., 2006; Paterson et al., 2002). The data provided by participants in the control group of this trial were used for the present analysis.

\section{Measures}

The pre- and post-intervention questionnaires (i.e. Time 1 and Time 2) contained self-reports of adherence, the self-regulatory activities "Self-Monitoring" and "Developing solutions", and the preparatory behavior "Carry medication". All these behaviors were assessed on a 5-point scale, asking participant to indicate whether they (1) Never to (5) Always engaged in the behavior.

For all four behaviors, i.e. adherence and the three instrumental activities, intentions, attitudes, perceptions of self-efficacy and perceived social norms were assessed. These were always framed specifically for each behavior according to the principle of compatibility (Ajzen, 1988; Fishbein \& Ajzen, 1975). Intentions were assessed with two items for each of the four behaviors, asking people 1. what they intend to always do, and 2. what they expect to always do. For example, "I intend to always consume my medication on time (within one hour of intended intake time, 
e.g. 9 a.m.), regardless of the context", or "I expect to always carry medication along when I leave my house". Response scales were (1) Definitely not to (5) Definitely and correlations ranged from .73 to .86 .

Attitudes were measured by asking people to rate what they thought of always conducting each behavior using three items: (1) Very unwise - (5) Very wise, (1) Very unimportant - (5) Very important, and (1) Very unpleasant - (5) Very pleasant. Cronbach's alphas increased significantly after deletion of the unpleasant-pleasant item (correlations of the two remaining items ranged from .61 to .71). Self-efficacy was assessed with a single item for each behavior with e.g. "Imagine you intend to always take your medication on time. Do you expect to always succeed in taking your medication on time, regardless of the context?". Response categories ranged from (1) Definitely not to (5) Definitely. The Subjective norms were assessed with two items. First, patients were asked what they thought important people in their environment think they should do. For example, "I think that the people who are important in my life find it (1) Absolutely not important to (5) Very important that 1 always take my medication on time, regardless of the context." In addition participants were asked whether they felt (1) Strongly hindered to (5) Strongly supported by people in their environment to always perform each specific behavior. Correlations ranged from .61-.67.

Mean scores were computed for each person on each determinant assessed with multiple items. In the analyses discussed in the Results section, we always used the determinants assessed specifically for the dependent variable in the respective analysis. Thus, in case adherence was the dependent variable, we used the attitude, self-efficacy, subjective norm and intention assessed specifically for adherence. When e.g. self-monitoring was the dependent variable, we used the TPB predictors assessed specifically for that behavior.

Participants were also asked to report at what time they intended to take their medication (e.g. 9 am and $9 \mathrm{pm}$ ). All the items that concerned adherence behavior were thereafter framed as "within 1 hour before and after your intended time" (e.g. I always intend to take my medication within 1 hour of my intended time). Although we also included an adherence self-report measure in the questionnaire, the dependent variable in this study was the objectively collected adherence data (MEMS-data). We used the Powerview software (AARDEX, Itd) to download these data and compute the percentage of doses that were taken within 1 hour of each patients intended time. Mean scores were computed over the 28 days before completion of the post-intervention questionnaire (Time 2). Finally, we collected background variables and demographic characteristics of the study sample. 


\section{RESULTS}

Within the timeframe set for this study, 67 patients were randomized to the control condition and 63 completed the baseline questionnaire. Of these, 57 also completed the post-intervention questionnaire, and 58 provided MEMS data at Time 2. Five of these 58 participants showed inaccurate MEMS-use and these MEMS-data were therefore recoded to missing. The participants $(N=63)$ were predominantly male $(89 \%)$, had a mean age of 49.7 years $(S D=9.7)$, the majority was employed $(70 \%)$, was medium (33\%) to highly educated $(35 \%)$, and used a once-daily antiretroviral regimen (56\%).

To explore the relations between the adherence TPB predictors at Time 1 and adherence behavior at Time 2, we first examined the correlations (see Table 1). Selfefficacy, attitude and subjective norms towards adherence correlated with patients' intention to adhere to the treatment, and only self-efficacy and intention predicted adherence at Time 2. Table 1 also shows the correlations between the three instrumental activities and intention at Time 1 , and adherence behavior at Time 2 . Note that the instrumental activities "Carry medication", "Self-monitoring" and "Developing solutions" were all related to intention, while only Carry medication and Self-monitoring predicted adherence at Time 2. The treatment-experienced patients were, as one may presume, generally highly motivated, confident and adherent.

\section{Prediction of adherence}

Figure 1 represents the proposed causal model. To test this model, we first regressed adherence at Time 2 on the full TPB model (Time 1). In a two-step linear regression patients' self-efficacy, attitude and subjective norm regarding adherence were entered in a first step, and the adherence intention was entered in a second step. Of the proximal determinants, only self-efficacy predicted adherence $(p<.01)$. When intention entered the model in a second step, the effects of self-efficacy became non-significant and intention was the strongest predictor of behavior $(p<$ .05). The four predictors combined explained $37 \%$ of the variance in adherence at Time 2 (see Table 2, model 1). 
Table 1. Means, standard deviations and intercorrelations for study variables at baseline (Time 1) and adherence at Time 2

\begin{tabular}{|c|c|c|c|c|c|c|c|c|}
\hline & 1 & 2 & 3 & 4 & 5 & 6 & 7 & 8 \\
\hline 1 Adherence & 1 & $0,69 * * * *$ & $0,34^{*}$ & 0,18 & $0,56^{* * * *}$ & 0,26 & $0,54^{* * * * *}$ & 0,23 \\
\hline 2 Carry medication & & 1 & $0,42^{* * *}$ & $0,29^{*}$ & $0,64^{* * * *}$ & & & \\
\hline 3 Self-monitoring & & & 1 & $0,30^{*}$ & $0,45^{* * *}$ & & & \\
\hline 4 Develop solutions & & & & 1 & $0,41^{* * *}$ & & & \\
\hline 5 Intention & & & & & 1 & $0,63^{* * * * *}$ & $0,72^{* * * *}$ & $0,41^{* * *}$ \\
\hline 6 Attitude & & & & & & 1 & $0,40^{* * *}$ & $0,55^{* * * *}$ \\
\hline 7 Self-efficacy & & & & & & & 1 & 0,20 \\
\hline 8 Norm imp. others & & & & & & & & 1 \\
\hline Mean & 84.44 & 4.31 & 4.58 & 3.98 & 4.57 & 4.78 & 4.20 & 4.43 \\
\hline SD & 19.42 & 1.08 & 0.76 & 1.30 & 0.69 & 0.39 & 0.92 & 0.71 \\
\hline
\end{tabular}

In a second linear regression analysis we examined whether any of the three instrumental activities assessed at Time 1 improved prediction of adherence at Time 2 given the TPB predictors in the model. Considering the modest sample size, only the significant predictors were retained in the analysis. Thus, in the first step the adherence intention was entered in the model and in the second step the three instrumental activities were added. Developing solutions was not significant and therefore deleted from the model. Both Self-monitoring and Carry medication were significant in each other's presence ( $p<.02$ and $p<.01$ respectively) and together explained an additional $25 \%$ of variance in behavior on top of the $32 \%$ explained by the adherence intention. The intention was no longer significant in the presence of the two significant mediators ( $p>50$; see Table 2 , model 2 ). When these analyses were repeated including all the TPB predictors, the results were essentially similar.

We conducted additional analyses to examine whether the observed relations were not confounded by background variables. We therefore repeated the analyses entering gender, educational level, age, and employment status in a first step in the model. However, none of the demographic variables predicted adherence (all $p$ values $>.2$ ). We also repeated each analysis including past behavior (i.e. mean MEMS adherence over 4 weeks at Time 1), which correlated highly with adherence at Time $2(r=.82, p<.01)$. Despite that including past behavior as a covariate in the analyses substantially reduced the proportion of variance in adherence (Time 2) to be explained by the TPB predictors, predictors that were significant in a given model in Table 2 remained significant after adjustment for adherence at Time 1. 


\section{CHAPTER 5}

\section{Mediation of the intention-behavior relation}

Mediation of the relation between two variables occurs when the total effect between an independent and a dependent variable is, at least partially, accounted for by a third variable (i.e. the mediator) that is affected by the independent variable and affects the dependent variable. According to Mackinnon and colleagues (2002), the joint significance test is the best method to examine such mediation effects. Figure 1 shows that there are two mediating variables in the model, i.e. intention mediates the self-efficacy-adherence relation, and the instrumental activities mediate the intention-behavior relation. Basically, for intention to mediate the self-efficacy-adherence relation, 1) self-efficacy has to predict intention, and 2) intention has to predict adherence controlling for self-efficacy. For the instrumental activities to mediate the intention-behavior relation, 1) intention has to predict the instrumental activities, and 2) the instrumental activities have to predict adherence, controlling for intention. Since there are multiple potential mediators in the present analyses, each instrumental act has to significantly predict adherence, controlling not only for intention, but also for the other instrumental activity.

Table 2. Summary of hierarchical regression analyses $(n=50)$. Standardized betas and R-square values are shown for Model 1: Adherence regressed on TPB, Model 2: Adherence regressed on intention and the significant instrumental activities.

\begin{tabular}{|c|c|c|c|c|c|}
\hline \multirow[b]{2}{*}{ Steps } & \multirow[b]{2}{*}{ Variables entered } & \multicolumn{2}{|c|}{ Model 1} & \multicolumn{2}{|c|}{ Model 2} \\
\hline & & Beta & Beta & Beta & Beta \\
\hline \multirow[t]{3}{*}{ 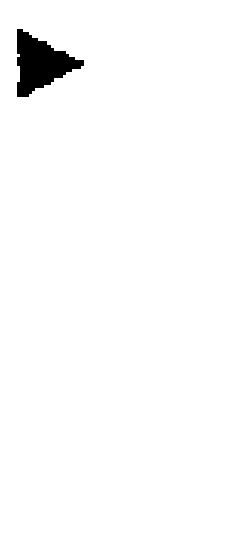 } & Self-efficacy & $0.54 * *$ & 0,29 & & \\
\hline & Attitude & -0.05 & -0.19 & & \\
\hline & Subjective Norm & 0.13 & 0.07 & & \\
\hline \multirow{3}{*}{$\begin{array}{l}- \\
V\end{array}$} & Intention & & $0.44^{*}$ & $0.57 * *$ & 0.09 \\
\hline & Carry medication & & & & $0.51 * *$ \\
\hline & Self-monitoring & & & & $0,30^{*}$ \\
\hline$R^{2}$ & & $0,31 * *$ & 0,37 & $0,32 * *$ & 0,57 \\
\hline$\Delta R^{2}$ & & & $0,06^{*}$ & & $0,25 * *$ \\
\hline
\end{tabular}

${ }^{*} p<.05 ; * *<.01$. Note: due to missing values on some variables, Model $1: n=50$, Model $2: n=48$ 
The regression analyses in Table 2 show that the second condition for each mediation analysis has been met, even after controlling for demographics, the nonsignificant TPB predictors, and past behavior. The correlations reported in Table 1 suggest that also the first condition for mediation has been met, i.e. self-efficacy predicts intention and intention predicts the instrumental activities. We conducted additional regression analyses to also rule out that these correlations were confounded by demographics, the other TPB variables or past behavior. In view of the skewed distribution of the dependent variables (i.e. intention, Self-monitoring and Carry medication), these were dichotomized. We first regressed the adherence intention on self-efficacy, subjective norm and attitude towards adherence. The logistic regression analysis showed that only self-efficacy predicted the adherence intention $(p<.01)$, also after controlling for demographics and past behavior. Moreover, the logistic regression analyses showed that the relation between the adherence intention and instrumental activities was not confounded.

As an alternative to the approach of Mackinnon, Preacher \& Hayes (2004; 2008) have developed a non-parametric bootstrapping test to investigate the significance of medication effects. This test does not make assumption about the distribution of variables and the test statistic, and can be applied to smaller samples with more confidence. We therefore re-examined the primary mediation analyses, i.e. intention-behavior mediation, with the Preacher and Hayes test (2008). Using a $95 \%$ confidence interval and the recommended 5000 bootstraps, this test yielded the same outcomes as the Joint Significance test, i.e. that patients with higher adherence intentions are more likely to both Self-monitor and Carry medication along, and that both these activities are functional for the translation of the adherence intention into actual adherence.

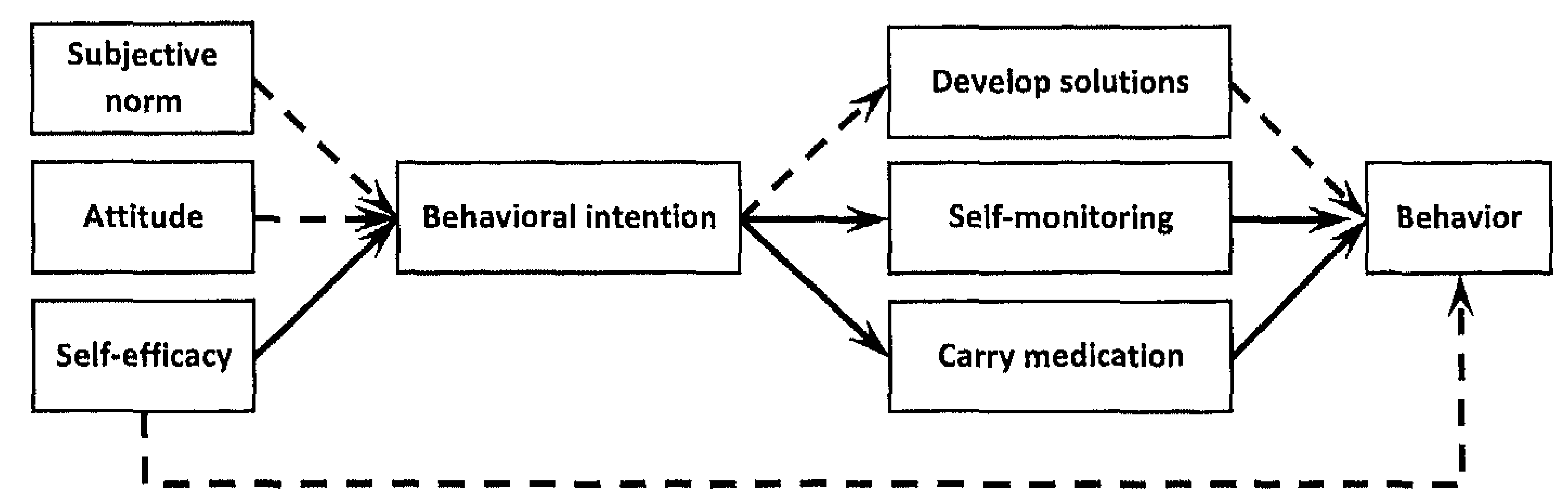

Figure 1. A graphic representation of the Theory of Planned behavior including the mediating variables. The solid lines present relations between the predictors and adherence behavior that were supported by the data, the dashed lines present presumed relations that were not found. 


\section{Determinants of the relevant instrumental activities}

According to the adherence regression analyses, HIV-infected patients who selfmonitor their medication intake and who carry medication along when they are not at home, are more likely to accurately take their medication. Hence, intervention programs could also employ strategies to promote these instrumental activities and thereby more effectively change adherence. To gain insight in the factors that differentiate the patients who Self-monitor or Carry medication from those who do not, we explored which TPB determinants, assessed specifically for each instrumental activity at Time 1, predicted Self-monitoring and Carry medication assessed at Time 2. To prevent confusion, note that the TPB determinants in the preceding analyses referred to adherence itself, not to the instrumental activities. Moreover, we limited these secondary, exploratory analyses to a brief examination of the correlation structure only.

The univariate analyses showed that for both instrumental activities attitude, self-efficacy and subjective norm correlated with intention, and that all TPB variables predicted self-reported behavior at Time 2 . The specific intentions were the strongest predictors of the instrumental activities (see Table 3). The observed correlation patterns suggest that also Self-monitoring and Carrying medication are primarily predicted by intentions. Moreover, patients with more positive attitudes, higher levels of self-efficacy, and who experience a supportive social norm, have more positive intentions and are more likely to engage in the instrumental activities.

\section{DISCUSSION}

The primary objective of this longitudinal study was to contribute to the growing body of research that seeks to identify factors that bridge the gap between peoples' intention and behavior. Whereas previous studies with this aim have focused on the role of (additional) cognitive variables (Godin et al., 2005; Norman et al., 2003; Sheeran \& Abraham, 2003; Sniehotta et al., 2005), the present study investigated whether so-called instrumental activities can help explain why people are (un)successful in acting upon their intentions. The study was conducted in the domain of adherence to HAART among HIV-infected patients, a field that has remained relatively a-theoretical despite a vast number of publications on predictors of (non)adherence (Coté \& Godin, 2005; Simoni et al., 2006). A secondary aim was therefore to investigate whether the TPB model could be used to effectively predict adherence. 
Table 3. Mean, standard deviations and intercorrelations for Self-monitoring and Carrying medication with each behaviors' specific TPB predictors

\begin{tabular}{|c|c|c|c|c|c|}
\hline & 1 & 2 & 3 & 4 & 5 \\
\hline 1 Carry medication & 1 & $0,62 * * * *$ & $0,32^{*}$ & $0,52 * * * *$ & $0,28 *$ \\
\hline 2 intention & & 1 & $0,53^{* * * *}$ & $0,56 * * * *$ & $0,37^{* *}$ \\
\hline 3 Attitude & & & 1 & $0,46 * * * *$ & $0,36^{* *}$ \\
\hline 4 Self-efficacy & & & & 1 & 0,13 \\
\hline 5 Norm important others & & & & & 1 \\
\hline Mean & 4.3 & 4.64 & 4.79 & 4.35 & 4.35 \\
\hline $\mathrm{SD}$ & 1,01 & $\underline{\underline{0,52}}$ & 0,39 & 0,96 & 0,76 \\
\hline 1 Self monitoring & 1 & $0,67 * * * *$ & $0,54 * * * *$ & $0,37 * * *$ & $0,38^{* * *}$ \\
\hline 2 Intention & & 1 & $0,61 * * * *$ & $0,51 * * * *$ & $0,44 * * * *$ \\
\hline 3 Attitude & & & 1 & $0,42^{* * *}$ & $0,57 * * * *$ \\
\hline 4 Self-efficacy & & & & 1 & $0,29 *$ \\
\hline 5 Norm important others & & & & & 1 \\
\hline Mean & 4.48 & 4.52 & 4.57 & 4.36 & 4.2 \\
\hline SD & 0.71 & 0.82 & 0.63 & 0.85 & 0.78 \\
\hline
\end{tabular}

p-value*<.05; ${ }^{* *}<.01 ; * * *<.005 ; *<.001$. Note: only including patients with data on

dependent variable; pairwise deletion of missing values, $n=53-56$.

Validation of the TPB model in the domain of HAART adherence was limited by the small sample size in the study. Whereas the adherence attitude, subjective norm and self-efficacy correlated with the adherence intention as expected, only self-efficacy predicted intention in the regression analysis. Although this may imply that people's attitudes and subjective norms do not explain why some patients have high and others have lower adherence intentions, the absence of a significant finding may also be caused by a lack of power to detect this relation. The same reasoning applies to the self-efficacy-adherence relation, which became nonsignificant after intention was added to the model (i.e. full mediation). This finding may imply that either patients' self-efficacy was not an accurate assessment of their actual control over the behavior (Glanz et al., 2002), or that the analysis lacked power to find an existing relation. Notwithstanding these non-significant relations, the parsimonious TPB explained $37 \%$ in adherence measured objectively with the MEMS-caps over a 1-month period. Moreover, consistent with longitudinal TPB studies in other domains, the adherence intention was the strongest predictor of adherence and accounted for approximately $30 \%$ of the variance in behavior (Sheeran, 2002). Finally, the additional analyses in which we controlled for demographics and past behavior provide tentative evidence that the significant 


\section{CHAPTER 5}

relations were causal. Thus, despite its limitations, this study suggests that the Theory of Planned behavior is a useful framework for understanding and predicting treatment adherence among HIV-infected patients using HAART.

In line with the theoretical model, the strongest predictor of adherence was the adherence intention. Most importantly, the postulation that instrumental activities would mediate the intention-behavior relation was confirmed. Two out of three mediating variables simultaneously predicted adherence, fully mediated the intention-behavior relation, and explained a considerable proportion of variance in adherence in the presence of the TPB predictors. These instrumental activities thus explain how these patients translated their adherence intentions into actual behavior. Moreover, the results suggest that adherence interventions may be more effective in case they also focus on promoting Self-monitoring and the Carrying of medication. The observation that attitudes, self-efficacy and subjective norms (Time 1) towards Self-monitoring and Carrying medication correlated strongly with the intentions (Time 1) to Self-monitor and Carry medication, and with patients' selfreports of these instrumental acts (Time 2), provides some preliminary evidence for designing the content of such interventions.

One instrumental activity, i.e. Developing solutions, did not mediate the intention-behavior relation. Although this activity correlated with the adherence intention, it did not predict behavior in the presence of intention and the other instrumental acts. One would expect however that adaptive self-reactions, the activity we intended to measure with this item, are more proximate to adherence behavior than self-monitoring. Possible explanations for the lack of an effect of Developing solutions are that the item did not accurately measure adaptive selfreactions, that the construct was valid but that patients' coping strategies were generally ineffective, or that patients failed to implement effective solutions in their daily life. Both these latter explanations would be relevant for the development of future adherence interventions, but the present research does not permit any conclusive remarks on these topics.

The present study is not the first to investigate whether instrumental activities mediate the intention-behavior relation. As mentioned in the introduction, Brian, Fisher, and Fisher (2002) addressed this topic previously. They found that preparatory behaviors regarding condom use (i.e. obtain condoms, carry condoms and negotiate condom use) mediated the intention-behavior relation. Although our study rationale is largely similar to Brian's, there are some differences worth mentioning. In the first place, both studies used data from two time points. Whereas Brian and colleagues choose for a time lag between intention and the mediators (i.e. intention Time 1 and the mediators and the dependent from Time 2), we decided to have a time lag between the mediators and the dependent. Both 
designs are probably suboptimal, since ideally the TPB predictors are assessed at Time 1, the mediators at Time 2 and the dependent at Time 3 (see for example Sniehotta at al., 2005). Secondly, while in both studies the intentions (as well as the other TPB predictors) were assessed specifically for the health behaviors and three instrumental activities, Brian and colleagues (2002) computed the mean Condom use intention based on the intentions towards all four behaviors. This may have strengthened the correlation between intention and the mediating variable (i.e. the mean score of the three preparatory behaviors). Instead, we judged it to be more appropriate to use the health behavior-specific intention (i.e., the adherence intention). Finally, Brian and colleagues used two datasets from a large number of participants, while our study sample was relatively small. This decreased the power of the statistical tests and, although the present sample was a random selection from a larger group of patients (i.e. 66/133 participants were randomized to the control group), presumably limited the representativeness of our study sample for the general population. We therefore recommend tentative interpretation of the study outcomes.

Despite the limitations of the present study, the findings suggest that the Theory of Planned behavior may be useful model for predicting treatment adherence among HIV-infected patients using HAART. Moreover, the mediation analyses showed that instrumental activities can indeed help to bridge the gap between intentions and behaviors. Future studies in other domains should demonstrate whether instrumental activities consistently predict health behavior, and whether this occurs through mediation of the intention-behavior relation. If so, instrumental activities could be a valuable addition to existing behavioral theories, thereby enhancing the understanding of people's health behaviors and guiding the content of behavior change interventions. 


\section{CHAPTER 6}

How adherence care impacts treatment outcomes in HIV: A meta-analysis of randomized controlled trials

Marijn de Bruin, Wolfgang Viechtbauer, Herman P Schaalma, Gerjo Kok, Charles Abraham, Harm J Hospers

Submitted 


\section{CHAPTER 6}

\section{ABSTRACT}

Context: Poor medication adherence to highly active antiretroviral therapy (HAART) limits HIV treatment effectiveness. Systematic reviews examining the content of interventions to improve adherence can identify practical and effective interventions. Since the quality of standard care provided to control groups in HAART adherence trials varies between studies, meta-analyses should control for this variability to produce more accurate estimates of intervention effects.

Objective: To examine whether viral load and adherence rate differences can be accurately explained by the quality of interventions designed to enhance HAART adherence when controlling for variability of care delivered to the control groups. Data sources: Embase, PsycINFO, and Medline were searched for randomized controlled trials (RCTs) of HAART adherence interventions published between January 1996 and January 2009.

Study selection: 1342 records were retrieved and 52 articles were examined in detail. Two coders determined which studies described RCTs of HAART adherence interventions. Studies of addicted patients, patients $<18$ years, HIV-care in developing countries, or non-autonomous behavior were excluded, yielding a final sample of 34 trials.

Data extraction: Two coders independently examined the content of care delivered to intervention and control groups as well as study details.

Results: The quality of experimental interventions, defined as the number of theoryand evidence-based behavior change techniques targeting adherence, accurately explained intervention effects after controlling for variability in standard care provided to active controls. Although interventions were generally beneficial, their effectiveness was noticeably reduced with increasing levels of standard care.

Conclusions: Patients in intervention and control groups were exposed to effective adherence care. Clinical practice may be best served by adoption of current best practice, which is expected to increase viral load suppression rates among patients in developed countries by $14-21 \%$ points. Future meta-analyses of (behavior change) interventions should control for variability in care delivered to active controls. 


\section{INTRODUCTION}

HIV can be effectively suppressed with a combination of medicines referred to as highly active antiretroviral therapy (HAART), but approximately $50 \%$ of patients do not achieve and sustain the high levels of medication adherence required for optimal viral suppression (i.e., 90-95\%; Gross et al., 2006; Knobel et al., 2002; Paterson et al., 2000; Sethi et al., 2003). Numerous adherence supporting interventions have been developed and evaluated, and systematic reviews and metaanalyses have synthesized these studies up to 2006 (Amico et al., 2006; Haddad et al., 2000; Rueda et al., 2006; Simoni, et al., 2006). While several interventions have been found to be effective, reviews have not clarified why some interventions are more effective than others. Hence, guidance for improvement of health care is limited.

Systematic reviews and meta-analyses of behavior change intervention trials usually examine the content of interventions (or treatments) in some detail, but rarely explore the content of care provided to active control groups. This is problematic since, as evidence suggests, the content and effectiveness of "standard" or "usual" care provided to control groups can vary considerable between studies (de Bruin et al., in press; Wagner \& Kanouse, 2003). Intervention effects are then best understood by examining the difference in care provided to both groups. Without such differential content analyses it is unclear why similar experimental interventions generate different effect sizes at different study sites (i.e., larger in settings with minimal compared to advanced standard care). Hence, meta-analyses of behavior change interventions must consider the content of care provided to both intervention and active controls to accurately characterize the added value of interventions, and so understand what intervention content is associated with increased effectiveness.

\section{Defining the active content of experimental and standard care}

The active content of behavior change interventions consists of theory- and evidence-based behavior change techniques (BCTs; Abraham \& Michie, 2008; Bartholomew et al., 2006; de Bruin et al., in press) Well-designed behavior change interventions contain carefully-chosen BCTs that aim to change important determinants of the target behavior. For example, if patients require certain skills, guided practice and modeling are likely to be effective BCTs. The application of the selected $\mathrm{BCT}$ s should change the corresponding behavioral determinants, consequently behavior, and ultimately improve health (Bartholomew et al., 2006). The number of 
relevant BCTs applied in an intervention can be considered the intervention dosage. Since this dosage represents the degree to which the intervention deals with relevant behavioral problems using evidence-based $\mathrm{BCTs}$, we will refer to this as the "intervention quality".

Just as the intervention, standard care can contain effective BCTs. We recently assessed the content of care provided to active control groups in HAART-adherence intervention trials and found that it contained numerous $B C T$ s targeting important determinants of adherence. These BCTs overlap with those often included in adherence intervention reports (e.g., providing verbal and written information, developing tailored medication intake schedules, supplying reminders etc.). When we counted the number of techniques applied (i.e., "standard care quality"; SCQ), we found considerable variability in SCQ between studies, which in turn was strongly related to the proportion of patients that achieved an undetectable viral load (de Bruin et al., in press). These findings demonstrated that the control groups - reportedly all receiving "care as usual" - were actually exposed to widely varying forms of effective adherence care. This variability should be taken into account when interpreting and comparing the effects of experimental treatments. Although several meta-analyses have shown that the type of control group (e.g., active versus passive; placebo versus "care as usual") can account for variance in effect sizes (Acarturk et al., 2009; Prendergast et al., 2002), including a measure that captures variability in care provided to active controls has to our knowledge not been done before.

\section{Study objectives}

The aim of the present meta-analysis was to examine whether the outcomes reported by RCTs of HAART adherence interventions could be accurately explained by the quality of the interventions, while controlling for variability in SCQ between studies. With that goal in mind, we first examined whether intervention and SCQ could accurately predict the treatment success, and then whether the difference in quality between the experimental treatment and standard care accurately predicted the effect sizes. We controlled for potential confounders and moderators such as methodological or population differences. 


\section{METHODS}

\section{Literature search and selection procedure}

Embase, PsycINFO, and Medline were searched until from January 1996 up to January 2009 for articles evaluating behavior change interventions to enhance adherence to HAART. Search terms were: (adherence or compliance) AND (HIV or AIDS) AND (random*) in "All Text". In total 1342 records were examined (including 454 duplicates). References in obtained articles and in relevant reviews were also searched. Interventions focusing specifically on patients facing challenges essentially different from those of the main study sample were excluded. These included drug or alcohol addicted patients, patients younger than 18 years old, and those living in developing countries. In addition, directly observed therapy (DOT) interventions were excluded since these do not focus on autonomous patient behavior. Figure 1 depicts the process of study inclusion and exclusion.

Figure 1. Flowchart: Inclusion and exclusion of studies

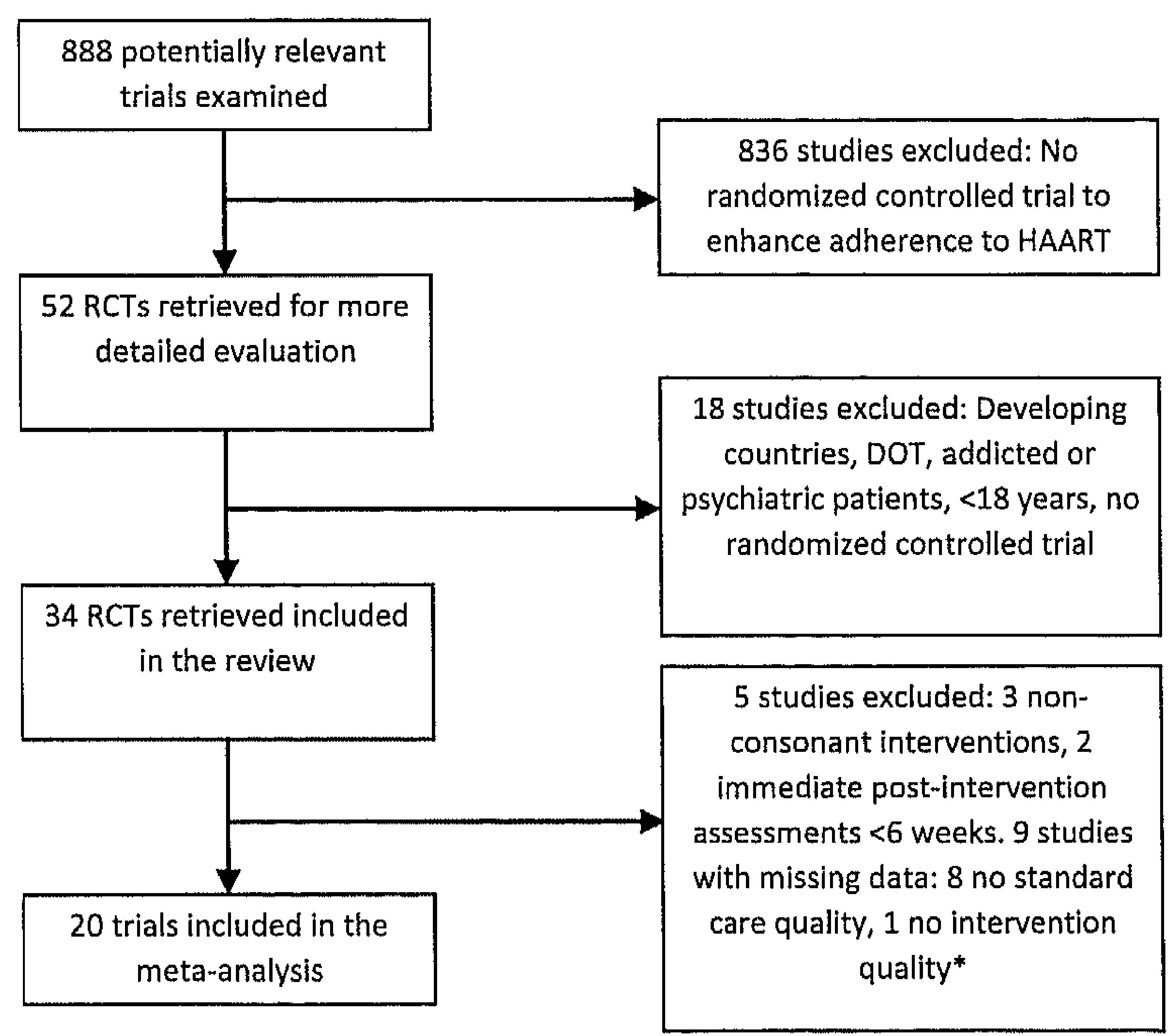

*Note: Only primary reasons for exclusion or dropout are reported for those studies not retained in either the adherence or viral load analysis 


\section{Assessment of intervention and standard care quality}

To allow coding of intervention and standard care, authors were asked to provide intervention protocols and to complete a standard care checklist. Two coders (authors GK and HPS) independently coded the standard care and intervention materials using a 41. tem taxonomy of BCTs targeting important determinants of adherence behavior (de Bruin at al, in press), an adapted version of the 26 item taxonomy developed by Abraham \& Michie (2008). Coders were blind to the author and journal details and the results and discussion sections. Results were discussed under supervision of the first author and disagreements were resolved through discussion.

Coding results were used to compute SCQ and intervention quality scores based on three rules. First, BCTs that were applied only once or only at the beginning of the intervention were given 1 point. Second, BCTs that were tailored to individual patients or required their active participation (e.g., instead of top-down instructions) were given 2 points (Brug et al., 1998; Mullen et al., 1985; Noar et al., 2007; Petty et al, 2002; Prochaska et al, 1997). Third, BCTs applied repeatedly during follow-up sessions were allocated a score of 2 . Thus, for example, a tailored technique applied repeatedly during follow-up was allocated 4 points. This weighting provided an index of strength in terms of personal relevance or tailoring, and of predominance or repetition. Treatment quality in intervention and standard care groups was computed by adding points across the included BCTs.

\section{Assessment of the outcome data and other study details}

Two coders (authors $M d B$ and $W V$ ) independently coded the details of study population, methods, outcome data, and the relative contact intensity of the intervention compared to standard care (1=the same in both groups, $2=$ more, and $3=$ much more in the intervention group; Kappa $=.82$ ). We collected intent-to-treat outcome data (viral load undetectable vs. detectable and adherence $\geq 95 \%$ vs. $<95 \%$ ) at the immediate post intervention assessment (IPIA), including those participants actually providing data (available cases). All authors of included studies were asked to confirm these coding results and to complete missing study details.

A priori, we formulated two criteria for including outcome data in the metaanalysis. First, we assumed a time-lag between exposure to an effective intervention and a non-adherent person achieving adherence levels of $295 \%$ (DiClemente \& Prochaska, 1998; Prochaska \& DiClemente, 1983). We also assumed a time-lag between improvements in adherence and the effects of improved 
adherence on viral load (i.e., shifting from detectable to undetectable). Since 12 weeks was suggested as a relevant cut-off for effectiveness in a previous review, we included studies in the viral load analyses in case the IPIA was $\geq 12$ weeks and in the adherence analyses if the IPIA was at least half that time ( $\geq 6$ weeks; Rueda et al., 2006). This rule excluded two brief study from the adherence analyses and three additional studies from the viral load analyses (Murphy et al., 2002; Remien et al., 2005; Safren et al., 2001; Wagner et al., 2006).

\section{Analyses}

For each trial, the proportion of intervention and control patients with an undetectable viral load or adherence $\geq 95 \%$ (the "success rate") were computed. To validate the separate intervention and SCQ scores, we first examined whether intervention quality and SCQ predicted the success rates in their respective groups. Several potential confounders were included to control for between-study differentces: 1 . inclusion of patients continuing treatment vs. patients starting (a new) treatment, 2. dominant ethnicity (Caucasian vs. Non-Caucasian), 3. no selection vs. selection of patients with treatment problems at baseline (detectable viral loads or adherence problems), 4. mean study year (e.g., 2001 for a study conducted between 2000 and 2002), 5. dropout percentage, and either 6 . detection threshold of the viral load assessments ( $<400 \mathrm{vs.} 400$ copies $/ \mathrm{mL}$ ) or 6 . adherence measurement by means of a bottle cap with embedded microchip to record bottle openings (MEMS-caps) versus self-reports. We expected success rates to be higher on average for Caucasian samples (de Bruin et al., in press; Golin et al., 2002; Nellen et al., 2004), without selection on baseline treatment problems (Amico et al., 2006), in more recent studies (due to improvements in medical regimes over time), when using a detection threshold of 400 copies/mL, and for self-reports (Liu et al., 2001; Melbourne et al., 1999; Paterson et al., 2002). We expected higher viral load success rates for treatment-experienced patients, but had no hypothesis regarding the direction of effect for dropout rates.

In a second step, we computed the rate differences for viral load and adherence by subtracting the success rate in the control group from the success rate in the intervention group. The difference score between SCQ and intervention quality was used to predict these rate differences. Due to randomization, the influence of between-study differences (e.g., confounders mentioned above) should be cancelled out. We therefore controlled these analyses for post-randomization within-study differences only, namely the relative contact intensity and the differential drop-out between conditions (Amico et al., 2008). 
All analyses were based on mixed-effects meta-regression models, using restricted maximum likelihood to estimate residual heterogeneity. In the success rates analyses, we emploved a bivariate mixed-effects model, allowing the true rates of the intervention and control group from the same study to be correlated (van Houwelingen et al., 2002). The intercept and slope of control and intervention groups were allowed to differ by including a dummy variable indicating the group and its interaction with the quality score in the model. The predictive power of all models is illustrated by calculating a (pseudo) $R^{2}$ statistic (Willett \& Singer, 1988).

\section{RESULTS}

Thirty-four RCTs were included in the review (Andrade et al, 2005; Berger et al., 2008; Collier et al., 2005; Dilorio et al., 2003, 2008; Golin et al., 2006; Goujard et al., 2003; Holzemer et al., 2006; Javanbakht et al., 2006; Johnson et al., 2007; Jones et al., 2003, 2007; Knobel et al., 1999; Koenig et al., 2008; Mann, 2001; Mannheimer et al., 2006; Milam et al., 2005; Murphy et al., 2002, 2007; Pradier et al., 2003; Rathbun et al., 2005; Rawlings et al., 2003; Remien et al., 2005; Reynolds et al., 2008; Rigsby et al., 2000; Safren et al., 2001, 2003; Simoni et al., 2007; Smith et al., 2003; Tuldra et al., 2000; Van Servellen et al., 2005; Wagner et al., 2006; Weber et al., 2004; Wohl et al., 2006). Thirty-three (co)authors could be contacted and provided additional study details. Most (27) studies were conducted in the United States, 21 focused on treatment-experienced patients, and 11 studies selected patients with treatment problems. The main ethnicity in 26 studies was nonCaucasian (i.e. African-American and Latino-Hispanic). Half the studies reported MEMS-cap data, the other half self-reported adherence. IPIA data for adherence could be obtained from 27 studies, and for viral load data from 26 studies. Six studies did not assess viral load and two authors did not provide these data on request. For further study details see supplementary material, Table S1.

\section{Intervention and standard care quality}

Twenty-two authors completed the standard care checklist and once SCQ could be coded from the article. Two authors did not respond to our request for these details and 9 could not provide this information. Intervention content could be coded for 28 studies. Three intervention descriptions were insufficient to allow content coding. Three more were coded as "non-consonant" (Mullen et al., 1985), because 
the intervention content did not focus on adherence (e.g., a general stress reduction intervention with no mention of adherence). Inter-coder reliabilities were good (mean $(\mathrm{sd})=0.75(.17))$. Standard care quality scores ranged from $2-28$ points $($ mean $(s d)=14.21(7.68))$. The quality scores computed from the intervention manuals ("manual quality") ranged from 4-36 points (mean (sd)=18.07 (8.62)).

Table 1 illustrates the coding results for one study (only coded BCTs are shown). Note that the "manual quality" does not accurately describe the difference between intervention and standard care. Out of the 16 BCTs coded in the intervention manual, $6(38 \%)$ were also part of standard care. Examining the set of studies for which both intervention and standard care quality were available, this overlap ranged from $0-50 \%$ (mean $(\mathrm{sd})=27 \%(15 \%)$ ). We expected that increasing levels of standard care would decrease the opportunity for interventions to introduce new techniques. In other words, as standard care quality increases, the percentage of overlapping techniques should increase as well. This hypothesis was supported by the data $(r=.68, p<.001)$.

The example in Table 1 also shows that standard care included techniques that were not described in the intervention manual. We therefore assessed whether the intervention groups received experimental care in addition to standard care. Since this was the case for all studies, the total range of BCTs provided to intervention participants were those applied in both intervention and standard care.

For the reasons stated above, we computed a "total intervention quality" score, comprising the total range of BCTs to which the intervention groups were exposed. This score should predict the success rates in the intervention groups. Since we expected that the difference between standard care and the experimental intervention would be the best predictor of the rate differences (i.e., rather than the uncorrected manual quality), we also computed a "unique intervention quality" score (see Table S1 for quality scores).

\section{Predicting the success rates in the intervention and control groups}

We first examined whether quality (i.e., total intervention quality for intervention group and standard care quality for control group) predicted viral load and adherence success rates in the respective groups, while controlling for several confounders. Neither the slope ( $p=.46$ for viral load; $p=.12$ for adherence) nor the intercept ( $p=.80$ for viral load; $p=.27$ for adherence) of the regression lines were significantly different for intervention versus control groups, which suggests that the standard care and intervention quality scores reflect the same underlying 
"quality" dimension. Results for the models assuming an identical intercept and slope are provided below.

- The quality score predicted viral load $(p<.01)$ and adherence $(p<.001)$ success rates, confirming that these quality measures for the content of care predict clinical and behavioral outcomes in both the intervention and control conditions. In addition, a number of confounders explained heterogeneity in the success rates. In the viral load analysis, non-Caucasian samples had a success rate that was on average .27 points lower than that of Caucasian samples $(p<.001)$. However, this difference was not found in the adherence analyses (.05 difference, $p=.48)$. Moreover, a viral load detection threshold of 400 copies $/ \mathrm{mL}$ yielded success rates that were on average .16 points higher than those based on stricter thresholds $(p=$ .04). Other potential confounders did not reach significance in the viral load analyses. For adherence, however, patients continuing treatment had on average a .17 points lower success rate in comparison to patients starting (a new) treatment $(p<.01)$. The selection of patients with a detectable viral load or adherence problems yielded a .16 points lower average success rate $(p=.03)$. The average success rate for adherence was also estimated to decrease by .004 points $(p=.03)$ for each percentage point increase in the dropout rate, suggesting that adherent patients were more likely to drop out than non-adherent patients. Finally, success rates based on self-reported adherence were on average .48 points higher than those based on MEMS-caps $(p<.001)$, and mean study year showed a trend towards being significant $(p=.09)$. Results are shown in Table 2 .

Overall, the models for viral load and adherence were highly predictive of the success rates $\left(R^{2}=.72\right.$ and $R^{2}=.82$, respectively). Removing non-significant predicttors had almost no effect on these results $\left(R^{2}=.64\right.$ and $\left.R^{2}=.79\right)$.

Figure 2 displays the proportion of patients with an undetectable viral load and $\geq 95 \%$ adherence as a function of quality after adjustment for confounders (e.g., for viral load adding .27 points to the observed success rates for studies with nonCaucasian samples, subtracting .16 points for a detection threshold of 400 copies $/ \mathrm{mL}$, etc.). The absence of ceiling effects suggests that the BCTs added by the interventions were effective regardless of the level of standard care. Moreover, the plots show that a number of control groups received standard care superior in quality and effectiveness to the total intervention care (i.e., standard care plus the experimental intervention) provided to a number of intervention groups. 
Table 1. Example of the quality coding results for one study.

\begin{tabular}{|c|c|c|c|c|}
\hline Determinant & Behavior change techniques & $\begin{array}{l}\text { Intervention } \\
\text { manual }\end{array}$ & $\begin{array}{l}\text { Standard } \\
\text { Care }\end{array}$ & $\begin{array}{l}\text { Unique } \\
\text { intervention }\end{array}$ \\
\hline \multirow[t]{2}{*}{ Knowledge } & Provide general information & $v$ & $v$ & \\
\hline & Enhance memory \& understanding: graphics & $v$ & & $v$ \\
\hline \multirow[t]{4}{*}{ Awareness } & Risk communication & & $v$ & \\
\hline & Feedback of MEMS-reports & $v$ & & $v$ \\
\hline & Direct feedback of behavior & & $v$ & \\
\hline & Clinical feedback & $\checkmark$ & $v$ & \\
\hline Social Influence & Activate norm of important others & $v$ & & $\mathrm{v}$ \\
\hline \multirow[t]{3}{*}{ Attitude } & Persuasive argumentation & $v$ & $v$ & \\
\hline & Reinforce behavioral progress & $v$ & & $v$ \\
\hline & Reinforce motivational progress & $v$ & & $v$ \\
\hline \multirow[t]{2}{*}{ Self-efficacy } & Practice skills & $v$ & & $v$ \\
\hline & Plan coping responses & $v$ & $v$ & \\
\hline \multirow[t]{2}{*}{ Intention } & Tailored medication schedule & & $v$ & \\
\hline & Organize social support & $v$ & & $v$ \\
\hline Action control & Use of cues to remind intake & $v$ & $v$ & \\
\hline \multirow[t]{2}{*}{ Maintenance } & Goals for maintenance of behavior & $v$ & & $v$ \\
\hline & Relapse prevention & $v$ & & $v$ \\
\hline \multirow[t]{3}{*}{ Facilitation } & Provide functional materials & $v$ & & v \\
\hline & Continuous professional support & & $v$ & \\
\hline & Cope with side-effects & $v$ & $v$ & \\
\hline
\end{tabular}

Note: The first column displays the determinants targeted by the coded techniques in column 2 . The third column shows the techniques coded in the intervention manual and the fourth column shows the techniques applied during standard care. The fifth column displays the unique intervention techniques that differentiated the intervention from standard care. The total range of techniques to which the intervention group was exposed (i.e., total intervention quality) is the sum of the standard care and unique techniques. 


\section{CHAPTER 6}

Figure 2a. Proportions of patients with an undetectable viral load in the control $(0)$ and intervention $(\bullet)$ groups as a function of quality.

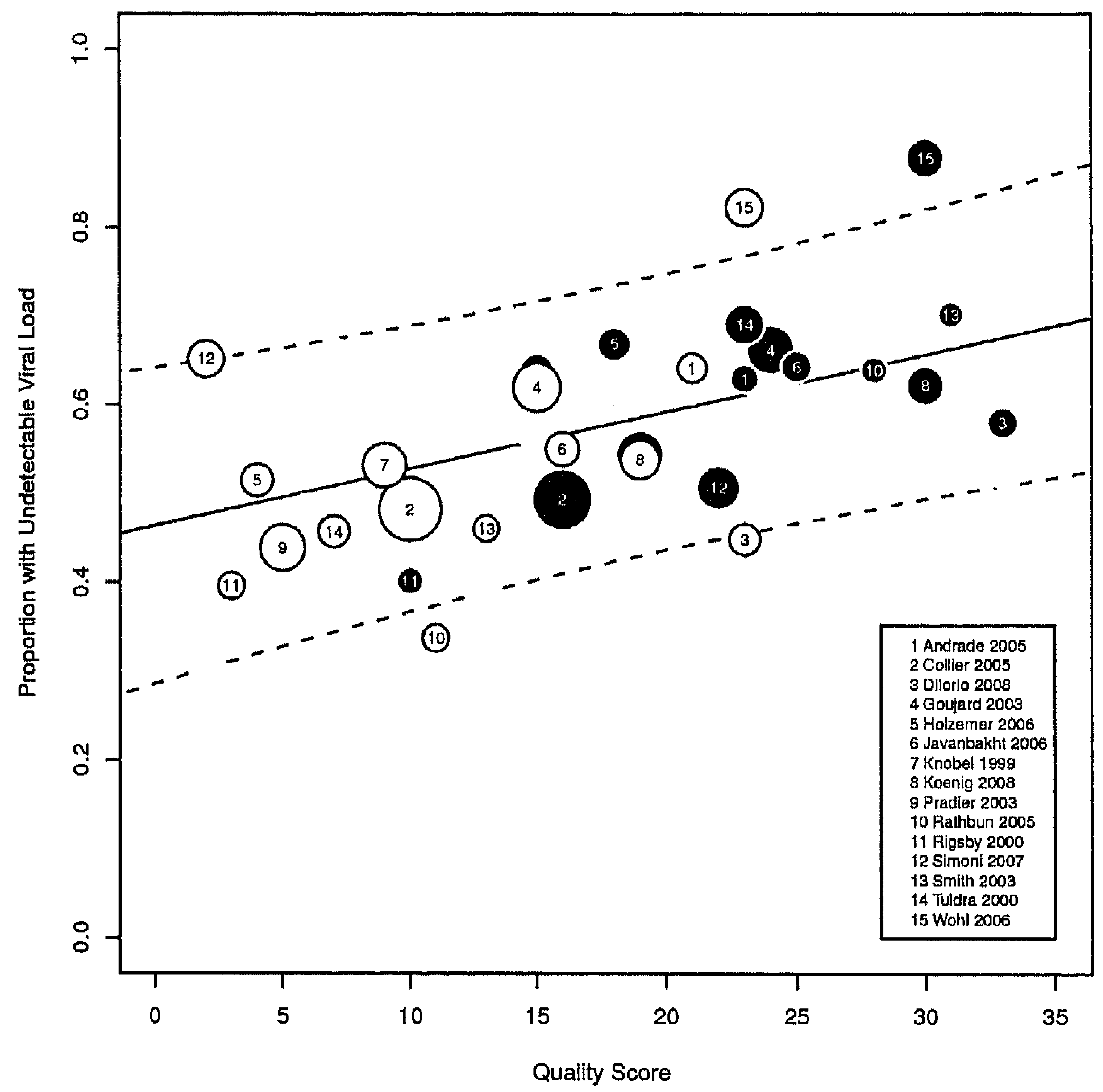

Figure legend: The solid line indicates the predicted average success rate (dashed lines $95 \% \mathrm{Cl}$ ). The point sizes are given relative to the sample sizes and points have been adjusted for the other moderators in the analysis (observed success rates shown in gray). 
How adherence care impacts HIV-treatment outcomes

Figure $2 \mathrm{~b}$. Proportions of patients with $\geq 95 \%$ adherence in the control $(0)$ and intervention ( $\bullet$ ) groups as a function of quality.

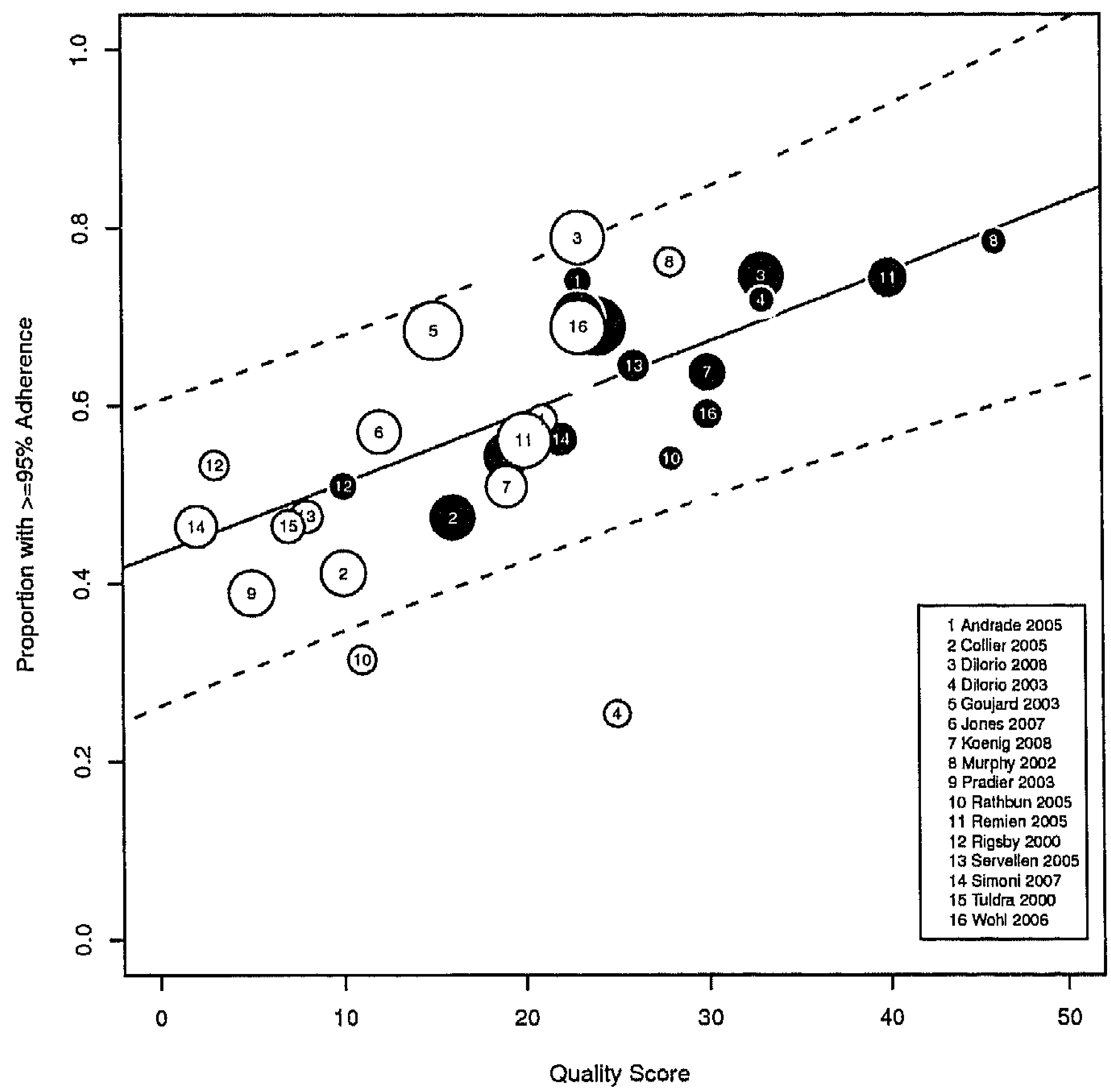

Figure legend: The solid line indicates the predicted average success rate (dashed lines $95 \% \mathrm{Cl}$ ). The point sizes are given relative to the sample sizes and points have been adjusted for the other moderators in the analysis (observed success rates shown in gray). 


\section{CHAPTER 6}

Table 2. Meta-regression results for the success rate analyses

\begin{tabular}{|c|c|c|c|c|c|c|}
\hline & \multicolumn{3}{|c|}{ Viral Load } & \multicolumn{3}{|c|}{ Adherence } \\
\hline & Estimate & $95 \% \mathrm{Cl}$ & $p$-value & Estimate & $95 \% \mathrm{Cl}$ & p-value \\
\hline Quality & .006 & .002 to .011 & $<.01$ & .008 & .005 to .011 & $<.001$ \\
\hline Dominant Ethnicity & -.274 & -.435 to -.113 & $<.001$ & -.048 & -.181 to .084 & .48 \\
\hline $\begin{array}{l}\text { Treatment } \\
\text { Experience }\end{array}$ & -.067 & -.199 to .065 & .32 & -.168 & -.281 to -.055 & $<.01$ \\
\hline Baseline Selection & -.124 & -.430 to .181 & .42 & -.163 & -.310 to -.016 & .03 \\
\hline Mean Study Year & -.001 & -.045 to .042 & .95 & .029 & -.005 to .063 & .09 \\
\hline Percent Dropout & .001 & -.003 to .005 & .66 & -.004 & -.007 to -.000 & .03 \\
\hline $\begin{array}{l}\text { Viral Load Detection } \\
\text { Limit }\end{array}$ & .163 & .008 to .317 & .04 & & & \\
\hline $\begin{array}{l}\text { Adherence } \\
\text { Instrument }\end{array}$ & & & & -.478 & -.590 to -.366 & $<.001$ \\
\hline
\end{tabular}

Notes: dominant ethnicity coded 0 for Caucasian and 1 for Non-Caucasian samples; treatment experience coded as 0 for patients starting and 1 for patients continuing treatment; baseline selection coded 0 for no selection and 1 for selection of patients with treatment problems at baseline; viral load detection limit coded as 0 for less than 400 and 1 for 400 copies/mL; adherence instrument coded as 0 for self-reports and 1 for MEMS-caps.

\section{Predicting the rate differences}

We expected that the difference in quality between intervention and standard care (i.e., the unique intervention quality) would predict the difference in viral load or adherence success rates between the control and intervention groups (i.e., the rate differences). Moreover, we predicted that this unique intervention quality would be a more accurate predictor of the rate differences than the unadjusted intervention quality score (i.e., manual quality).

When we regressed the viral load rate differences on unique intervention quality, differential dropout, and relative contact intensity, one strong outlier emerged (studentized residual of $z=-3.57$ ): an intervention with a high quality score but a negative effect on viral load. This was the only intervention delivered by trained peers instead of health care professionals, which may have had a "boomerang effect" (Fisher et al., 2002). After excluding this study, unique intervention quality was a strong predictor of the rate differences (slope coefficient $=.015$, $95 \% \mathrm{Cl}: .003$ to $.027, p=.02)$, while the other moderators were not $(p=.83$ and $p=$ .88 , respectively). Although manual quality was also a significant predictor $(p=.03)$, 
unique intervention quality had superior predictive power compared to manual quality $\left(R^{2}=.78\right.$ versus $\left.R^{2}=.58\right)$.

When we regressed the adherence rate difference on the same predictors, again one outlier was present (studentized residual of $z=2.35$ ): a medium quality study with an extremely high effect size. The outlier came from a small study $(\mathrm{N}=$ 17) and was excluded from the analysis. Although the predictive power of the model was lower than for viral load $\left(R^{2}=.29\right)$, again unique intervention quality was predictive of the rate differences (slope coefficient $=.015,95 \% \mathrm{Cl}: .005$ to $.025, \mathrm{p}<$ $.01)$, while contact intensity was not $(p=.94)$. Differential dropout also helped to account for some of the heterogeneity (slope coefficient $=-.01,95 \% \mathrm{Cl}: .02$ to -.00 , $p=.04)$. On the other hand, manual quality was not significant $(p=.12)$ and again had lower predictive power $\left(R^{2}=.17\right)$.

Figures $3 a$ and $3 b$ show the rate differences as a function of the unique intervention quality for both outcome measures. The two outliers (one in each analysis) that do not appear to fit the models are clearly identifiable in these plots. Excluding both outliers from the success rate analyses reported in the previous section did not change the pattern of results, except that unique intervention quality explained considerably more heterogeneity in the viral loads (estimate .010 , $p<.001$ instead of $.006, p<.01$ ). 


\section{CHAPTER 6}

Figure 3a. Difference in the proportion of patients with an undetectable viral load in the control versus the intervention group as a function of unique intervention quality.

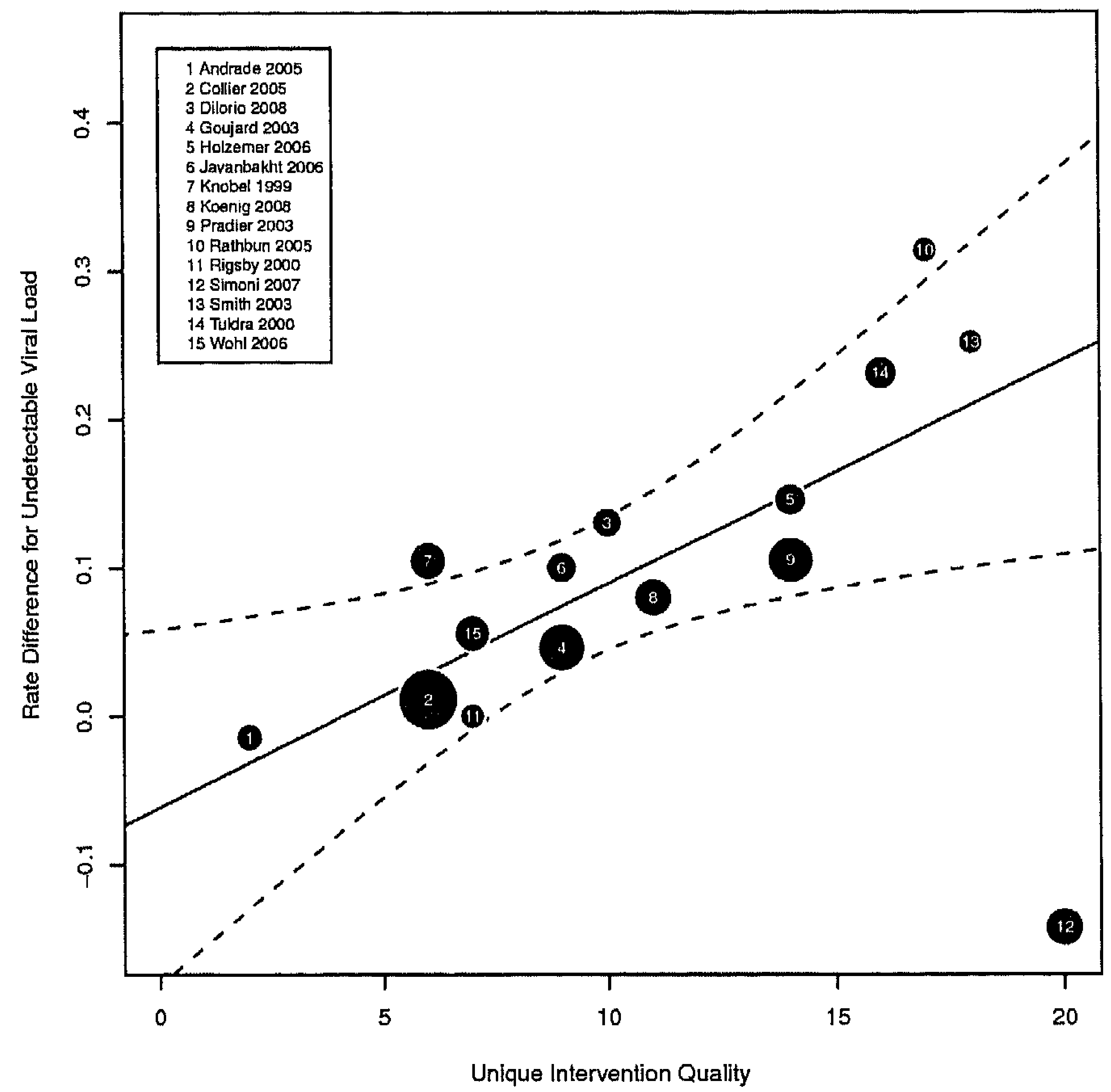

Figure legend: The solid line indicates the predicted average rate difference (with $95 \%$ confidence interval given by the dashed lines). The point sizes are given relative to the sample sizes. 
How adherence care impacts HIV-treatment outcomes

Figure 3b. Difference in the proportion of patients with $295 \%$ adherence in the control versus the intervention group as a function of unique intervention quality.

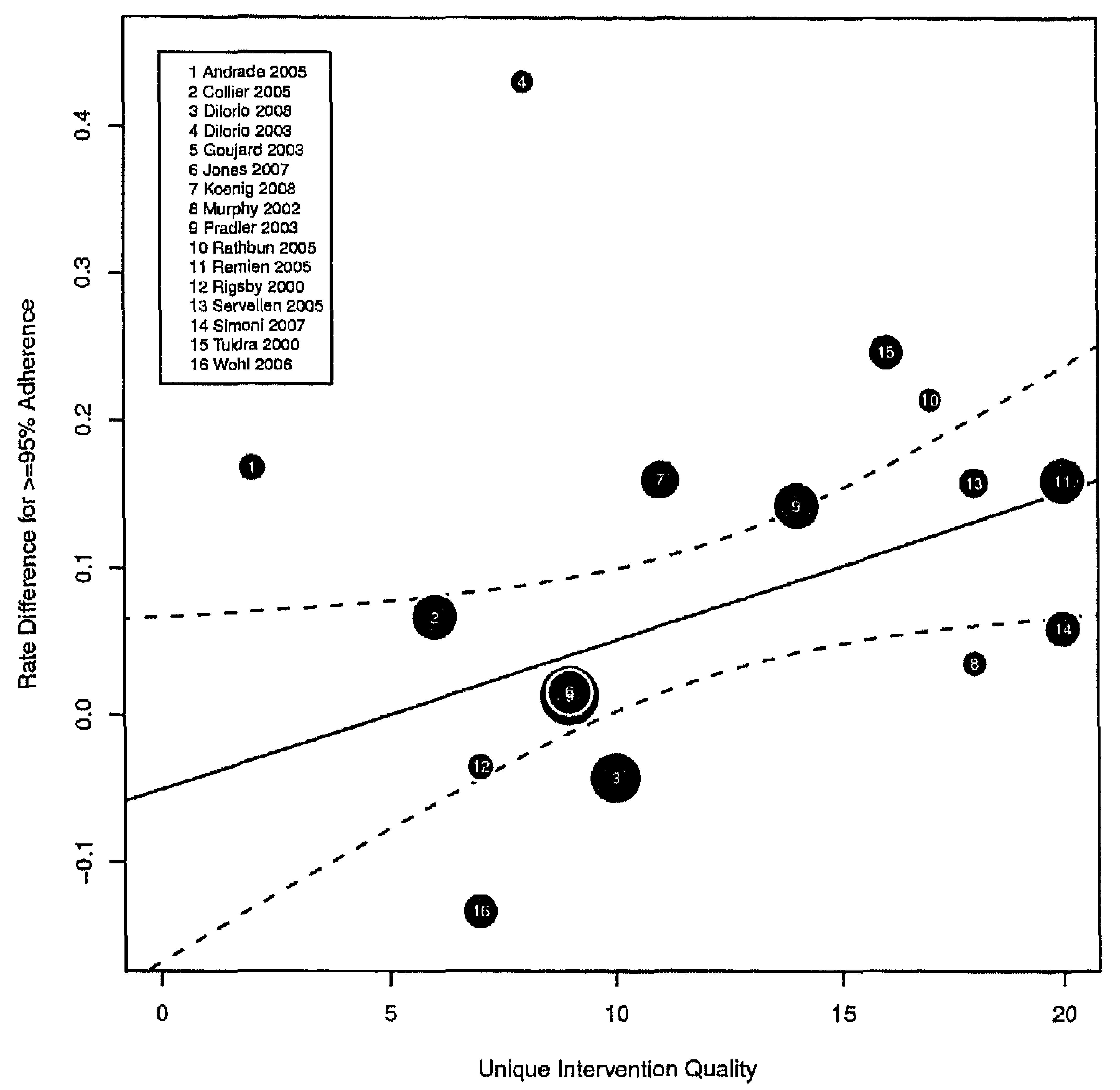

Figure legend: The solid line indicates the predicted average rate difference (with $95 \%$ confidence interval given by the dashed lines). The point sizes are given relative to the sample sizes. 


\section{CHAPTERG}

\section{The impact of variability in SCQ on treatment and intervention effects}

The results from the analyses above indicate that the rate (difference) for viral load increases on average by approximately $1 \%$ to $1.5 \%$ points for each additional point in (unique) quality. Implementing the strongest available standard care in an HIVclinic currently delivering the weakest level of care could, therefore, result in 20$30 \%$ points increase in the viral load success rate (range in SCQ 3-23). Examining the total quality range (i.e., including the interventions), the impact of adherence care is estimated at $30-45 \%$ points (complete range in quality $3-33$ ). Note that whereas the adherence analyses show larger quality ranges to be effective (standard care: 2-28; including interventions: $2-46$ ), the quality ranges in the viral load meta-analyses are restricted due to missing data.

The considerable variability in care provided to the control groups also impacted the intervention effects. The overlap between experimental and standard care ranged from $0-50 \%(0-53 \%$ in terms of quality points). This implies that the effect sizes reported by some interventions could have been up to twice as large if they had been tested in the study setting with the weakest observed standard care.

To examine the expected effectiveness of the interventions under equal SCQ conditions, we identified the typical BCTs applied in low, medium, and high standard care and the unique quality of each intervention under these conditions. Using $1.25 \%$ points as the estimated increase in the rate difference for each point increase in unique intervention quality (as a compromise between $1 \%$ and $1.5 \%$ ), we computed three estimated rate differences for each study included in the final analyses. As Figure 4 shows, most interventions are expected to improve clinical outcomes in settings with low levels of standard care. However, with increasing SCQ the effectiveness of interventions reduces considerably. In settings with the strongest standard care, just a few labor-intensive interventions are expected to yield improvements greater than $10 \%$ points in viral suppression rates.

\section{DISCUSSION}

This meta-analysis showed that both the intervention and control groups in HAART adherence interventions trials were exposed to effective care that impacted medication adherence and clinical outcomes. Whereas most interventions included in the study are likely to increase success rates in clinics with weaker standard care, these effects decrease considerably with increasing standard care quality. With the highest observed standard care level being twice that of the mean SCQ observed ( 28 
Figure 4. Expected viral load rate differences compared to low, medium, or high quality standard care. The estimated effects under the observed SCQ conditions are indicated by the circles $(0)$.

Expected Rate Differences under Difference Levels of Standard Care

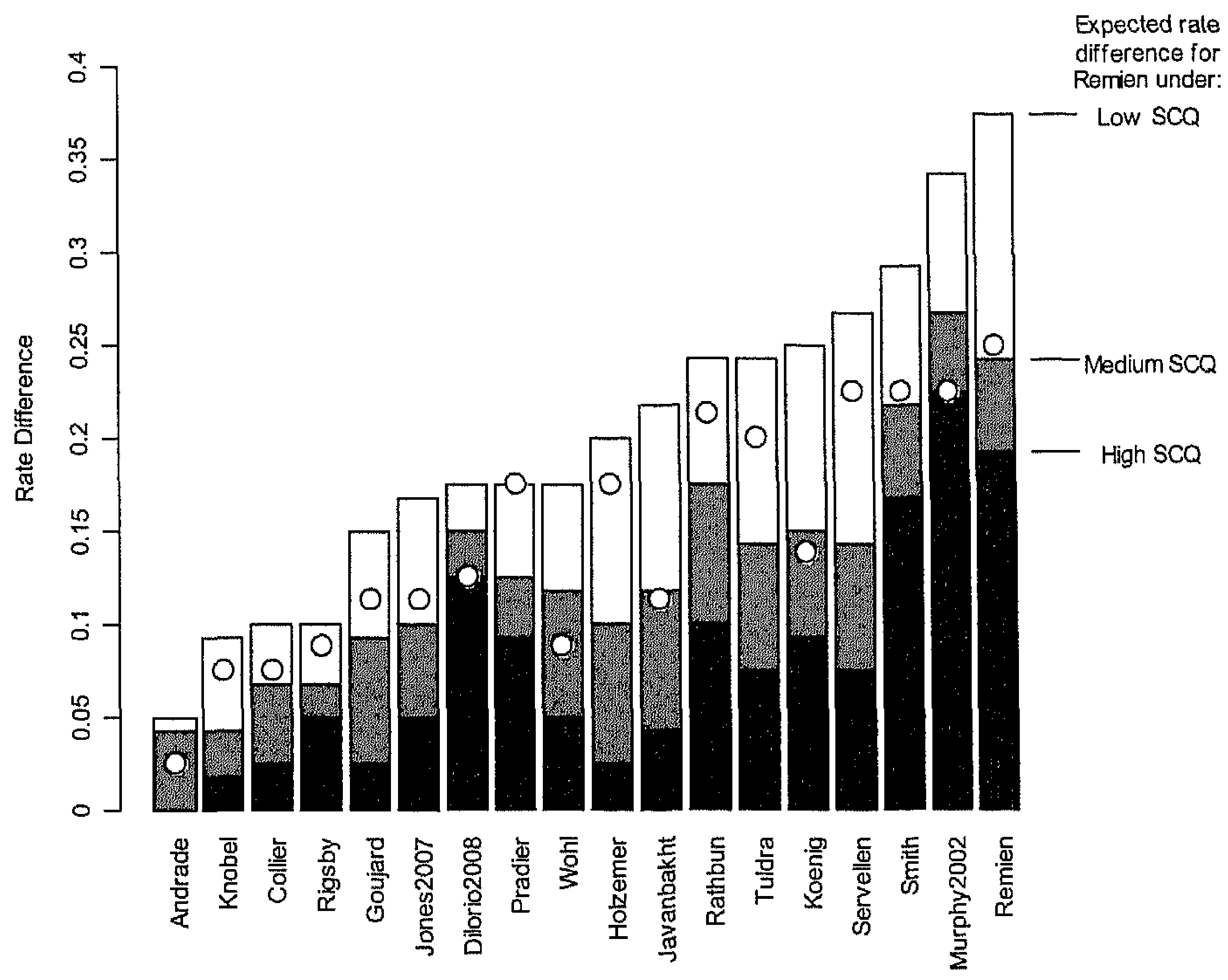

Note: Low SCQ: Instructions for taking medication, feedback clinical results, encouraging to adhere; Medium SCQ adds: Verbal and written information (HIV, HAART, adherence, dealing with side effects) and tailored medication planning including cues, week boxes, social support; High SCQ adds: Continued attention for adherence problems and solutions during follow-up, provision of materials (e.g., alarm devices) and a phone number to contact a health care professional in case of problems. Assumption: all interventions are delivered $\geq 12$ weeks and on top of standard care.

points versus 14), the wide-spread adoption of current best practice would be expected to increase HAART treatment effectiveness in developed countries by 14$21 \%$ viral load points; an improvement that justifies considerable investment in additional personnel, training, and equipment (Goldie et al., 2003; Scalera et al., 2002). Since only a few existing interventions are then expected to further improve treatment outcomes, standardization of routine care by adoption of alreadyimplemented best practice seems at present the most cost-effective and pragmatic 
approach to improving adherence and viral load outcomes (for best practice details, see de Bruin et al., in press).

In addition to these clinical implications, the results have two more general implications. First, systematic analysis of the content of care provided to active control groups is prerequisite to understanding the added value of behavior change interventions. When designing new behavior change interventions researchers should therefore first assess and then address deficits in existing standard care by selection of carefully chosen, theory- and evidence-based BCTs (Abraham \& Michie, 2008; Bartholomew et al., 2006; Green \& Kreuter, 1999). Second, since variability in care provided to active controls is also present in other domains (Ismail et al., 2004; Wagner \& Kanouse, 2003), comparing intervention effects in meta-analyses of behavior change interventions seems only meaningful after controlling for variability in standard care provided to control groups.

The analyses yielded other noteworthy results for the domain of HAART adherence. The success rate analyses showed that non-Caucasian patients had a considerably lower chance of achieving clinical success. Unexpectedly, this was not found for adherence. We can think of two possible explanations for these findings. First, something other than non-adherence could cause the treatment to be less effective among non-Caucasian patients, such as differences in patients' clinical status at the start of the treatment (Nellen et al., 2004). Second, the adherence measurement instruments may have lacked cross-cultural validity, so that differences in adherence between Caucasian and non-Caucasian samples were not detected. Our findings clarify that this difference is not due to poorer standard care or other confounders examined here.

Mean study year approached significance for adherence, but not for viral load. We used this variable as an indicator for the increasing effectiveness and reduced complexity of HAART regimes during the last decade. This null finding suggests that the range of mean study year (i.e., 1996-2004) may have been too restricted to detect an effect of time for viral load, or something other than medical improvements - such as advances in standard adherence care - has been a leading cause of the increasing effectiveness of HIV treatments observed in large HIV-cohorts (Antiretroviral cohort Collaboration, 2008; Gras et al., 2008)

It is also noteworthy that a $48 \%$ higher adherence success rate was observed for self-reports compared to MEMS-caps. It is well-known that self-reports may lack sensitivity and MEMS-caps may lack specificity (Liu et al., 2001; Melbourne et al., 1999; Paterson et al., 2002). However, the size of this difference is worrying and calls for improved measurement strategies.

We also found that the moderator "intensity" was not significant in the rate difference analyses. This null finding suggests that the observed effects are not 
attributable to the amount of time patients spend with the health care professional, which is an assumption in study designs with attention-control groups, but to how health care professional utilize that time (i.e., by applying effective BCTs directed at important determinants of the health behavior).

Our study has certain limitations. Foremost among these is the modest number of RCTs included in the final analysis. Whereas 34 eligible RCTs were retrieved and 33 authors readily responded to our request for additional study information, only 20 studies could be included in the final analyses. While a few studies did not meet a priori defined criteria and some outcome data could not be obtained, three intervention descriptions were too vague to allow coding and standard care descriptions were unavailable for 11 studies. This data loss highlights an urgent need to improve intervention and standard care descriptions available for published trials, an issue that has recently received considerable attention (Abraham \& Michie, 2008; Boutron et al., 2008; Davidson et al., 2003; de Bruin et al., in press; Schaalma \& Kok, 2009) Such descriptions should allow readers to accurately identify which $\mathrm{BCT}$ s were applied in each condition and with what purpose. Only then can readers determine against which level of standard care any particular intervention was tested, and so estimate the possible effectiveness of an intervention in other settings (Wagner \& Kanouse, 2003) Moreover, meta-analysts can then more easily control for variability in standard care and, thereby, accurately assess the added value of behavior change interventions.

In conclusion, the present meta-analysis showed how adherence interventions impact behavior and clinical outcomes. Moreover, the results showed that the care provided to active control groups impacts effect sizes reported by intervention trials, which suggests that future meta-analyses should control for variability in care provided to active control groups. Finally, the findings suggest that substantial increases in treatment effectiveness could follow from the widespread adoption of current best standard care practice. 
CHAPTER 7

General Discussion 


\section{GENERAL DISCUSSION}

Since 1996, HIV can be effectively suppressed with Highly Active Antiretroviral Therapy. However, the effectiveness of HAART depends largely on how accurately patients take the medication. With $90.95 \%$ adherence being considered sufficient for restoration of the immune system and a minimal risk on development of resistant viral strains, research shows that approximately $50 \%$ of the patients is insufficiently adherent. Behavioral interventions that effectively support patients in improving adherence can therefore have a large impact on the life expectancy and quality of life of HIV-positive patients, and on the cost-effectiveness of HIV-care. However, despite numerous studies on HAART adherence predictors and adherence supporting interventions, scientific evidence for effective adherence care is rather poor. Hence, and all the more urgent considering the steadily growing number of people receiving HAART, this field could benefit considerably from evidence to guide the development of effective and feasible adherence care to improve patients' health and quality of life.

The main objective of the research described in this thesis was to expand the evidence-base for adherence care through the theory- and evidence-driven development and evaluation of adherence supporting interventions. Moreover, some studies in this thesis were designed to also contribute to theoretical and methodological issues relevant to the larger domain of behavioral science.

\section{The main findings from the clinical studies}

As laid out in the introduction of this thesis, one line of research concerned the development and evaluation of a new, theory-and evidence-based intervention to enhance adherence to HAART. This Adherence Improving self-Management Strategy (AIMS) was developed using literature reviews in the domain of (HAART) adherence and behavioral theories. Moreover, expert input was provided by HIV-nurses to fine-tune the intervention to known patient needs and problems, and to the time and competences available during routine clinical care. The intervention was then pilot-tested and the results showed positive effects of the intervention on adherence, measured electronically with MEMS-caps. Moreover, patients and HIVnurses evaluated the intervention positively and the intervention seemed to fit with the time and competences available to HIV-nurses during routine clinical care. Finally, direct observation of the intervention contacts between the patients and the HIV-nurses suggested that no important adherence difficulties were overlooked by the AlMS-intervention. Building on these encouraging findings, we decided to 
study the effectiveness of AIMS more rigorously in a randomized controlled trial (RCT).

During the 9-month RCT, the HIV-nurses delivered the intervention during routine clinical visits without the presence of a researcher. The study clinic provided high quality standard care, which explains the relatively high levels of adherence and viral suppression in the study sample at baseline. Notwithstanding the quality of standard care provided to the control group during the trial, adherence and viral suppression rates improved significantly during the 3-month intervention period, and the adherence improvements remained stable during follow-up. Moreover, the effects of treatment assignment on viral load were mediated by the improvements in adherence, which confirmed the assumption of a causal relationship between intervention assignment and improved adherence, and between improved adherence and the treatment effect on viral load. Hence, this study provided strong support for the efficacy and clinical relevance of AIMS.

Besides efficacy, an important objective of the RCT was to evaluate whether the HIV-nurses could deliver the intervention during the time available for routine clinical visits. The data showed that the mean duration of the first intervention visit was approximately twice as long as a usual care visit, whereas the duration of consecutive intervention contacts was similar in duration. Hence, although we consider the additional time investment reasonable when taking the intervention effects into account, the objective to deliver all the intervention visits within the time available during routine clinical care was not fully accomplished.

The longer duration of the first visit can be attributed to several factors. First, the HIV-nurses reported going through a learning curve during the study. Therefore, with increased experience the duration of the intervention visits may decrease. Second, due to the examination of the MEMS-reports in the intervention group, a larger number of non-adherent events were detected compared to the control group. Just as in usual care, this is reason for additional intervention activities that cost time. Third, independent of the learning curve and the detection of nonadherence, the intervention manual may contain more (time consuming) activities than usual care. Following from these explanations we expect that, although increasing experience with AIMS can reduce the duration of the intervention contacts to a certain extent, it may be necessary to allocate some additional time for the first intervention visit compared to a usual care visit.

Finally, we also evaluated whether the HIV-nurses delivered the intervention adequately. Although the data available for this evaluation were inferior compared to the pilot study, namely structured intervention dossiers and nurse interviews during the RCT versus direct observation and nurse interviews during the pilot, they suggest that the main intervention components had been adequately delivered. 
However, in line with the HIV-nurses' reports of a learning effect during the trial, the process data do also show that there is some room for improvement. Hence, booster and advanced courses in AIMS counseling may be useful for further improving nurses' knowledge and skills to deliver the intervention effectively and within the required time.

During the randomized controlled trial, patients also completed questionnaires that contained cognitive determinants of adherence, and preparatory (i.e., carrying medication along when not at home) and self-regulatory activities (i.e., selfmonitoring of accuracy of medication intake and devising solutions when own nonadherence was detected). These data were primarily used to study a theoretical idea among a clinical sample. The main objective of this analysis was to test whether preparatory and self-regulatory activities explain how patients translate their behavioral intentions into actual behavior, and thereby bridge the so-called gap between people's intentions and future behavior that is often found in studies applying intention-behavior models.

We examined the data collected at baseline and post-intervention in the control group and found that both "carrying medication along" and "selfmonitoring" simultaneously mediated the intention-behavior relation. Moreover, the combined activities explained an additional $25 \%$ of variance in behavior on top of intention and other common determinants included in the analyses. These findings suggest that self-regulatory activities and preparatory behaviors mediate the intention-behavior relationship, and that these activities explain a unique portion of variance in behavior not accounted for by intention and the other predictors. Hence, future determinant studies could more successfully predict behavior if they include relevant preparatory and self-regulatory activities in their analyses. Moreover, these findings show that self-monitoring of adherence and carrying medication along are functional activities for high HAART adherence, and that even under high quality standard care conditions these behaviors are not optimally executed. Therefore, adherence interventions may benefit from including these activities as behavioral objectives in the program.

The outcomes of the intention-behavior study warrant reflection on whether AIMS does effectively promote self-monitoring and stimulates patients to carry their medication along. Part of the AIM-strategy is to ask patients to self-monitor their medication intake in the upcoming period in order to allow a more detailed discussion of adherence barriers at the next visit. In addition, adherence is monitored with the MEMS-caps after which problems are retraced during the intervention visits, which should partially fulfill the role of active self-monitoring. The preparatory behavior carrying medication along is not systematically targeted 
by AIMS, but addressed only if patients report that non-adherence events are caused by not having medication available when outdoors. The patients and HIVnurse then discuss possible solutions for this problem (e.g., store spare medication doses in one's car, purse, or at work). Since the questionnaire study shows that these activities contribute substantially to HAART adherence, additional analyses over the complete study sample could be conducted to examine whether treatment condition moderates the effects of self-monitoring and carrying medication at baseline on adherence at post-intervention. If this is not the case, the effectiveness of AIMS could be improved by more extensive intervention activities targeting important determinants of self-monitoring and of carrying medication along when patients are outdoors.

\section{Strengths and limitations of the clinical studies}

The clinical studies have several strengths and limitations. Strengths of AIMS are that it has been developed to fit with HIV-nurses competences, and that it seems to require only a limited time- investment on top of routine clinical care. In addition, AIMS is theory- and evidence-driven, and all techniques are tailored to patients' individual problems and needs. Moreover, the intervention was pilot-tested before it was studied more rigorously in a randomized clinical trial in which the effects on clinical and behavioral outcomes were assessed objectively. Also due to the modest dropout rates, the RCT was sufficiently powered to show significant intervention effects on adherence and on viral load. It is important to note that these effects were obtained in a setting with high-quality standard care, a challenging setting to demonstrate advantages of adherence interventions. Finally, both the HIV-nurses and the patients indicated that the AIMS-intervention and the MEMS-reports were useful and valuable. Hence, AIMS has proven effective, seems to fit in standard care, and to be appreciated by the end-users. There was one characteristic of AIMS, however, that received both positive and negative feedback from the patients: The MEMS-cap.

The MEMS-cap has a number of strengths compared to other adherence measurement tools such as self-reports, pill counts and the measurement of therapeutic drug levels. The MEMS measures adherence objectively and it provides an unparalleled, detailed insight in patient behavior over long time periods. These data permit rigorous scientific evaluations of adherence, but also allow betterinformed medical decisions and adherence interventions in clinical practice. Hence, the MEMS-data were considered very valuable by the researchers, the health care professionals, and the patients. Unfortunately, the MEMS-cap plus pill-bottle is 


\section{CHAPTER 7}

rather bulky and resembles a medication bottle, which conflicts with some HIVpositive patients' preferences for discrete medication storage, or medication containers with multiple compartments. Even despite several strategies to facilitate MEMS-use in the RCT, its design was the primary reason for $20 \%$ of the patients to reject study participation. Moreover, it was a barrier to consistent accurate use among some patients, and half of the patients who dropped out the study reported to do so because of the MEMS-cap (note, however, that the total dropout was only $13 \%)$. Hence, the quality of the data and possibly the type of patients included in the study was limited by the design of the MEMS-cap.

The representativeness of our study sample for the general population studies was also affected by our inclusion criterion of Dutch-speaking, Caucasian patients only. Approximately $40 \%$ percent of the Dutch HIV-positive population originates from Surinam, the Antilles, and Africa, and research shows that treatment problems are more frequent among these "non-Caucasian" patient groups (Nellen et al., 2004). Although a randomized pilot-study among non-Caucasian was planned directly following the RCT, the RCT progressed slower than anticipated. We therefore decided to postpone the study of AIMS among non-Caucasian patients to a future trial.

The above mentioned limitations also apply to the questionnaire study. Moreover, the sample size in this study was modest, which reduced statistical power to detected relevant predictors. Nevertheless, we found that two out of three activities mediated the intention-behavior relation, both in regression analyses and in non-parametric bootstrapping analyses, which can be applied to smaller samples with more confidence. Moreover, the analyses were carefully controlled for potential confounders, and the behavioral measure was objective and computed over a long time period (i.e., one month). Hence, although the implications of these findings should be framed tentatively, this study shows a potentially promising avenue of research for improving the predictive value of behavioral theories, and for the design of more effective HAART adherence interventions. 


\section{Main conclusions from the clinical studies}

Despite high levels of adherence care in the study clinic, a considerable proportion of patients display suboptimal adherence. The AIMS intervention is a theory-and evidence-based intervention which has proven effective and feasible compared to high-quality standard care. Moreover, AIMS largely fits in routine clinical care and it has been evaluated positively by the end-users. The effectiveness of AIMS could be further improved by additional nurse-training and possibly through increased attention for preparatory and self-regulatory activities.

AIMS has not yet been tested among the Surinam, Antillean and African patients in the Netherlands, among whom treatment success rates are lower and socio-demographic circumstances seem more demanding (e.g., fear for social exclusion following disclosure, unemployment, legal issues). Hence, future research should focus on adapting AIMS to the relevant differences between patient groups, and on testing its effectiveness and acceptability also among the non-Caucasian patients facing the challenge of achieving and maintaining the high levels of adherence required for long-term effectiveness of HAART. Moreover, although designed to fit in routine clinical care, AIMS has yet to prove its costreffectiveness before wide-spread adoption and implementation should be recommended. We would like to note that the adoption of AIMS should include creating a supportive management and ICT-environment for, and a specialized training of the HIV-nurses. Moreover, HIV-nurses will go through a learning curve when delivering AIMS during which support and booster trainings may be useful. Hence, the adoption and implementation of AIMS should be well-coordinated and planned. Finally, future research and the implementation of AIMS in routine clinical care would be greatly facilitated by technological innovations to develop more patient-friendly electronic monitoring devices to measure adherence.

\section{Main results from the meta-analyses}

The second line of research described in this thesis consists of meta-analytic studies examining the content and effectiveness of care provided to patients in HAART adherence intervention trials. As already reported in the introduction and the previous chapter, previous reviews and meta-analyses in this field have been quite unsuccessful in explaining the observed differences in intervention effects between studies, leaving researchers and health care professionals with little evidence to improve their practice. An explanation for this lack of findings could be that metaanalyses of interventions usually study the content of interventions in detail, while 
ignoring the standard care provided to the control groups in the reviewed studies. However, if the quality of standard care varies between studies and impacts treatment outcomes, intervention effects cannot be accurately interpreted, compared, nor generalized to other settings without controlling for the care provided to the control groups.

The objective of the first meta-analysis was to study whether the quality of standard care provided to control groups in HAART adherence trials could be reliably assessed, varied between studies and was related to the proportion of patients with an undetectable viral load (i.e., the success rate). Standard care quality was determined based on the coding of its active content (i.e., the theoryand evidence-based behavior change techniques (BCT's) targeting important determinants of adherence), which was then weighted for personal relevance and predominance. The results showed that the standard care quality instrument was a reliable tool, and that the quality of standard care varied considerably between the reviewed studies. In addition, standard care quality was significantly related to clinically relevant variations in treatment success rates, meaning that patients chances on an undetectable viral load depended to a large extent on the quality of standard care provided to them. These findings suggest that meta-analysts synthesizing intervention studies cannot assume that all interventions are being tested under equal conditions, but that it is necessary to assess and control for variability in standard care and thereby obtain more accurate estimates of intervention effects. Moreover, this is the first meta-analysis in the HAART adherence field to show a relation between the content of adherence care and treatment outcomes.

The objective of the second study was to examine whether the quality of HAART adherence interventions tested in randomized controlled trials predicted treatment success rates, after controlling for variability in standard care provided to control groups. Both standard care quality and intervention quality were reliably coded, and the respective quality scores were again based on weighing the behavior change techniques for personal relevance and predominance. The first step in the analyses was to examine whether both standard care quality as well as total intervention quality (i.e., the sum score of all techniques delivered to intervention patients, namely those provided in standard care and in the intervention) predicted the proportion of patients with adherence $\geq 95 \%$, and the proportion of patients with an undetectable viral load (i.e., the success rates). The next step was to examine whether the difference between standard care quality and intervention quality predicted the difference in success rates between both conditions (i.e., the rate differences, which was used as the effects size). Finally, the results were used to model the expected intervention effects of each intervention included in the 
meta-analyses under similar standard care quality conditions (i.e. low, medium and high quality standard care).

The first analyses showed that standard care quality and intervention quality scores reflected the same underlying quality dimension, which predicted a considerable amount of heterogeneity in the adherence and viral load success rates. Interestingly, half of the control groups were exposed to adherence care that was equal or superior to the quality and effectiveness of the adherence care provided to half of the intervention groups. These success rate analyses demonstrated that both standard care and intervention care impacted patient behavior and treatment outcomes, and that testing the effectiveness of a behavior change intervention in a clinical trial is actually a test of the hypothesized superior quality of one intervention ("intervention care") over another ("standard care").

The success rate analyses also yielded several noteworthy outcomes regarding methodological and population differences in treatment success rates. Most notably were the considerably lower levels of viral suppression among non-Caucasian patients, which did not seem to be caused by lower levels of adherence. Hence, it is imperative that future research uncovers the exact causes of these health disparities. Another striking finding was the large difference in adherence success rates assessed by MEMS-caps compared to self-reports. This result calls for additional measurement procedures to improve the sensitivity of adherence selfreports and the specificity of MEMS-monitoring. The strategies discussed in the RCT are an example of such procedures. Finally, it is widely assumed that medical advances, which have reduced the complexity of HAART regimen and increased HAART efficacy, are the primary cause for the advances in clinical success rates and increasing life expectancy observed among HIV-infected patients in rich countries since 1996 (Antiretroviral Cohort Collaboration, 2008) However, we could not find a relation between treatment success rates and the year in which the studies were conducted. We did find a strong relation between the quality of adherence care and clinical outcomes, which suggest that perhaps advances in adherence care rather than medical advances have been the primary cause of the improvements in clinical outcomes observed among HIV-infected patients treated with HAART since 1996.

In the second set of analyses, the difference in quality between intervention and standard care was used to predict the difference in success rates (i.e., the rate difference). These analyses indeed showed that the unique intervention quality was an accurate predictor of the observed effect sizes, which further validated the quality measure. Moreover, the unique intervention quality proved to be a more accurate predictor of the intervention effects than the unadjusted intervention quality scores, which is commonly used in meta-analyses of interventions. Thus, meta-analyses of behavior change interventions, or those examining the 
effectiveness of sets of behavior change techniques or single techniques, are warranted to first assess whether these techniques are indeed unique for intervention care. Otherwise, these meta-analyses may over- or underestimate the effectiveness of (sets of) behavior change techniques, or fail to accurately explain why some intervention are more effective than others.

Finally, we determined the unique intervention quality of each meta-analyzed intervention compared to low, medium and high levels of standard care quality. Using these unique intervention quality scores and the results obtained from both the success rate and rate difference analyses, we computed the expected effect sizes of all meta-analyzed interventions under the same standard care quality conditions (i.e., low, medium and high). The resulting "model" (see Figure 4, chapter 6) allows health care professionals to directly compare the effects of each intervention under similar standard care conditions, and to more accurately estimate the probable effectiveness of each intervention in their own setting. Moreover, the model shows that under high standard care quality conditions, only a few labor-intensive interventions are expected to yield improvements in viral suppression greater than ten percent. With the mean level of standard care observed being only half as effective as the highest quality of standard care observed (i.e., current best practice), the widespread implementation of current best practice should increase viral load success rates in developed countries with approximately $14-21 \%$ points. Assuming that already implemented standard care is feasible, and that the estimated gain in viral suppression legitimizes considerable expenses in personnel, training and facilities, we recommend Western HIV-clinics to adopt and implement current best practice as their standard of care.

Even in settings with high quality standard care, the quality and effectiveness of adherence care can be improved. The AIMS intervention was tested in a high standard care quality setting and still improved adherence and viral suppression rates with approximately $15 \%$. Hence, health care providers already delivering current best practice could examine the literature for intervention that have proven to be (cost-)effective compared to high quality standard care. The AIMS intervention described in this thesis could be one of these interventions although, as noted previously, it has yet to be tested among non-Caucasian patient groups.

Besides that the described meta-analyses update previous literature reviews, identify several relevant confounders of treatment success, and demonstrate how future meta-analyses of behavior change interventions could improve their accuracy, the approach of the present meta-analyses towards explaining the intervention effects from the content of the interventions is different from what meta-analyses of behavior change interventions usually do. Normally, metaanalyses regress (sets of) behavior change techniques on the effects sizes reported 
by intervention trials, and then draw conclusions about which of these techniques are effective based on their correlation with the intervention effects. In the present work, however, we focused on an underlying mechanism rather than on (sets of) behavior change techniques, thereby assuming to already know the active content (i.e., theory- and evidence-based behavior change techniques targeting important determinants of the health behavior) and their relative weights (i.e., personal relevance and predominance). This rationale was based on insights from prevailing behavioral theories and on Intervention Mapping principles, which is a systematic approach towards developing health promotion programs (Bartholomew et al., 2006). Hence, the fact that the standard care and intervention quality scores so accurately predicted the observed effects does not demonstrate the effectiveness of specific (sets of) behavior change techniques, but rather of an underlying mechanism: The effectiveness of behavior change interventions depends on the degree to which important determinants of the health behavior are repeatedly targeted by unique, tailored, theory- and evidence-based behavior change techniques.

Besides that the described mechanism is an application of Intervention Mapping principles, it also adds one element to this framework. As we just described, Intervention Mapping promotes the application of theory- and evidencebased behavior change techniques to important determinants of the target behavior(s). Which determinants are most important, meaning that the determinant predicts the health behavior, is normally established through the use of behavioral theory, and through qualitative and quantitative research. Next, intervention developers select one or multiple behavior change techniques from a range of appropriate techniques to target these determinants. Whereas at present intervention developers select behavior change techniques only based on their potential to alter the important determinants, the obtained results suggests that interventionists should also consider whether techniques are already being delivered to the target group (i.e., as part of "standard care"). In the present studies approximately one-third of the authors could not fill out the standard care checklist), and those who could frequently selected behavior change techniques similar to those already applied (especially in settings with high quality standard care). Hence, it could be useful if the Intervention Mapping approach would also recommend intervention developers to make (and report) a systematic assessment of current standard care, to ensure that the selected behavior change techniques are an actual improvement over the care already provided. 


\section{Strengths and limitations of the meta-analytic studies}

Strengths of the meta-analyses are that almost all authors of the included studies responded to our requests for confirming our coding results, and for providing additional study information if data were missing (e.g., outcome data, dominant ethnicity, time point of the immediate post-intervention assessment, and so forth). Moreover, the authors provided, if available, detailed information about the content of standard care and of the experimental interventions. These materials were coded reliably with a comprehensive taxonomy of behavior change techniques.

The outcomes of the analyses presented in both studies were consistent with the study hypotheses, regardless of the dependent variable (i.e., adherence or viral load) or the type of analysis (i.e., success rate or rate difference analysis). Moreover, all the analyses were controlled for potentially important confounders and moderators, to ensure that the observed relations between quality and adherence or viral load were genuine. The consistency in all these findings strongly support the theoretical framework and main conclusions.

Both meta-analyses have one common limitation, which is the number of studies dropping out of the analyses because of missing data. Most of the missing data concerned the content of care provided to the control groups. The dropout reduced the power in our analysis, which was quite modest in the rate difference analyses. Although the theoretical framework and all the success rate analyses support the conclusions drawn from the final rate difference analyses, replication of this work among a larger sample of studies and in other fields would certainly strengthen our conclusions and recommendations.

\section{Main conclusions from the meta-analyses}

The meta-analyses sought to develop reliable tools to assess the quality of standard care and of the experimental interventions targeting HAART adherence, to examine whether adherence and viral load success rates could be explained by the quality of care, and to investigate whether meta-analyses of behavior change interventions need to control for variability in standard care quality in order to more accurately determine the effectiveness of behavior change interventions.

The studies showed that both standard care quality and intervention quality could be reliably assessed. Moreover, the quality of standard and intervention care varied considerably between the studies and impacted the adherence and viral load success rates. Finally, the effect sizes were best understood after controlling for 
variability in standard care quality provided to the control groups in the respective trials. Hence, these studies showed how adherence care impacts treatment outcomes, and that future meta-analyses of (behavior change) interventions should assess and control for variability in standard care quality in order to more accurately estimate the effectiveness of interventions. In addition, the outcomes showed that the wide-spread implementation of current best practice, of which the detailed content is described in the first meta-analysis, could substantially increase treatment success rates in developed countries. Finally, the meta-analyses reveal several potentially important topics for future adherence research, like determining what causes the lower effectiveness of HAART among non-Caucasian patients groups, and how the quality of adherence assessments can be increased.

\section{Overall conclusions and implications}

The number of patients on HAART is large and expanding rapidly. With approximately $50 \%$ of the patients using HAART insufficiently, and only little evidence to guide the development of evidence-based adherence care, effective and feasible adherence interventions are needed. The main objective of the research presented in this thesis was to systematically apply behavioral theory and evidence in clinical trials and meta-analyses to expand the evidence-base for adherence care. The findings in the clinical and meta-analytic studies permit the development of evidence-based guidelines for highly effective adherence care in clinical practice. The wide-spread adoption and implementation of current best practice in developed countries is expected to have a large impact on patients' adherence and viral suppression rates. Moreover, the quality and effectiveness of current best practice could be further enhanced though the application of the AIMS. Hence, also considering the feasibility of these practices, we hope these findings will not only remain a scientific contribution, but that they will also be applied in clinical practice for the betterment of patients' health and quality of life. 
SUPPLEMENTAL TABLE S1




Study details meta-analysis

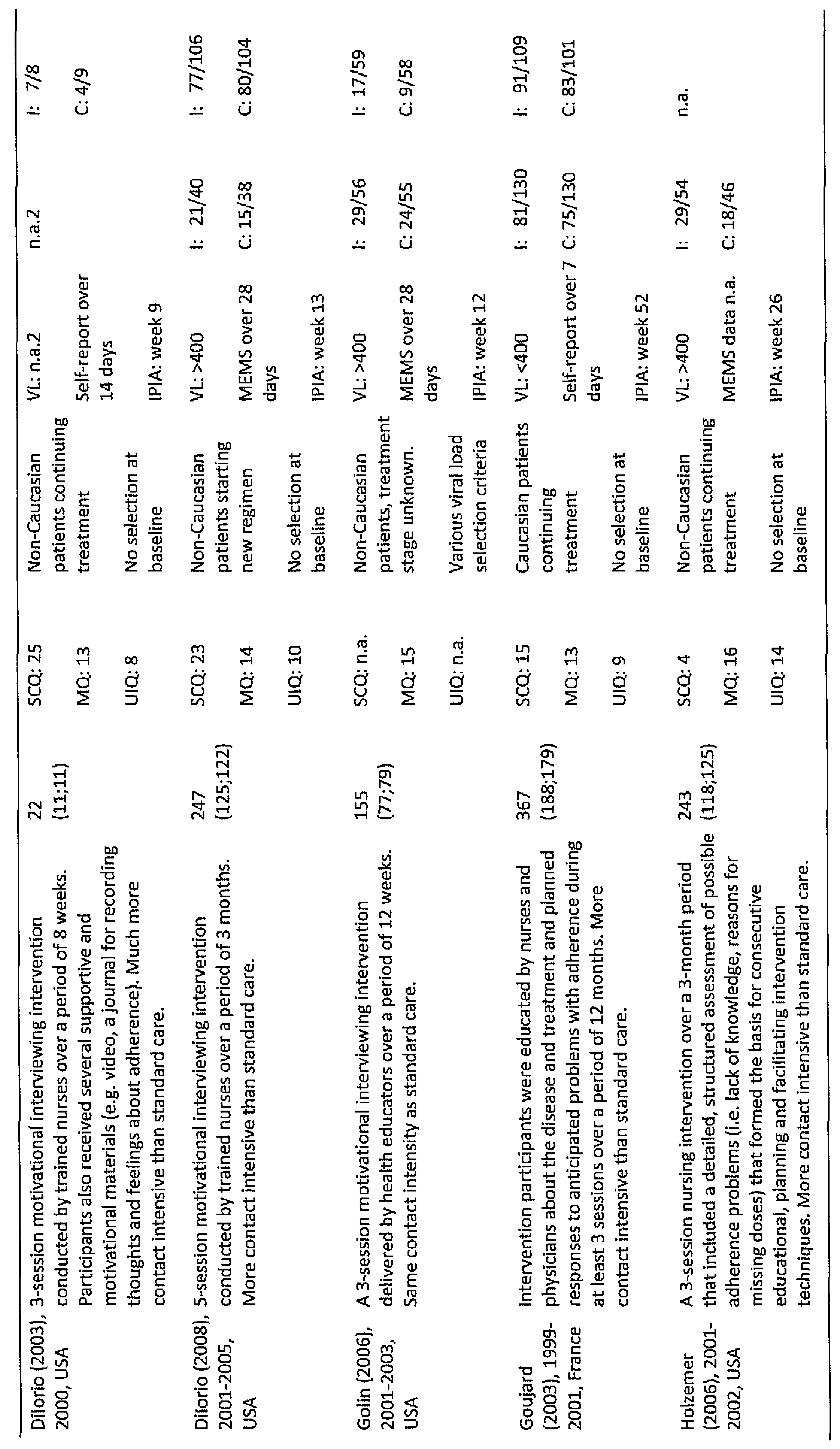


SUPPLEMENTAL TABLE S1

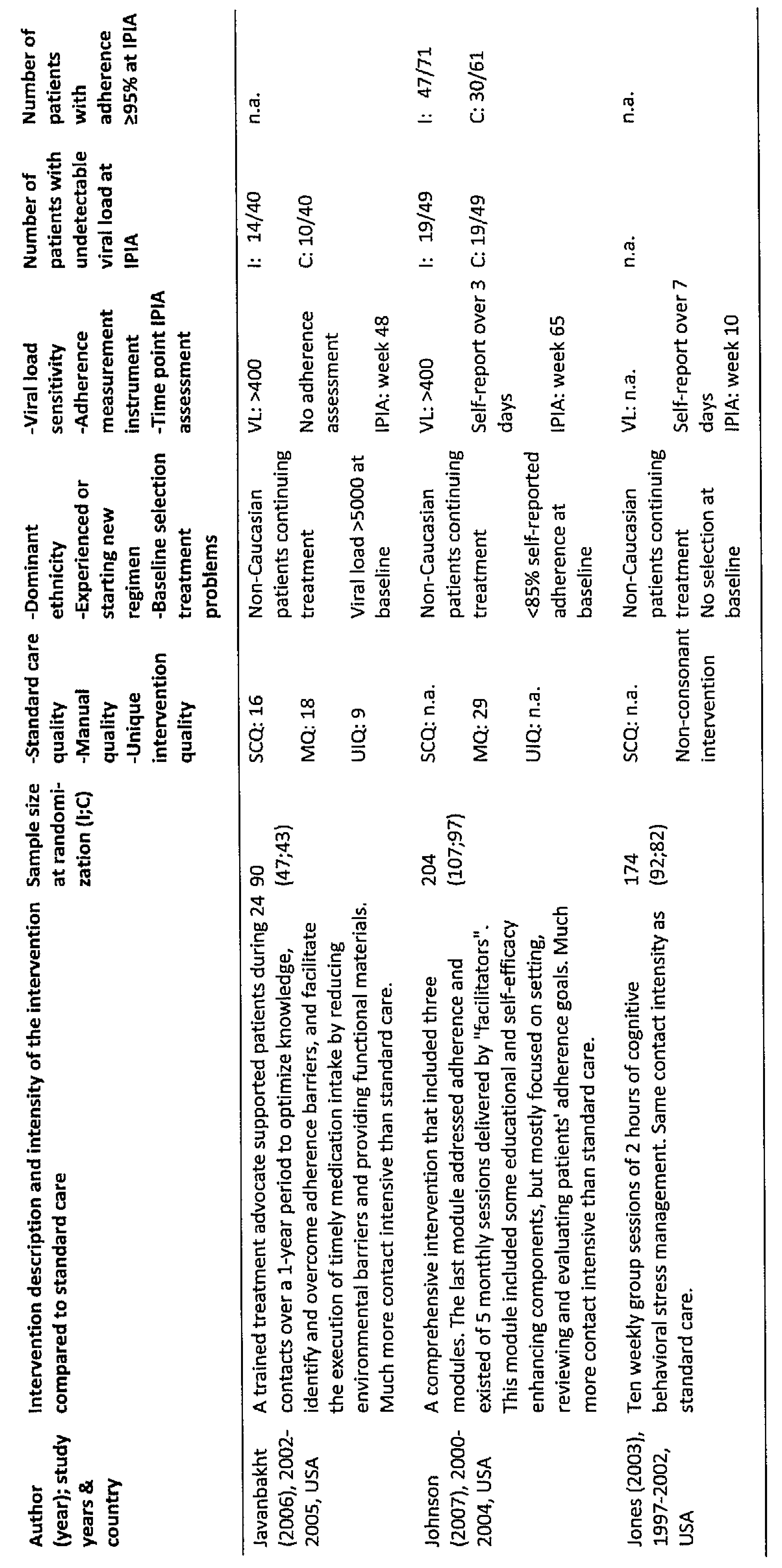


Study details meta-analysis

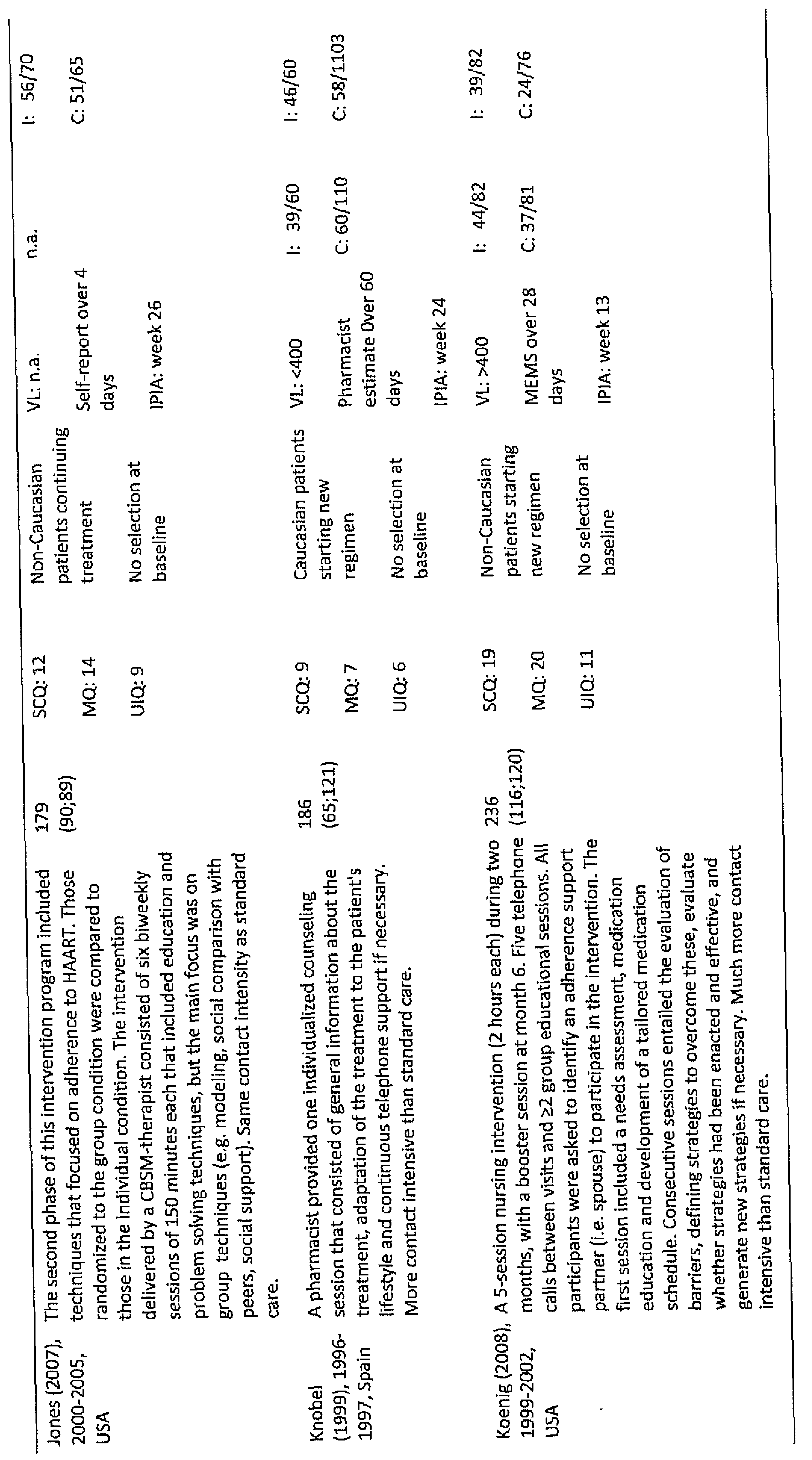


SUPPLEMENTAL TABLE S1

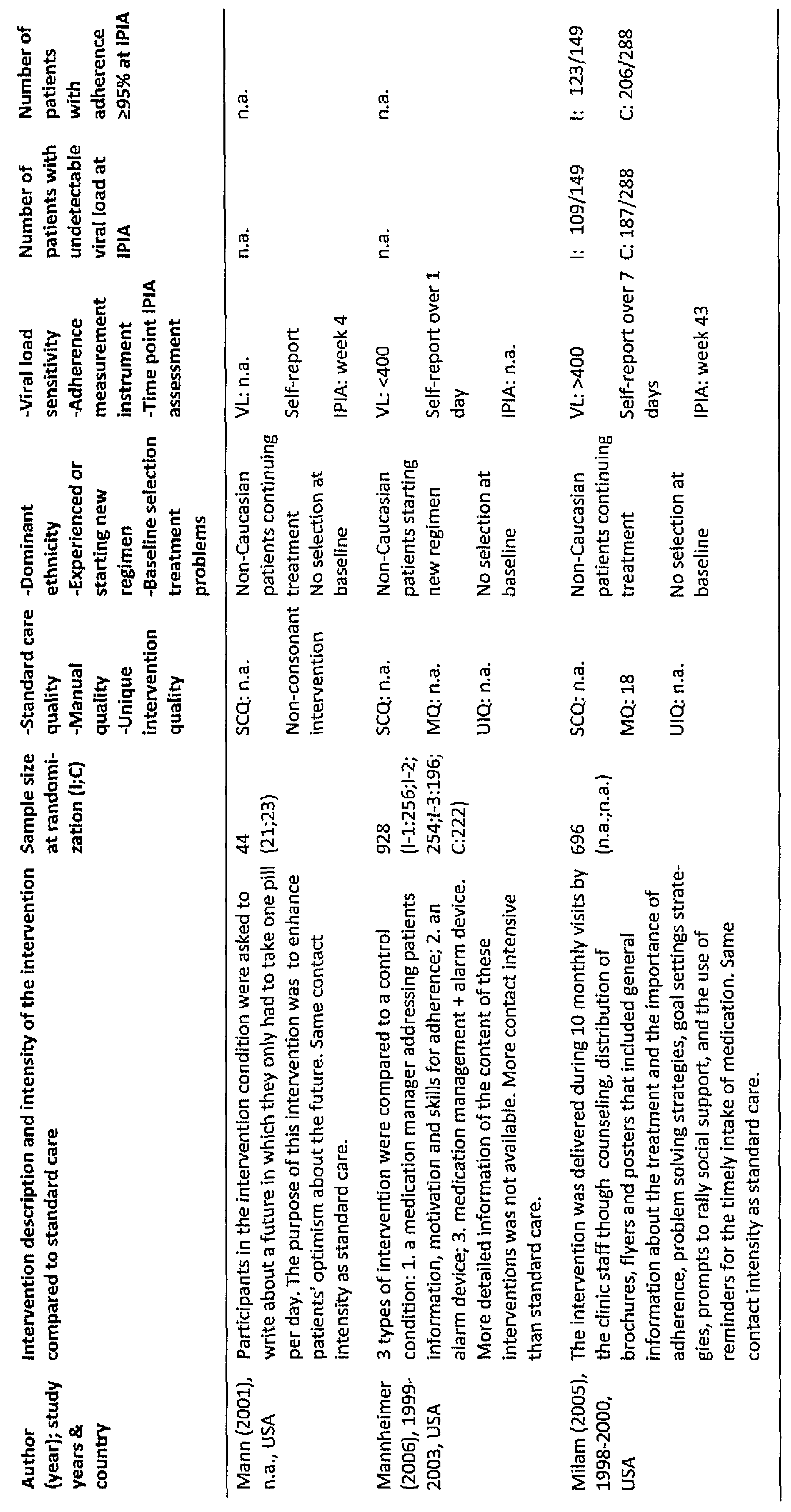


Study details meta-analysis

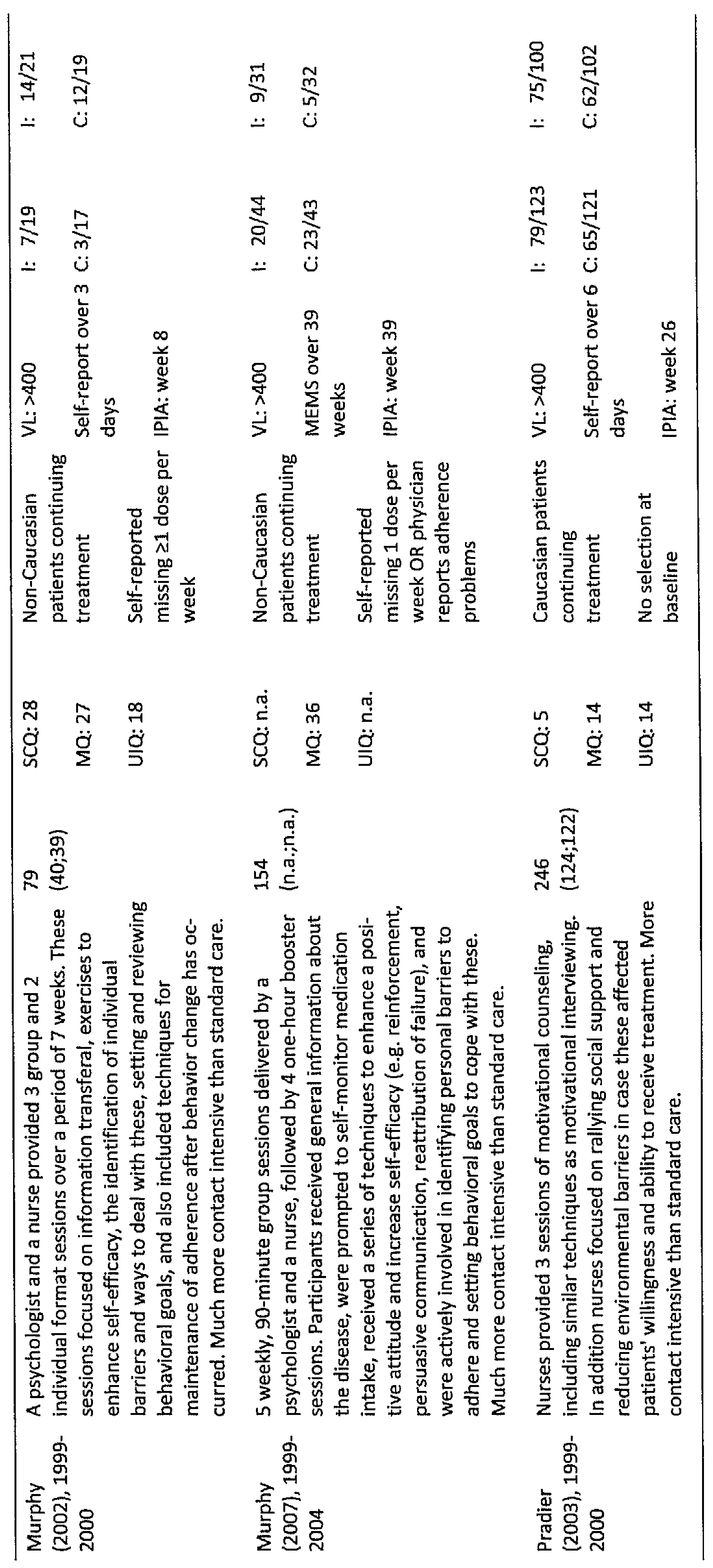


SUPPIEMENTAL TABLESI

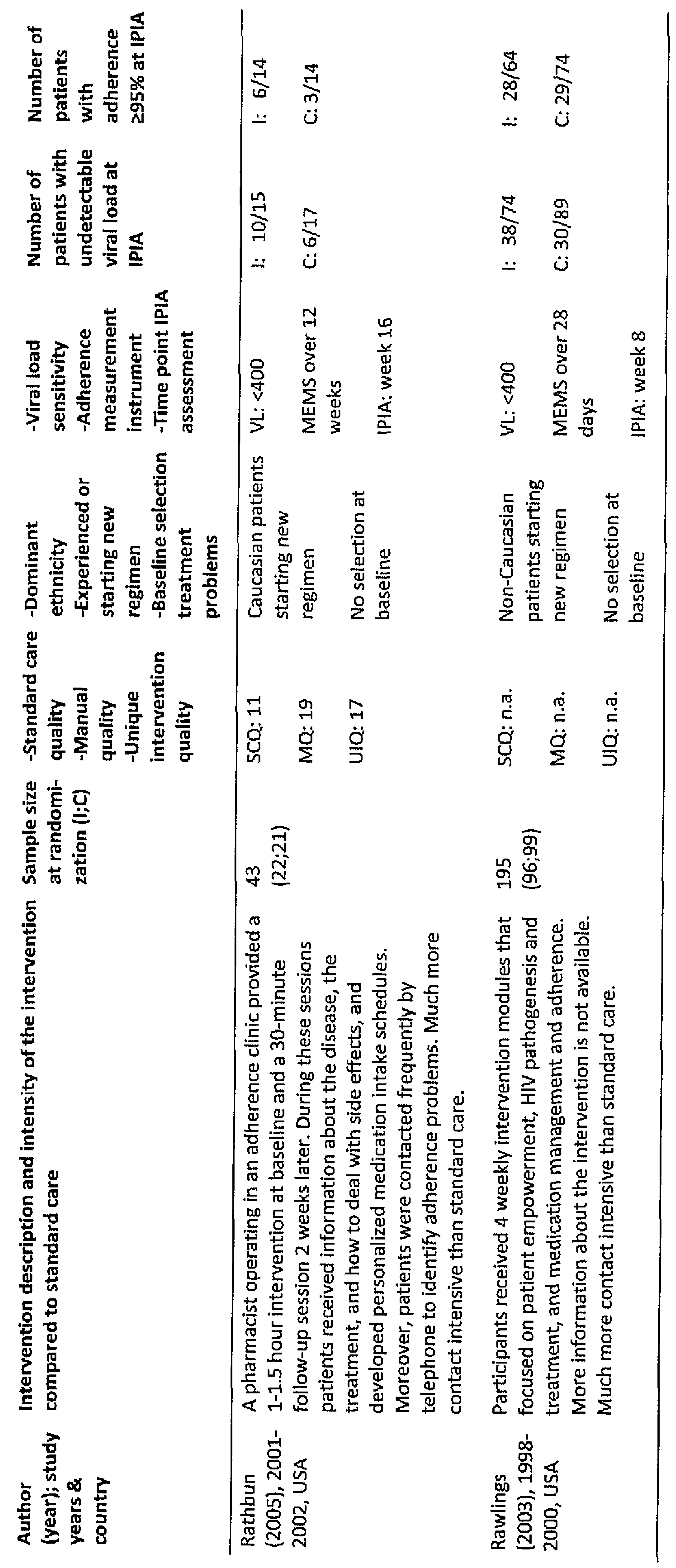

128 
Study details meta-analysis




SUPPLEMENTAL TABLE S1

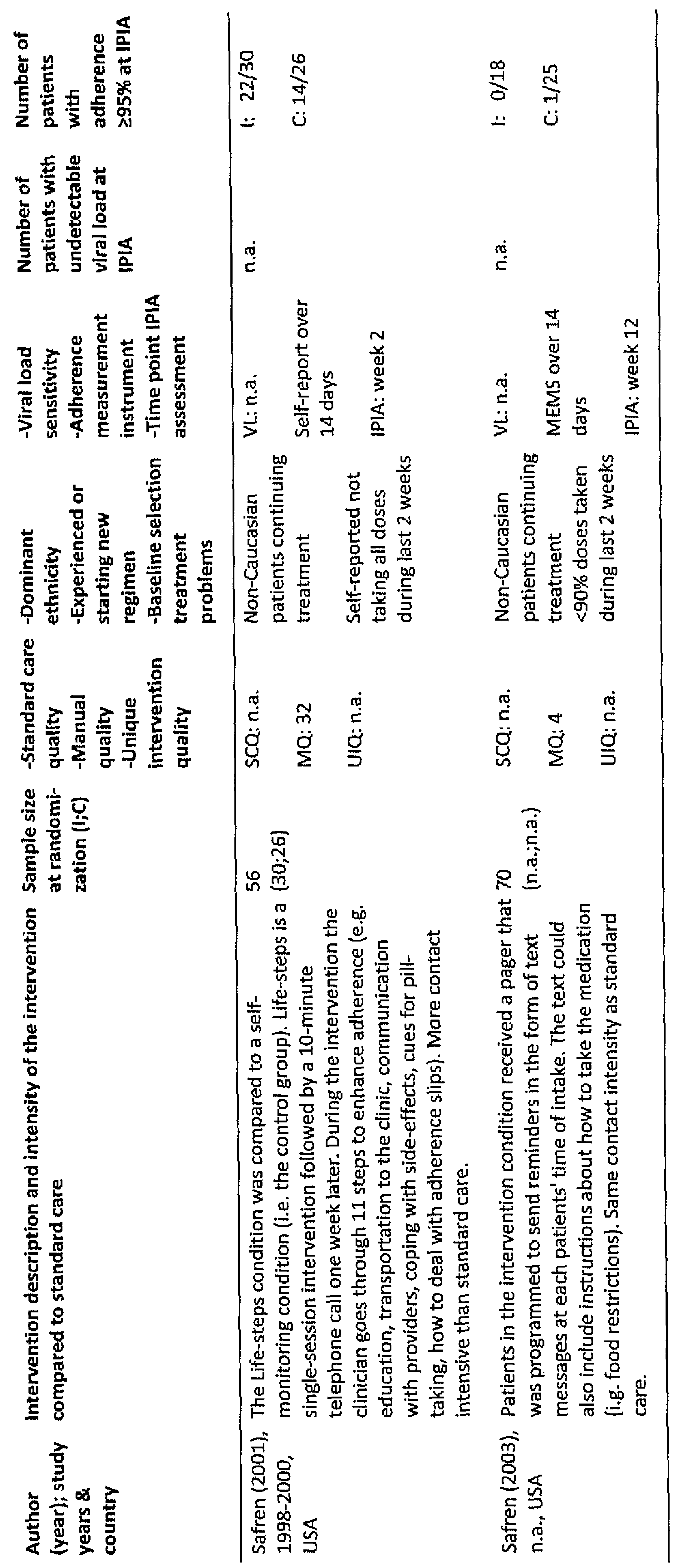

130 
Study details meta-analysis

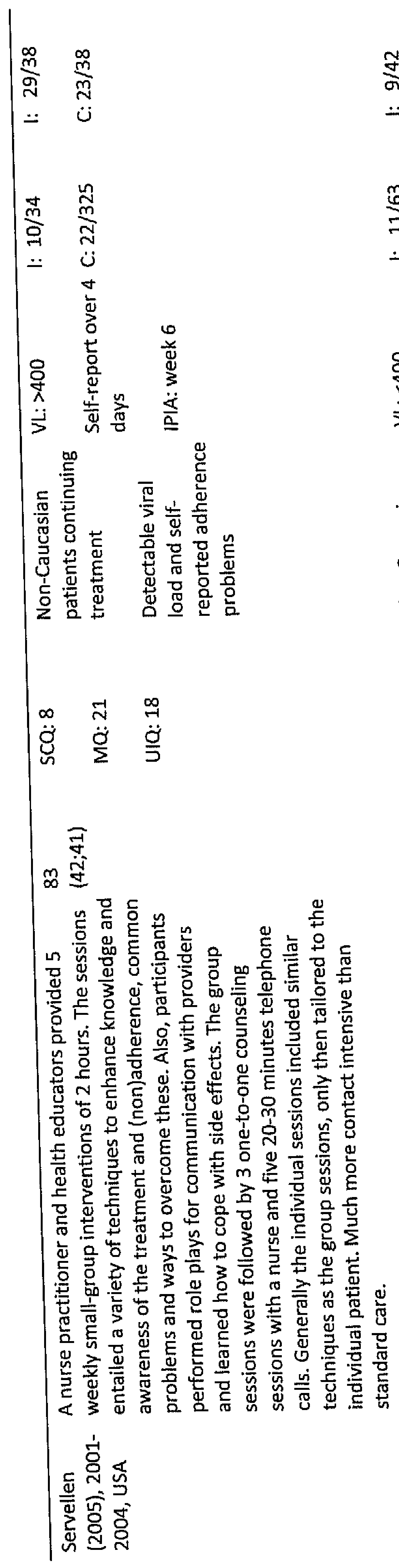

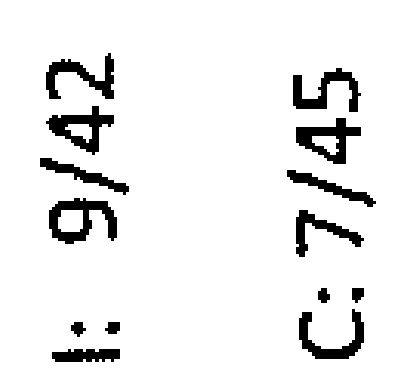

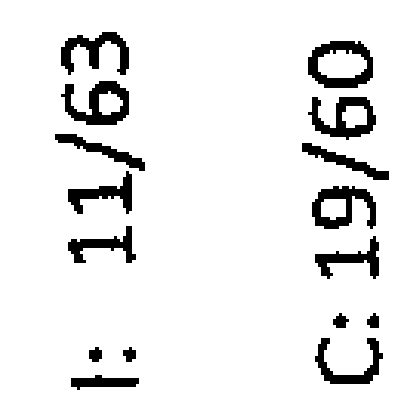

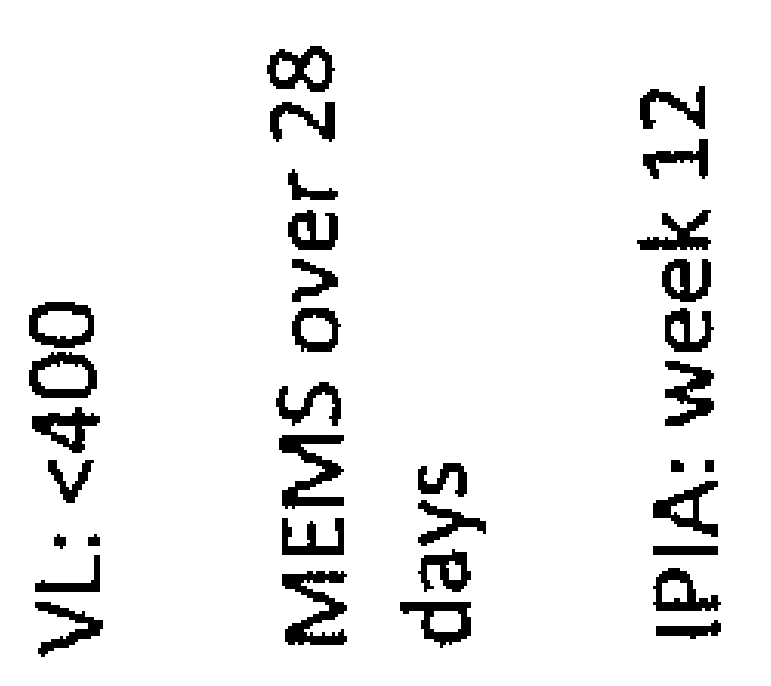

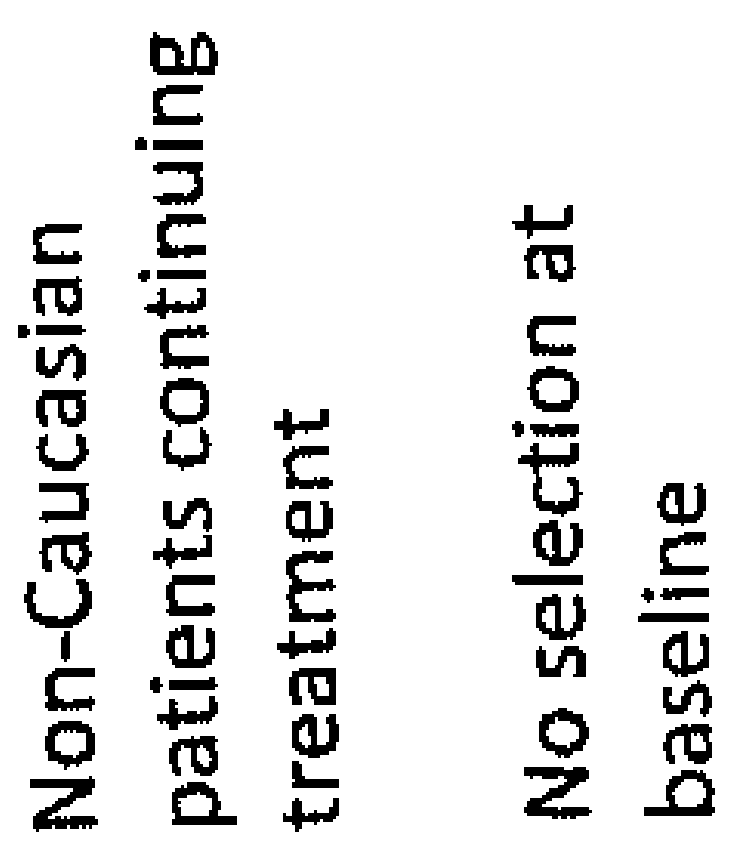

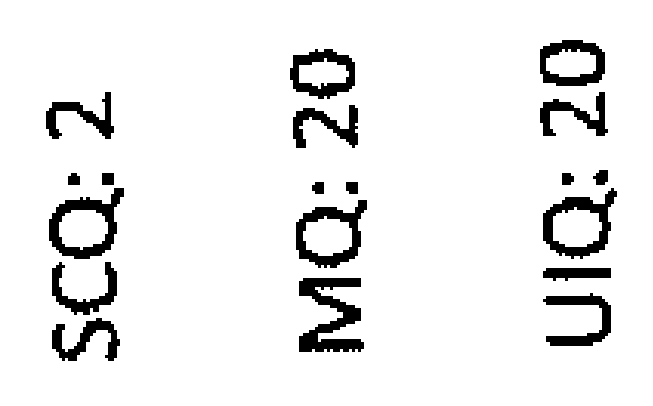

兽突

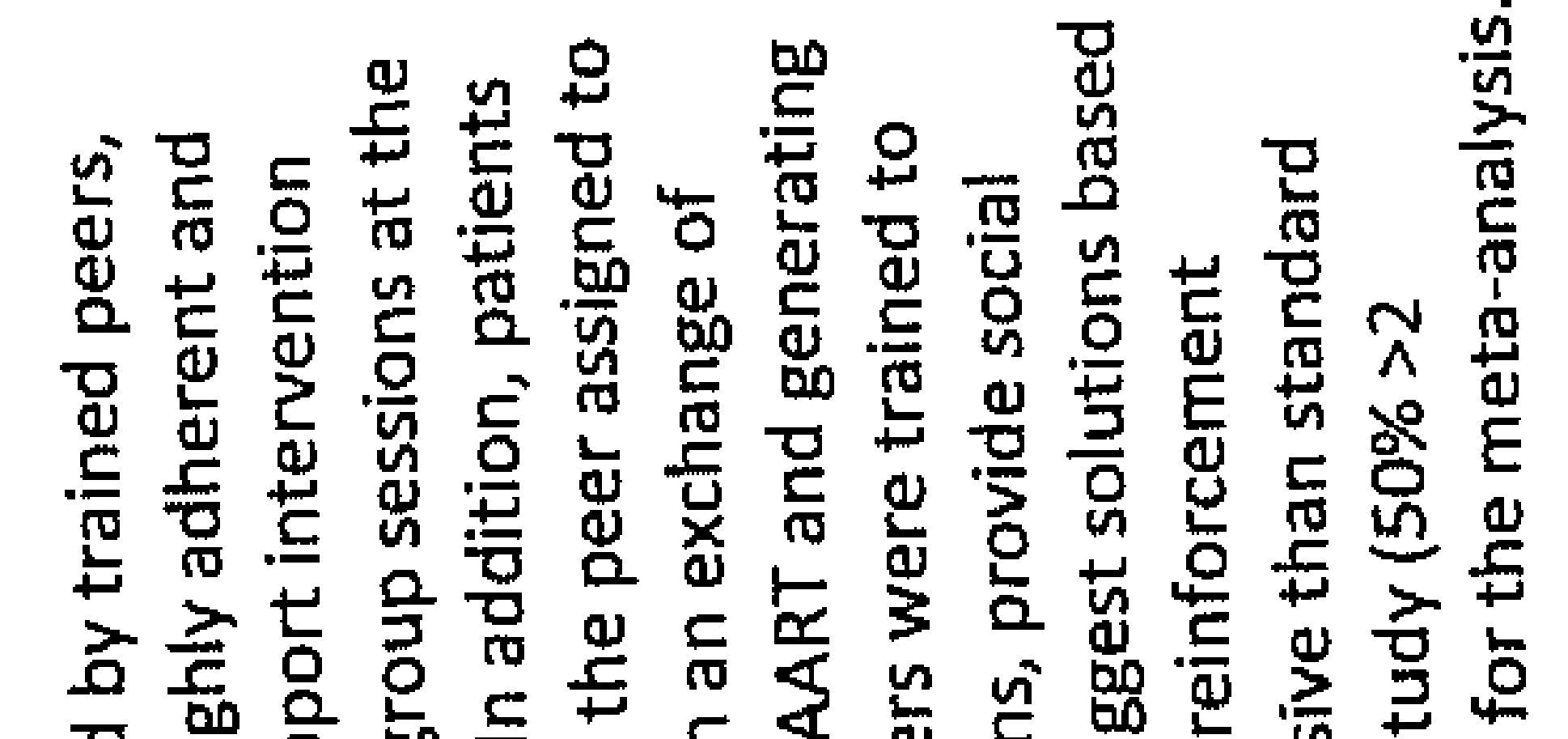

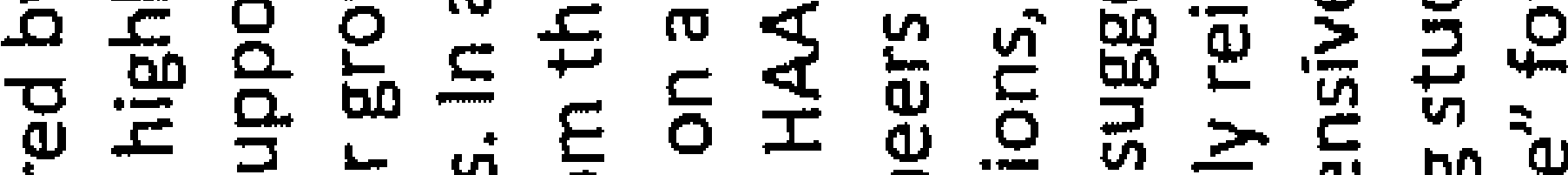

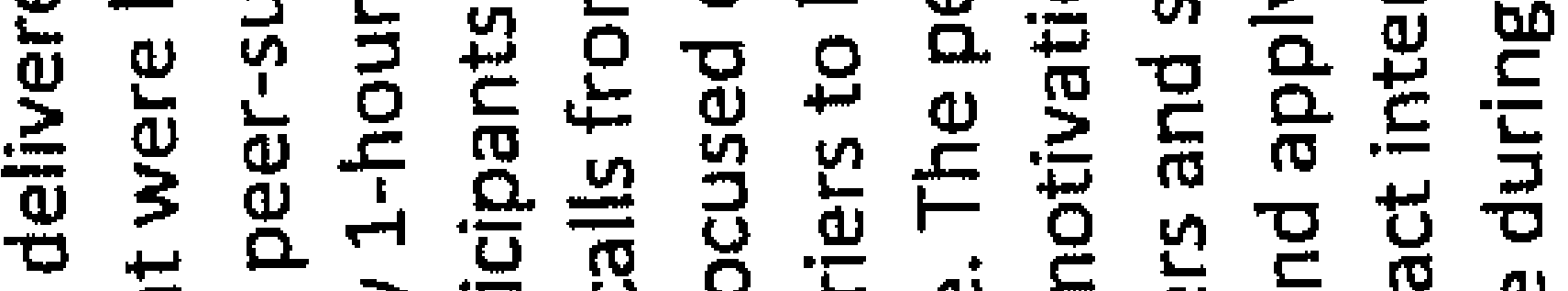

入

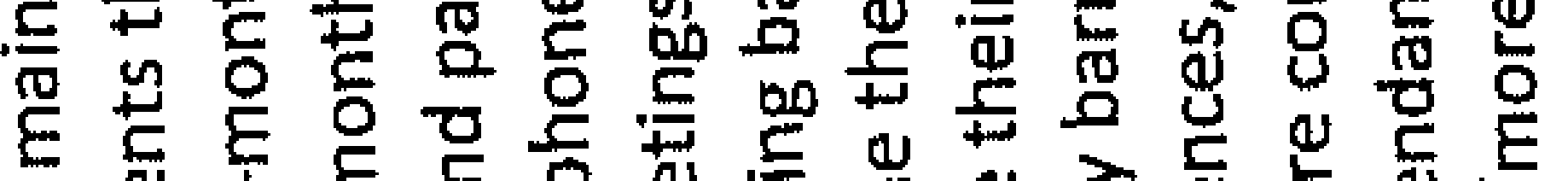

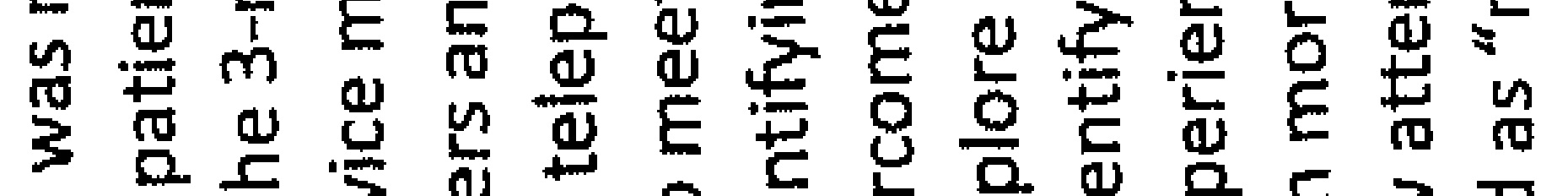

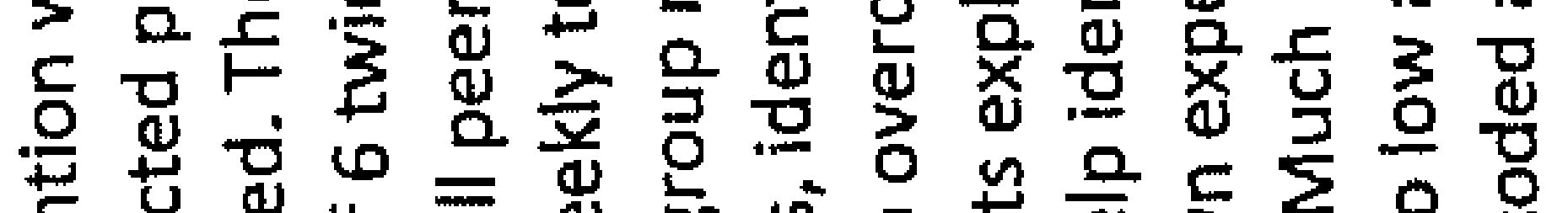

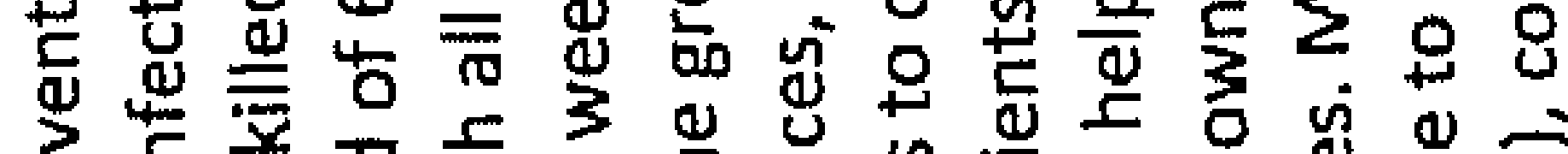

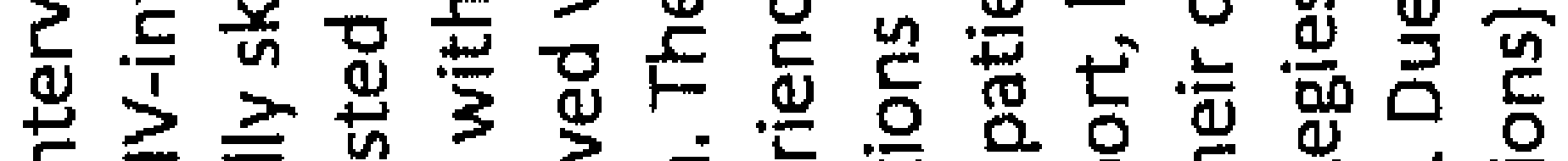

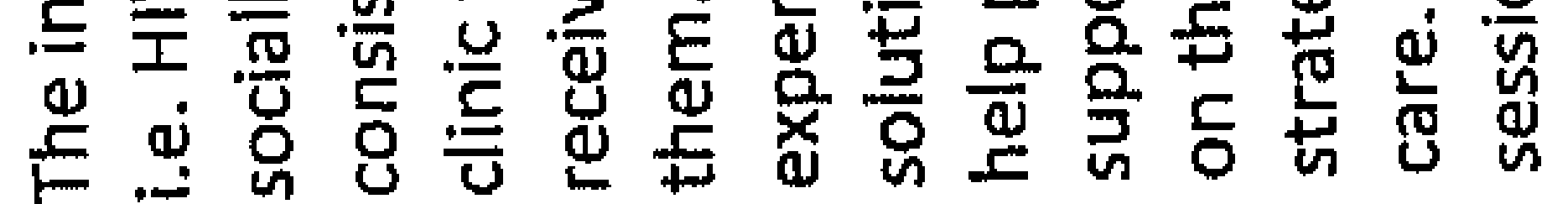

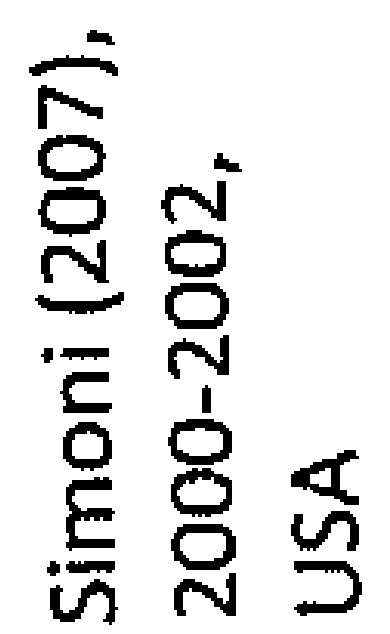


SUPPLEMENTAL TABLE $\$ 1$.

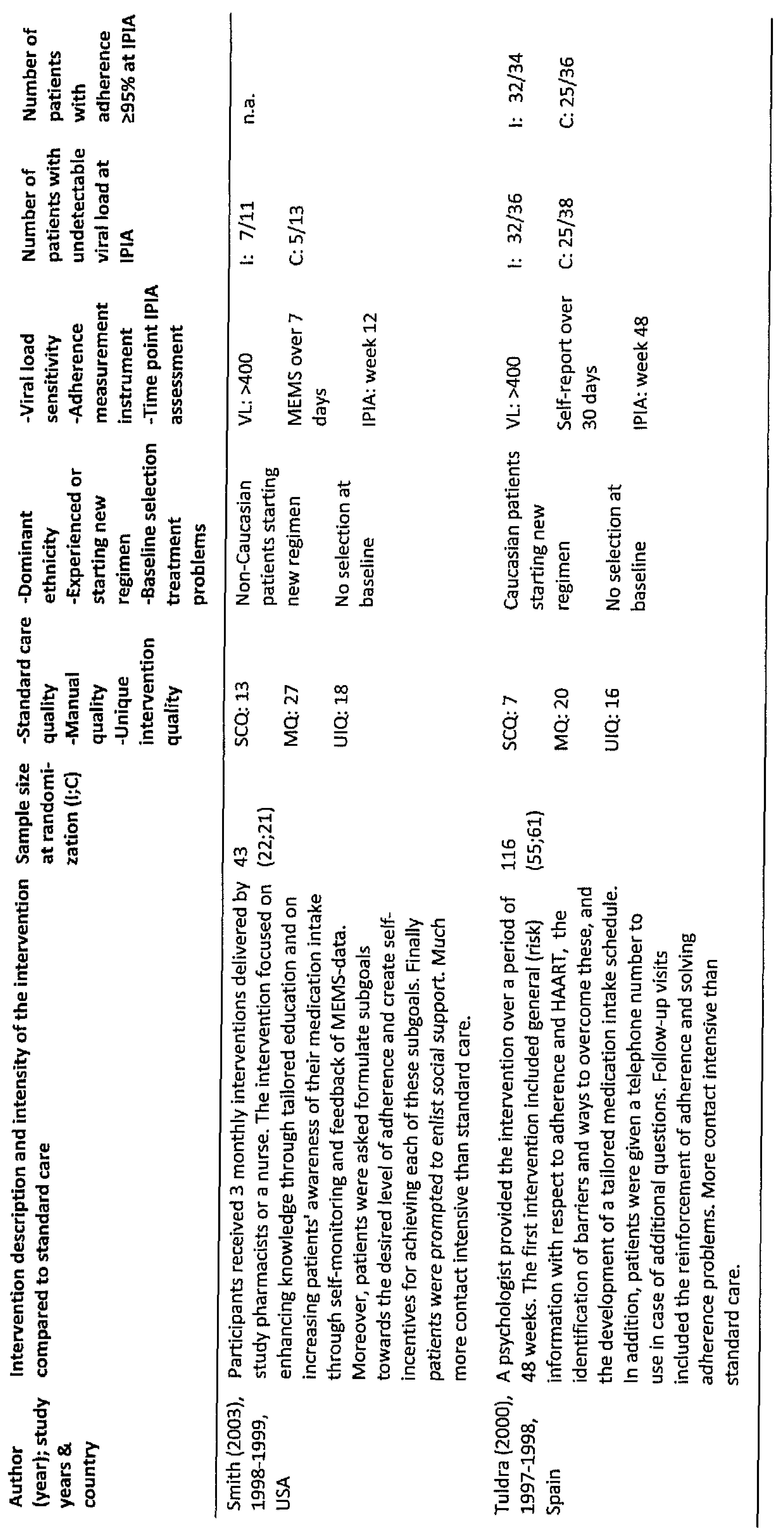


Study details meta-analysis

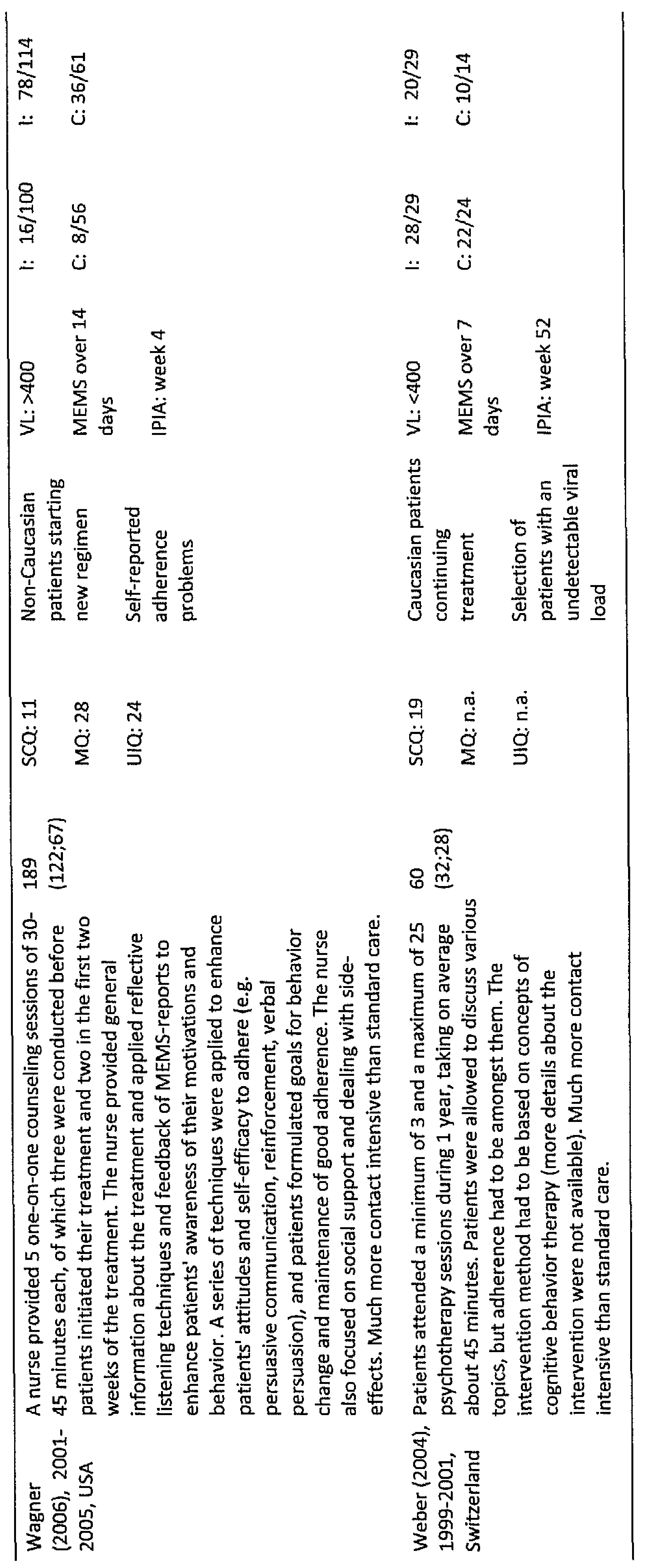


SUPPLEMENTAL TABLE SI

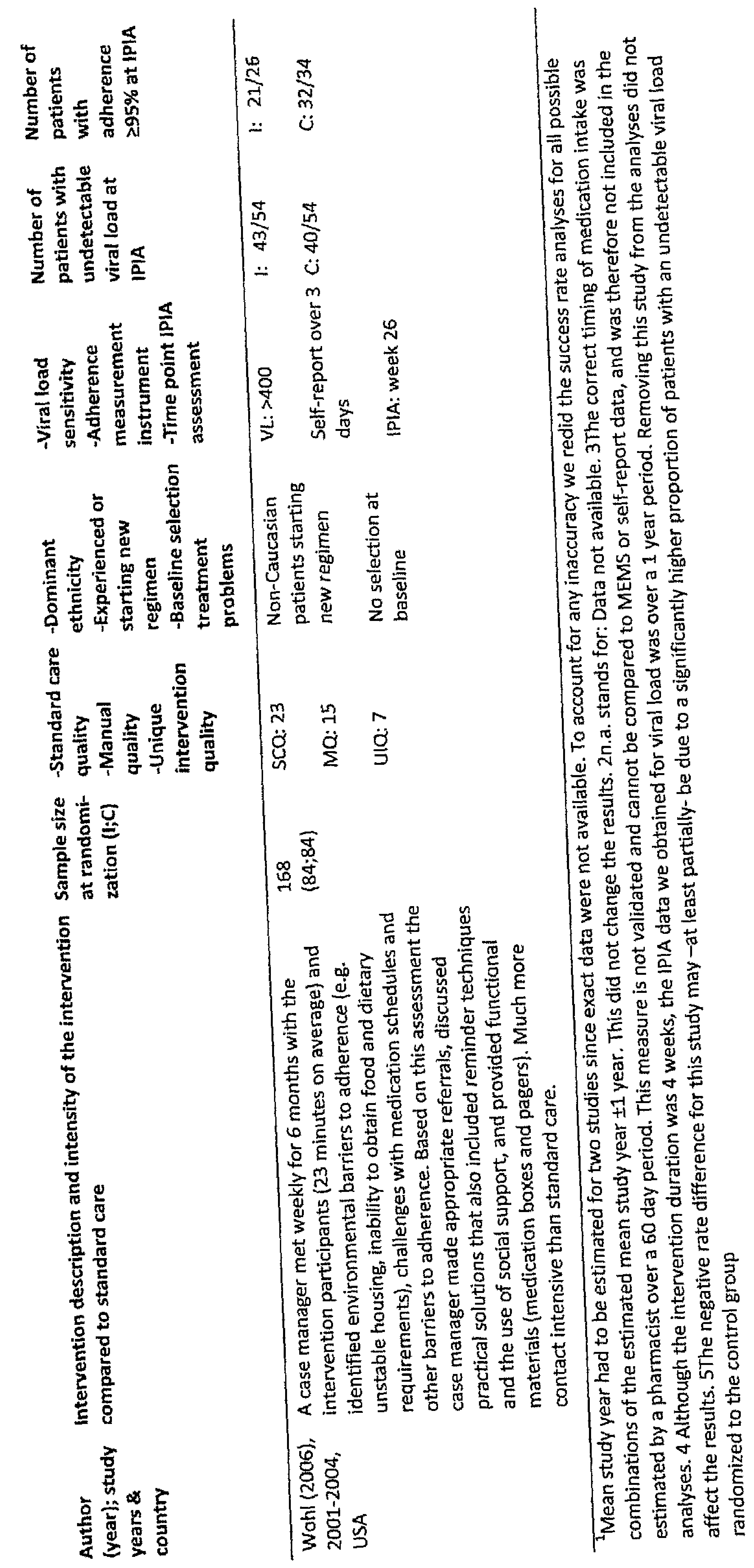




\section{REFERENCES}

Abraham, C., \& Michie, S. (2008). A taxonomy of behavior change techniques used in interventions. Health Psychology, 27(3), 379-387.

Acarturk, C., Cuijpers, P., van Straten, A., \& de Graaf, R. (2009). Psychological treatment of Social anxiety Disorder: a meta-analysis. Psychological Medicine, 39, 241-254.

Ajzen, I. (1988). Attitudes, personality and behavior. Milton Keynes: Open University Press.

Ajzen, I. (1991). The Theory of Planned Behavior. Organizational Behavior and Human Decision Processes, $50,179-211$.

Ajzen, I. (2002). Perceived behavioral control, self-efficacy, locus of control, and the Theory of Planned Behavior. Journal of Applied Social Psychology, 32(4), 665-683.

Albarracin, D., Johnson, B. T., Fishbein, M., \& Muellerleile, P. A. (2001). Theories of Reasoned Action and Planned Behavior as Models of condom use: A meta-analysis. Psychological Bulletin, 127(1), 142161.

Amico, K., Harman, J., \& O'Grady, M. (2008). Attrition and related trends in scientific rigor: A score card for ART adherence intervention research and recommendations for future directions. Current HIV/AIDS reports, 5, 172-185.

Amico, K. R., Harman, J. J., \& Johnson, B. T. (2006). Efficacy of antiretroviral therapy adherence interventions; a research synthesis of trials, 1996 to 2004. Journal of Acquired Immune Deficiency Syndromes, 41(3), 285-297.

Ammassari, A., Trotta, M. P., Murri, R., Castelli, F., Narciso, P., Noto, P., et al. (2002). Correlates and predictors of adherence to highly active antiretroviral therapy: overview of published literature. Journal of Acquired Immune Deficiency Syndromes 1999, 31 S3, S123-127.

Andrade, S. A., McGruder, H. F., Wu, A. W., Celano, S. A., Skolaski, R. L. Jr, Selnes, O. A., et al. (2005). A programmable prompting device improves adherence to Highly Active Antiretroviral Therapy in HIV-infected subjects with memory impairment. Clinical Infectious Diseases, 41, 875-882.

Antiretroviral cohort Collaboration. (2008). Life expectancy of individuals on combination antiretroviral therapy in high-income countries: a collaborative analysis of 14 cohort studies. Lancet, 26(372), 293-299.

Armitage, C. J., \& Conner, M. (1999). Distinguishing perceptions of control from self-efficacy: Predicting consumption of a low-fat diet using the theory of planned behavior. Journal of Applied Social Psychology, 29(1), 72-90.

Armitage, C. J., \& Conner, M. (2001). Efficacy of the theory of planned behaviour: A meta-analytic review. British Journal of Social Psychology, 40, 471 - 499.

Bagozzi, R. P. (1992). The self-regulation of attitudes, intentions, and behavior. Social Psychology Quarterly, 55(2), 178-204.

Bandura, A. (1997). Self-efficacy: The exercise of control: New York: W. H. Freeman.

Bangsberg, D. R., Charlebois, E. D., Grant, R. M., Holodniy, M., Deeks, S. G., Perry, S., et al. (2003). High levels of adherence do not prevent accumulation of HIV drug resistance mutations. AIDS, 17(13), 1925-1932.

Bartholomew, L. K., Parcel, G. S., Kok, G., \& Gottlieb, N. H. (2001). Intervention Mapping: Designing theory and evidence-based health promotion programs. Mountain View, CA: Mayfield Publishing Company.

Bartholomew, L. K., Parcel, G. S., Kok, G., \& Gottlieb, N. H. (2006). Planning health promotion programs: An Intervention Mapping approach (First edition ed.). San Fransisco: Jossey-Bass.

Bedell, S. E., Jabbour, S., Goldberg, R., Glaser, H., Gobble, S., Young-Xu, Y., et al. (2000). Discrepancies in the use of medications: their extent and predictors in an outpatient practice. Archives of Internal Medicine, 160, 2129-2134. 
Berger, S., Schad, T., von Wyl, V., Ehlert, U., Zellweger, C., Furrer, H., et al. (2008). Effects of cognitive behavioral stress management on HIV-1 RNA, CD4 cell counts and psychosocial parameters of HIVinfected persons. AIDS, 22, 767-775.

Bertholet, N., Favrat, B., Stubi, C. L. F., Brunner, H. R., \& Burnier, M. (2000). Why Objective Monitoring of Compliance is Important in the Management of Hypertension. Journal of Clinical Hypertension, II(IV), 258-262.

Biddle, S. J., \& Nigg, C. R. (2000). Theories of exercise behavior. International Journal of Sport Psychology, $31(2), 290-304$.

Boekaerts, M., Pintrich, P. R., \& Zeidner, M. (2000). Self-regulation: an introductory overview. In M. Boekaerts, P. R. Pintrich \& M. Zeidner (Eds.), Handbook of self-regulation (pp. 1-9). London: Academy Press.

Bond, W. S., \& Hussar, D. A. (1991). Detection methods and strategies for improving medication compliance. American Journal of Hospital Pharmacy, 48, 1978-1988.

Boutron, I., Moher, D., Altman, D., Schuiz, K., \& Ravaud, P. (2008). Extending the CONSORT statement to randomized trials of nonpharmacologic treatment: Explanation and elaboration. Annals of Internal Medicine, 148(4), 295-309.

Brian, A., Fisher, J. D., \& Fisher, W. A. (2002). Tests of the mediational role of preparatory safer sexual behavior in the context of the theory of planned behavior. Health Psychology, 21(1), 71-80.

Brug, J., Glanz, K., Van Assema, P., Kok, G., \& van Breukelen, G. (1998). The impact of computer-tailored feedback and iterative feedback on fat, fruit, and vegetable intake. Health Education and Behavior, 25, 517-531.

Collier, A., Ribaudo, H., Mukherjee, A., Feinberg, J., Fischl, M., \& Chesney, M. (2005). A Randomized study of serial telephone call support to increase adherence and thereby improve virologic outcome in persons initiating antiretroviral therapy. The Journal of Infectious Diseases, 192, 1398-1406.

Coté, J. K., \& Godin, G. (2005). Efficacy of interventions in improving adherence to antiretroviral therapy. International Journal of STD and AIDS, 16(5), 335-343.

Crespo-Fierro, M. (1997). Compliance/adherence and care management in HIV disease. Journal of the Association of Nurses in AIDS Care, 8(4), 43-54.

Davidson, K. W., Goldstein, M., Kaplan, R. M., Kaufmann, P. G., Knatterud, G. L., Orleans, C. T., et al. (2003). Evidence-Based Behavioral Medicine: What is It and How Do We Achieve It? Annals of Behavioral Medicine, 26(3), 161-171.

de Bruin, M., Hospers, H. J., Van Breukelen, G. J. P., Kok, G., Koevoets, W. M., \& Prins, J. M. (Submitted). Electronic monitoring-based counseling to enhance adherence and viral suppression among HIVinfected patients using HAART: A randomized controlled trial.

de Bruin, M., Hospers, H. J., van den Borne, H. W., Kok, G., \& Prins, J. M. (2005). Theory- and evidencebased intervention to improve adherence to antiretroviral therapy among HIV-infected patients in the Netherlands: A pilot study. AIDS Patient Care and STDs, 19(6), 384-394.

de Bruin, M., Viechtbauer, W., Hospers, H. J., Schaalma, H. P., \& Kok, G. (In press). Variability in standard care quality of HAART-adherence studies: Implications for the interpretation and comparison of intervention effects. Health Psychology.

DiClemente, C., \& Prochaska, J. (1998). Towards a comprehensive, transtheoretical model of change: Stages of change and addictive behaviors. In W. R. Miller \& N. Heather (Eds.), Treating addictive behaviors (pp. 3-24). New York: Plenum Press.

Dilorio, C., McCarty, F., Resnicow, M., McDonell Holstad, M., Soet, J., Yeager, K., et al. (2008). Using motivational interviewing to promote adherence to antiretroviral medications: A randomized controlled study. AIDS Care, 20(3), 273-283.

Dilorio, C., Resnicow, K., McDonell, M., Soet, J., McCarthy, F., \& Yeager, K. (2003). Using Motivational Interviewing to promote adherence to antiretroviral medications: A pilot study. Journal of the Association of Nurses in AIDS Care, 14(2), 52-65. 
Epstein, L. H., \& Cluss, P. A. (1982). A behavioral medicine perspective on adherence to long-term medical regimens. Journal of Consulting and Clinical Psychology, 50(6), 950-971.

EuroHIV. (2007). HIV/AIDS Surveillance in Europe. End-year report 2006.

Fairley, C. K., Levy, R., Rayner, C. R., Allardice, K., Costello, K., Thomas, C., et al. (2003). Randomized trial of an adherence programme for clients with HIV. International journal of STD and AIDS, 14, 805809.

Farmer, K. C. (1999). Methods for measuring and monitoring medication regimen adherence in clinical trials and clinical practice. Clinical Therapeutics, 21(6), 1074-1090.

Fennie, K. P., Bova, C. A., \& Williams, A. B. (2006). Adjusting and censoring electronic monitoring device data. Journal of Acquired Immune Deficiency Syndromes, 43(51), S88-595.

Fishbein, M., \& Ajzen, I. (1975). Belief, attitude, intention, and behavior. New York: Wiley.

Fisher, J., Fisher, W., Bryan, A., \& Misovich, S. (2002). Information-Motivation-Behavioral Skills Modelbased HIV risk behavior change intervention for inner-city high school youth. Health Psychology, 21(2), 177-186.

Fogarty, L., Roter, D., Larson, S., Burke, J., Gillespie, J., \& Levy, R. (2002). Patient adherence to HIV medication regimens: a review of published and abstract reports. Patient Education and Counseling, 46, 93-108.

Garcia, T., \& Pintrich, P. R. (1994). Regulating motivation and cognition in the classroom: the role of selfschemas and self-regulatory strategles. In D. H. Schunk \& B. J. Zimmerman (Eds.), Self-regulation of learning and performance: issues and educational applications (pp. 127-153). Hillsdale, NJ: Erlbaum.

Glanz, K., Rimer, B. K., \& Lewis, F. M. (2002). Health behavior and health education (3rd edition ed.). San Fransisco: Jossey-Bass.

Godin, G., Conner, M., \& Sheeran, P. (2005). Bridging the intention-behaviour 'gap': The role of moral norm. British Journal of Social Psychology, 44, 497-512.

Godin, G., Cote, J., Naccache, H., Lambert, L. D., \& Trottier, S. (2005). Prediction of adherence to antiretroviral therapy: a one-year longitudinal study. AIDS Care, 17(4), 493-504.

Godin, G., \& Kok, G. (1996). The Theory of Planned Behavior: A review of its applications to healthrelated behaviors. American Journal of Health Promotion, 11(2), 87-98.

Goldie, S. J., Paltiel, A. D., Weinstein, M. C., Losina, E., Seage, I. G., Kimmel, A. D., et al. (2003). Projecting the cost-effectiveness of adherence interventions in persons with Human Immunedeficiency Virus infection. American Journal of Medicine, 115, 632-641.

Golin, C., Earp, J., Tien, H., Stewart, P., Porter, C., \& Howie, L. (2006). A 2-arm, randomized, controlled trial of a motivational interviewing-based intervention to improve adherence to antiretroviral therapy (ART) among patients failing or initiating ART. Journal of Acquired Immune Deficiency Syndrome, 42, 42-51.

Golin, C. E., Liu, H., Hays, R. D., Miller, L. G., Beck, C. K., Ickovics, J., et al. (2002). A prospective study of predictors of adherence to combination antiretroviral medication. Journal of general internal medicine, 17(10), 756-765.

Gollwitzer, P. M. (1993). Goal achievement: The role of intentions. In W. Stroebe \& M. Hewstone (Eds.), European review of social psychology (pp. 141-185). Chichester, UK: Wiley.

Gollwitzer, P. M., \& Oettingen, G. (1998). The emergence and implementation of health goals. Psychology and Health, 13, 687-715.

Goujard, C., Bernard, N., Sohier, N., Peyramond, D., Lanc, F., Chwalow, J., et al. (2003). Impact of a patient education program on adherence to HIV medication: A randomized clinical trial. Journal of Acquired Immune Deficiency Syndrome, 34(2), 191-194.

Gras, L., van Sighem, A., Smit, C., Zaheri, S., Schuitemaker, H., \& de Wolf, H. (2008). Response to CART. Amsterdam: HIV Monitoring Foundation. 
Green, L. W., \& Kreuter, M. W. (1999). Health promotion planning: An educational and ecological approach (third edition ed.). CA.: Mayfield: Mountain View.

Gross, R., Yip, B., Lo Re III, V., Wood, E., Alexander, C. S., Harrigan, P. R., et al. (2006). A simple, dynamic measure of antiretroviral therapy adherence predicts failure to maintain HIV-1 suppression. The Journal of Infectious Diseases, 194, 1108-1114.

Haddad, M., Inch, C., Glazier, R. H., Wilkins, A. L., Urbshott, G. B., Bayoumi, A., et al. (2000). Patient support and education for promoting adherence to highly active antiretroviral therapy for HIV/AIDS. (Vol. 4). Cochrance Database of Systematic Reviews, 3, CD001442.

Halkitis, P., Palamar, J., \& Mukherjee, P. (2008). Analysis of HIV medication adherence in relation to person and treatment characteristics using hierarchical limear modeling. AlDS Patient Care and STDs, 22(4), 323-335.

Hall, J. A., Roter, D. L., \& Katz, L. R. (1988). Meta-analysis of provider behavior in medical encounters. Medical Care, 26, 657-675.

Haynes, R., Yao, X., Degani, A., Kripalani, S., Garg, A., \& McDonald, H. (2005). Interventions to enhance medication adherence. Cochrance Database of Systematic Reviews, 4, CD000011.

Haynes, R. B., Ackloo, E., Sahota, N., McDonald, H. P., \& Yao, X. (2008). Interventions for enhancing medication adherence. Cochrane Database of Systematic Reviews, 2, CD00011.

Haynes, R. B., McDonald, H., Garg, A. X., \& Montague, P. (2002). Interventions for helping patients to follow prescriptions for medication. The Cochrane Database of Systematic Reviews, 2, CD000011.

Haynes, R. B., McKibbon, K. A., \& Kanani, R. (1996). Systematic review of randomized trials of interventions to assist patients to follow prescriptions for medications. The Lancet, 348, 383-386.

Higgins, J. P. T., \& Green, S. (2005). Introduction: Systematic reviews and the Cochrane Handbook. In J. P. T. Higgins \& S. Green (Eds.), Cochrane Handbook for Systematic Reviews of Interventions 4.2.5 [updated May 2005]; Section 8. Chichester, UK: John Wiley \& Sons, Ltd,

Hogg, R., Heath, K., Bangsberg, D., Yip, B., Press, N., O'Shaugnessy, M., et al. (2002). Intermittent use of tripple-therapy is predictive of mortality at baseline and after 1 year of follow-up. AIDS, 16(7), 1051-1058.

Holzemer, W. L., Bakken, S., Portillo, C. J., Grimes, R., Welch, J., Wantland, D., et al. (2006). Testing a Nurse-Tailored HIV Medication Adherence Intervention. Nursing research, 55(3), 189-197.

Howard, A. A., Arnsten, J. H., Lo, Y., Vlahov, D., Rich, J. D., Schuman, P., et al. (2002). A prospective study of adherence and viral load in a large multi-center cohort of HIV-infected women. AIDS, 16, 2175 2182.

Hugen, P. W. H., Langebeek, N., Burger, D. M., Zomer, B., Leusem, R. V., Schuurman, R., et al. (200Z). Assessment of adherence to HIV protease inhibitors: comparison and combination of various methods, including MEMS (electronic monitoring), patient and nurse report, and therapeutic drug monitoring. Journal of Acquired Immune Deficiency Syndromes, 30, 324-334.

Ickovics, J. R., \& Meade, C. S. (2002). Adherence to HAART among patients with HIV: breakthroughs and barriers. AlOS Care, 14, 309-318.

Ismail, K., Winkley, K., \& Rabe-Hesketh, S. (2004). Systematic review and meta-analysis of randomized controlled trials of psychological interventions to improve glycaemic control in patients with type 2 diabetes. The Lancet, 363, 1589-1597.

Javanbakht, M., Prosser, P., Grimes, T., Weinstein, M., \& Farthing, C. (2006). Efficacy of an individualized adherence support program with contingent reinforcement among nonadherent HIV-positive patients. Journal of the International Association of Physician in AIDS Care, 5(4), 143-150.

Johnson, M., Charlebois, E., Morin, S., Remien, R., \& Chesney, M. (2007). Effects of a behavioral intervention on Antiretroviral medication adherence among people living with HIV: The Healthy Living Project randomized controlled study. Journal of Acquired Immune Deficiency Syndrome, $46(5), 574-580$. 
Joined United Nations Programme on HIV/AIDS. (2007). AIDS epidemic update: December 2007. Joined United Nations Programme on HIV/AIDS. (2008). Report on the global AIDS epidemic.

Jones, D., Ishii, M., LaPerriere, A., Stanley, H., Antoni, M., Ironson, G., et al. (2003). Influencing medication adherence among women with AIDS. AIDS CARE, 15(4), 463-474.

Jones, D., McPherson-Baker, S., Lydston, D., Camille, J., Brondolo, E., Tobin, J., et al. (2007). Efficacy of a group medication adherence intervention among HIV positive women: The SMARTEST Women's Project. AIDS and Behavior, 11, 79-86.

Kastrissios, H., \& Blaschke, T. F. (1997). Medication compliance as a feature in drug development. Annual Review of Pharmacology and Toxicology, 37, 451-475.

Knobel, H., Alonso, J., Casado, J. L., Collazos, J., Gonzalez, J., Ruiz, I., et al. (2002). Validation of a simplified medication adherence questionnaire in a large cohort of HIV.infected patients: the GEEMA Study. AIDS, 16(4), 605-613.

Knobel, H., Carmona, A., Lopez, J. L., Gimeno, J. L., Saballs, P., Gonzalez, A., et al. (1999). Adherence to highly active antiretroviral treatment: impact of individualized assessment [Adherencia al tratamiento antirretroviral de gran actividad: impacto de una intervencion de asesoramiento individualizado]. Efermedades Infecciocas Microbiologica y Clinica, 17, 78-81.

Knobel, H., Guelar, A., Carmona, A., Espona, M., González, A., López-Colomés, J. L., et al. (2001). Virologic outcome and predictors of virologic failure of Highly Active Antiretroviral Therapy containing Protease Inhibitors. AIDS Patient Care and STDs, 15, 193-199.

Koenig, L., Pals, S., Bush, T., Palmore, M., Stratford, D., \& Ellerbrock, T. (2008). Randomized controlled trial of an intervention to prevent adherence failure among HIV-infected patients initiating Antiretroviral therapy. Health Psychology, 27(2), 159-169.

Levy, R. W., Rayner, C. R., Fairley, C. K., Kong, D. C., Mijch, A., Costello, K., et al. (2004). Multidisciplinary HIV adherence intervention: a randomized study. AIDS Patient Care and STDs, 18(12), 728-735.

Liu, H., Golin, C. E., Miller, L. G., Hays, R. D., Beck, C. K., Sanandaij, S., et al. (2001). A comparison study of multiple measures of adherence to HIV protease inhibitors. Annals of Internal Medicine, 134, 968977.

Liu, H., Miller, L. G., Hays, R. D., Golin, C. E., Wu, T., Wenger, N. S., et al. (2006). Repeated measures longitudinal analyses of HIV virologic response as a function of percent adherence, dose timing, genotypic sensitivity, and other factors. Journal of Acquired Immune Deficiency Syndromes, 41(3), 315-322.

Mackinnon, D. P., Lockwood, C. M., Hoffman, J. M., West, S. G., \& Sheets, V. (2002). A comparison of methods to test mediation and other intervening variable effects. Psychological Methods, 7(1), 83104.

Mann, T. (2001). Effects of future writing and optimism on health behaviors in HIV-infected women. Annals of Behavioral Medicine, 23(1), 26-33.

Mannheimer, S., Morse, E. M., JP, Andrews, L., Child, C., Schmetter, B., \& Friedland, G. (2006). Sustained benefit from a long-term Antiretroviral adherence intervention results of a large randomized clinical trial. Journal of Acquired Immune Deficiency Syndrome, 43(S1), 41-47.

Mazzuca, S. A. (1982). Does patient education in chronic disease have therapeutic value? Journal of Chronic Diseases, 35, 521-529.

McDonald, H. P., Garg, A. X., \& Haynes, R. B. (2002). Interventions to enhance patient adherence to medication prescriptions: scientific review. JAMA, 288(22), 2868-2879.

McNabb, J., Ross, J. W., Abriola, K., Turley, C., Nightingale, C. H., \& Nicolau, D. P. (2001). Adherence to Highly Active Antiretroviral Therapy predicts virologic outcome at an inner-city Human Immunedeficiency Virus clinic. Clinical Infectious Diseases, 33, 700-705.

McPherson-Baker, S., Malow, R., Penedo, F., Jones, D., Schneiderman, N., \& Klimas, N. (2000). Enhancing adherence to combination antiretroviral therapy in non-adherent HIV-positive men. AIDS Care, 12(4), 399-404. 
Melbourne, K., Geletko, S., Brown, S., Willey-Lessne, C., Chase, S., \& Fisher, A. (1999). Medication adherence in patients with HIV infection: A comparison of two measurement methods. Aids Reader, 9(5), 329-338.

Milam, J., Richardson, J. L., McCutchan, A., Stoyanoff, S., Weiss, J., Kemper, C., et al. (2005). Effect of a brief antiretroviral adherence intervention delivered by HIV care providers. Journal of Acquired Immune Deficiency Syndromes, 40(3), 356-363.

Miller, L. G., \& Hays, R. D. (2000). Measuring adherence to antiretroviral medications in clinical trials. HIV Clinical Trials, 1(1), 36-46.

Mocroft, A., Katlama, C., Johnson, A., Pradier, C., Antunes, F., Mulcahy, F., et al. (2000). AIDS across Europe, 1994-98: the EuroSIDA study. Lancet, 356, 291-296.

Moore, R., \& Chaisson, R. (1999). Natural history of HIV infection in the era of combination antiretroviral therapy. AIDS, 13(14), 1933-1942.

Mullen, P. D., Green, L. W., \& Persinger, G. S. (1985). Clinical trials of patient education for chronic conditions: A comparative meta-analysis of intervention types. Preventive Medicine, 14, 753-781.

Murphy, D., Lu, M., Martin, D., Hoffman, D., \& Marelich, W. (2002). Results of a pilot intervention trial to improve Antiretroviral adherence among HIV-positive patients. Journal of the Association of Nurses in AIDS Care, 13(6), 57-69.

Murphy, D., Marelich, W., Rappaport, N., Hoffman, D., \& Farthing, C. (2007). Results of an Antiretroviral adherence intervention: STAR (Staying Healthy: Taking Antiretrovirals Regularly). Journal of the International Association of Physician in AIDS Care, 6(2), 113-124.

Nellen, J. F. J. B., Wit, F. W., Wolf, F. d., Jurriaans, S., Lange, J. M., \& Prins, J. M. (2004). Virological and immunological response to HAART in indigenous and non-indigenous HIV-1 infected patients in the Netherlands: a cohort study. Journal of Acquired Immune Deficiency Syndrome, 36, 943-950.

Nieuwkerk, P. T., Sprangers, M. A., Burger, D. M., Hoetelmans, R. M., Hugen, P. W., Danner, S. A., et al. (2001). Limited patient adherence to highly active antiretroviral therapy for HIV-1 infection in an observational cohort study. Archives of internal medicine, 161(16), 1962-1968.

Noar, S., Benac, C., \& Harris, M. (2007). Does tailoring matter? Meta-analytic review of tailored print health behavior change interventions. Psychological Bulletin, 133(4), 673-693.

Norman, P., Sheeran, P., \& Orbell, S. (2003). Does state versus action orientation moderate the intentionbehavior relationship? Journal of Applied Social Psychology, 33(3), 536-553.

Osterberg, L., \& Blaschke, T. (2005). Adherence to Medication. New England Journal of Medicine, 353(5), 487-497.

Ouellette, J. A., \& Wood, W. (1998). Habit and Intention in everyday life: the multiple processes by which past behavior predicts future behavior. Psychological Bulletin, 124, 54-74.

Paterson, D. L., Potoski, B., \& Capitano, B. (2002). Measurement of adherence to antiretroviral medications. Journal of Acquired Immune Deficiency Syndromes, 31 Suppl 3, S103-106.

Paterson, D. L., Swindells, S., Mohr, J., Brester, M., Vergis, E. N., Squier, C., et al. (2000). Adherence to protease inhibitor therapy and outcomes in patients with HIV infection. Annals of Internal Medicine, 133(1), 21-30.

Perno, C. F., Ceccherini Silberstein, F., De Luca, A., Cozzi Lepri, A., Gori, C., Cingolani, A., et al. (2002). Virologic correlates of adherence to antiretroviral medications and therapeutic failure. Journal of Acquired Immune Deficiency Syndromes 1999, 31 (53), S118-122.

Peters, G.-J. Y., Kok, G., \& Abraham, C. (2008). Social cognitive determinants of ecstasy use to target in evidence-based interventions: A meta- analytical review. Addiction, 103(1), 109-118.

Peterson, G. M., McLean, S., \& Millingen, K. S. (1984). A randomized trial to improve patient compliance with anticonvulsant therapy. Epilepsia, 25(4), 412-417. 
Petty, R., Barden, J., \& Wheeler, S. (2002). The elaboration likelihood model of persuasion: Health promotions that yield sustained behavioral change. In R. DiClemente, R. Crosby \& M. Kegler (Eds.), Emerging theories in health promotion practice and research (pp. 71-99). San Fransisco: JosseyBass.

Posovac, E. J. (1980). Evaluations of patient education programs: a meta-analysis. Evaluation \& the Health Professions, 3(1), 47-62.

Posovac, E. J., Sinacore, J. M., Brotherton, S. E., Helford, M. C., \& Turpin, R. S. (1985). Increasing compliance to medical treatment regimen: a meta-analysis of program evaluation. Evaluation \& the Health Professions, 8(1), 7-22.

Pradier, c., Bentz, 1., Spire, B., Tourette-Turgis, C., Morin, M., Souville, M., et al. (2003). Efficacy of an educational and counseling intervention on adherence to Highly Active Antiretroviral Therapy: French prospective controlled study. HIV Clinical Trials, 4(2), 121-131.

Preacher, K. J., \& Hayes, A. F. (2008). Asymptotic and resampling strategies for assessing and comparing indirect effects in multiple mediator models. Behavior Research Methods, 40(3), 879-891.

Prendergast, M., Podus, D., Chang, E., \& Urada, D. (2002). The effectiveness of drug abuse treatment: a meta-analysis of comparison group studies. Drug and Alcohol Dependence, 67, 53-72.

Prochaska, J., DiClemente, C., \& Norcross, J. (1997). In search of how people change: Applications to addictive behaviors. In G. Marlatt \& G. VandenBos (Eds.), Addictive behaviors: Readings on etiology, prevention, and treatment (pp. 671-696). Washington, DC: American Psychological Association.

Prochaska, J. O., \& DiClemente, C. C. (1983). Stages and processes of self-change of smoking: toward an integrative model of change. Journal of consulting and clinical psychology, 51, 390-395.

Prochaska, J. O., \& DiClemente, C. C. (1986). Toward a comprehensive model of change. In W. R. Miller \& N. Heather (Eds.), Treating addictive behaviors. New York: Plenum.

Rathbun, R. C., Farmer, K. C., Stephens, J. R., \& Lockhart, S. M. (2005). Impact of an adherence clinic on behavioral outcomes and virologic response in the treatment of HIV infection: A prospective, randomized, controlled pilot study. Clinical Therapeutics, 27(2), 199-209.

Rawlings, M., Thompson, M., Farthing, C., Brown, L., Racine, J., Scott, R., et al. (2003). Impact of an educational program on efficacy and adherence with a twice-daily Lamivudine/Zidovudine/ Abacavir regimen in underrepresented HIV-infected patients. Journal of Acquired Immune Deficiency Syndrome, 34(2), 174-183.

Remien, R., Stirratt, M., Dolezal, C., Dognin, J., Wagner, G., Carballo-Dieguez, A., et al. (2005). Couplefocused support to improve HIV medication adherence: A randomized controlled trial. AIDS, 19(8), 807-814.

Reynolds, N., Testa, M., Su, M., Chesney, M., Neidig, J., Frank, 1., et al. (2008). Telephone support to improve Antiretroviral medication adherence a multisite, randomized controlled trial. Journal of the Association of Nurses in AIDS Care, 47(1), 62-68.

Rigsby, M. O., Rosen, M. I., Beauvais, J. E., Cramer, J. A., Rainey, P. M., O'Malley, S. S., et al. (2000). Cuedose training with monetary reinforcement: Pilot study of an antiretroviral adherence intervention. Journal of General Internal Medicine, 15, 841-847.

Rogers, P. G., \& Buliman, R. (1995). Prescription medicine compliance: a review of the baseline of knowledge- a report of the national council on patient information and education. Journal of Pharmacoepidemiology, 3(2), 3-36.

Rollnick, S., \& Miller, W. R. (2002). Motivational Interviewing: Preparing people for change. New York: The Guilford Press.

Roter, D. L., Hall, J. A., Merisca, R., Nordstrom, B., Cretin, D., \& Svarstad, B. (1998). Effectiveness of interventions to improve patient compliance. Medical Care, 36(8), 1138-1161. 
Rueda, S., Park-Wyllie, L. Y., Bayoumi, A. M., Tynan, A. M., Antoniou, T. A., Rourke, S. B., et al. (2006). Patient support and education for promoting adherence to highly active antiretroviral therapy for HIV/AIDS. Cochrane Database of Systematic Reviews(3), CD001442.

Safren, S., Hendriksen, E., Desousa, N., Boswell, S., \& Mayer, K. (2003). Use of an on-line pager system to increase adherence to antiretroviral medications. AIDS Care, 15(6), 787-793.

Safren, S. A., Otto, M. W., Worth, J. L., Salomon, E., Johnson, W., Mayer, K., et al. (2001). Two strategies to increase adherence to HIV antiretroviral medication: Life-Steps and Medication Monitoring. Behaviour research and therapy, 39(10), 1151-1162.

Scalera, A., Bayoumi, A. M., Oh, P., Risebrough, N., Shear, N., \& Tseng, A. I. (2002). Clinical and economic implications of non-adherence to HAART in HIV infection. Disease Management and Health Outcomes, 10(2), 85-91.

Schaalma, H., \& Kok, G. (2009). Decoding health education interventions: The times are a-changin'. Psychology and Health, 24(1), 5-9.

Schunk, D. H. (1996). Goal and self-evaluative influences during children's cognitive skill learning. American educational research journal, 33, 359-382.

Sendi, P., Bucher, H., Harr, T., Craig, B., Schwietert, M., Pfluger, D., et al. (1999). Cost effectiveness of highly active antiretroviral therapy in HIV-infected patients. AlDS, 13(9), 1115-1122.

Sethi, A. K., Celentano, D. D., Gange, S. J., Moore, R. D., \& Gallant, J. (2003). Association between adherence to antiretroviral therapy and Human Immunedeficiency Virus drug resistance. Clinical Infectious Diseases, 37, 1112-1118.

Sheeran, P. (2002). Intention-behavior relations: A conceptual and empirical review. European Review of Social Psychology, 12(1), 1-36.

Sheeran, P., \& Abraham, C. (2003). Mediator of moderators: Temporal stability of intention and the intention-behavior relation. Personality and Social Psychology Bulletin, 29(2), 205-215.

Sheeran, P., Abraham, C., \& Orbell, S. (1999). Psychosocial correlates of heterosexual condom use: A meta-analysis. Psychological Bulletin, 125(1), 90-132.

Simoni, J., Pantalone, D., Plummer, M., \& Huan, B. (2007). A randomized controlled trial of a peer support intervention targeting Antiretroviral medication adherence and depressive symptomatology in HIVpositive men and women. Health Psychology, 26(4), 488-495.

Simoni, J. M., Frick, P. A., \& Huang, B. (2006). A Longitudinal evaluation of a social support model of medication adherence among HIV-positive men and women on antiretroviral therapy. Health Psychology, 25(1), 74-81.

Simoni, J. M., Pearson, C. R., Pantalone, D. W., Marks, G., \& Crepaz, N. (2006). Efficacy of Interventions in Improving Highly Active Antiretroviral Therapy Adherence and HIV-1 RNA Viral Load: A MetaAnalytic Review of Randomized Controlled Trials. Journal of Acquired Immune Deficiency Syndromes, 43(S1), S23-S35.

Smith, S. R., Rublein, J. C., Marcus, C., Brock, T. P., \& Chesney, M. A. (2003). A medication selfmanagement program to improve adherence to HIV therapy regimens. Patient Education and Counseling, 50, 187-199.

Sniehotta, F. F., Scholz, U., \& Schwarzer, R. (2005). Bridging the intention-behaviour gap: Planning, selfefficacy, and action control on the adoption and maintenance of physical exerise. Psychology and Health, 20(2), 143-160.

Stewart, M. A. (1995). Effective Physician-patient communication and health outcomes: a review. Canadian Medical Association Journal, 9, 1423-1433.

Tam, L., Chui, C., Brumme, C., Bangsberg, D., Montaner, J., Hogg, R., et al. (2008). The relationship between resistance and adherence in drug-naive individuals initiating HAART is specific to Individual drug classes. Journal of Acquired Immune Deficiency Syndrome, 49(3), 266-271. 
Tuldra, A., Fumaz, C. R., Ferrer, M. J., Bayes, R., Arno, A., Balague, M., et al. (2000). Prospective randomized two-arm controlled study to determine the efficacy of a specific intervention to improve long-term adherence to highly active antiretroviral therapy. Journal of Acquired Immune Deficiency Syndromes, 25(3), 221-228.

Urquhart, J. (1997). The electronic medication event monitor - lessons for pharmacotherapy. Clinical Pharmacokinetics, 32, 345-356.

van Breukelen, G. J.P. (2006). ANCOVA versus change from baseline; more power in randomized studies, more bias in nonrandomized studies. . Journal of Clinical Epidemiology, 59, 920-925.

Van Empelen, P., \& Kok, G. (2008). Action-specific Cognitions of planned and preparatory behaviors of condom use among Dutch adolescents. Archives of Sexual Behavior, 37(4), 626-640.

van Houwelingen, H., Arends, L., \& Stijnen, T. (2002). Advanced methods in meta-analysis: Multivariate approach and meta-regression. Statistics in Medicine, 21(4), 589-624.

Van Servellen, G., Nyamathi, A., Carpio, F., D, P., Garcia-Teague, L., Herrera, G., et al. (2005). Effects of a treatment adherence enhancement program on health literacy, and adherence to HAART among low-income HIV-positive Spanish speaking Latinos. AIDS Patient Care and STDs, 19(11), 745-759.

Vrijens, B., \& Goetghebeur, E. (1997). Comparing compliance patterns between randomized treatments. Controlled Clinical Trials, 18, 187-203.

Wagner, G. J., \& Kanouse, D. E. (2003). Assessing usual care in clinical trials of adherence interventions for highly active antiretroviral therapy. Journal of Acquired Immune Deficiency Syndromes, 33, 276-277.

Wagner, G. J., Kanouse, D. E., Golinelli, D., Miller, L. G., Daar, E. S., Witt, M. D., et al. (2006). Cognitivebehavioral intervention to enhance adherence to antiretroviral therapy: a randomized controlled trial (CCTG 578). AIDS, 20, 1295-1302.

Weber, R., Christen, L., Christen, S., Tschopp, S., Znoj, H., Schneider, C., et al. (2004). Effect of individual cognitive behaviour intervention on adherence to antiretroviral therapy: prospective randomized trial. Antiviral Therapy, 9, 85-95.

Wendel, C. S., Mohler, M. J., Kroesen, K., Ampel, N. M., Gifford, A. L., \& Coons, S. J. (2001). Barriers to use of electronic adherence monitoring in an HIV clinic. Annals of pharmacotherapy, 35(9), 1010-1015.

Willett, J., \& Singer, J. D. (1988). Another cautionary note about $\mathrm{R}^{\wedge} 2$ : Its use in weighted least-squares regression analysis. American Statistician, 42(3), 236-238.

Wohl, A. R., Garland, W. H., Valencia, R., Squires, K., Witt, M. D., Kovacs, A., et al. (2006). A randomized trial of directly administered antiretroviral therapy and adherence case management intervention. Clinical Infectious Diseases, 42, 1619-1627.

Zimmerman, B. J. (2000). Attaining self-regulation. In M. Boekaerts, P. R. Pintrich \& M. Zeidner (Eds.), Handbook of self-regulation (pp. 13-39). London: Academic press.

Zimmerman, B. J., \& Kitsantas, A. (1996). Self-regulated learning of a motoric skill: The role of goal setting and self-monitoring. Journal of applied sport psychology, 8, 69-84.

Zimmerman, B. J., \& Martines-Pons, M. (1992). Perception of efficacy and strategy use in the selfregulation of learning. In D. H. Schunk \& J. Meece (Eds.), Student perceptions in the classroom: causes and consequences (pp. 185-207). Hillsdale, NJ: Erlbaum. 


\section{SUMMARY}

Since 1996, HIV can be effectively suppressed with Highly Active Antiretroviral Therapy (HAART). However, the effectiveness of HAART depends on how accurately patients take their medication. With 90-95\% adherence being considered sufficient for the long-term suppression of HIV, research shows that approximately half of the patients is insufficiently adherent. Behavioral interventions that effectively support patients in improving their adherence can therefore have a large impact on patients' health and quality of life. Despite that numerous intervention studies and several research syntheses have been conducted, scientific evidence for effective adherence care is rather poor. The main objective of the research described in this thesis was to expand the evidence-base for HIV-adherence care through the theoryand evidence-driven development and synthesis of adherence supporting interventions. Moreover, we addressed several theoretical and methodological issues relevant to the larger domain of behavioral science.

One line of research in this thesis focused on designing and testing a new, theory- and evidence-based adherence intervention (AIMS) designed to fit in HIVclinics' routine care procedures. AIMS was first pilot-tested (Chapter 2) and then studied more rigorously in a randomized controlled trial (Chapter 4). The studies showed that the adherence and viral suppression rates improved among patients receiving AIMS compared to control patients receiving high-quality standard care. Moreover, the intervention was evaluated positively by both the health care providers and the patients, and could largely be delivered during routine clinical visits. Hence, although AIMS has yet to be tested among non-Caucasian patients, the intervention seems to be a valuable and feasible addition to routine adherence care for HIV-infected patients.

In a third study, questionnaire data obtained from the control participants in the randomized controlled trial were used to address a current theoretical question in social science, namely the identification of factors that help explain the limited predictive value of people's behavioral intentions for their future behavior (Chapter 5). The results showed that certain instrumental adherence activities, like selfmonitoring of the whether one's medication intake is sufficient, mediated the intention-behavior relation, explaining a substantial proportion of variance in future behavior on top of intention. Hence, it seems instrumental acts can be a valuable addition to determinant studies, and possibly to behavioral theories that consider people's intentions as the most proximate predictor of their health behavior.

The second line of research described in this thesis consists of two metaanalytic studies that examined the impact of adherence care on adherence and viral suppression rates (Chapters 3 and 6 ). The research showed that both intervention 
and control groups in HAART adherence trials were exposed to highly effective forms of adherence care that explained up to $30-45 \%$ points in the observed viral suppression rates. Moreover, a number of important confounders of treatment effectiveness could be identified. We finally found that the effects obtained in HAART adherence intervention trials could be best explained by the content of the experimental interventions after controlling for the variability in care provided to the control groups in these trials. Hence, these studies showed how adherence care impacts medication intake behavior and treatment success rates. Moreover, the research points out several important population and methodological issues relevant for future (HAART) adherence research. Finally, these studies suggest that meta-analysts of behavior change interventions should control their analyses for the variability in care delivered to control groups in order to more accurately explain the observed intervention effects.

The clinical and meta-analytic studies point out several theoretical and methodological factors that are relevant to behavioral science in general. Moreover, the findings permit the development of evidence-based guidelines for highly effective adherence care in clinical practice. The wide-spread adoption and implementation of current best practice in Western countries is expected to have a large impact on patients' adherence and viral suppression rates. Moreover, the quality and effectiveness of current best practice could be further enhanced though the application of the AIMS intervention. Hence, also considering the feasibility of these practices, we hope these findings will not remain a scientific contribution only, but that they will also diffuse to clinical practice for the betterment of patients' health and quality of life. 


\section{SAMENVATTING}

HIV kan sinds 1996 effectief onderdrukt worden met behulp van een combinatie van antiretrovirale medicijnen (HAART). De effectiviteit van deze behandeling wordt echter voor een belangrijk deel bepaald door accuraat de medicijnen worden ingenomen. Op basis van de huidige richtlijn van $90-95 \%$ therapietrouw, blijkt dat ongeveer de helft van de patiënten de medicijnen niet voldoende inneemt. Succesvolle gedragsinterventies die patiënten helpen om beter hun medicijnen in te nemen, kunnen daarom een grote impact hebben op de gezondheid en kwaliteit van leven van Hiv-geïnfecteerde patiënten. Ondanks dat er al vele interventiestudies en verscheidende meta-analyses uitgevoerd zijn, is er nog maar weinig bewijs voor effectieve therapietrouwzorg. Het hoofddoel van het onderzoek dat is beschreven in deze these was om een bijdrage te leveren aan de wetenschappelijke basis voor effectieve therapietrouwzorg bij de behandeling van HIV. Daarnaast richten enkele onderzoeken zich ook op theoretische en methodologische onderwerpen die relevant zijn voor de gedragswetenschappen in het algemeen.

Eén onderzoekslijn die is beschreven in deze these betreft het ontwikkelen en testen van een nieuwe therapietrouwinterventie (AIMS). De AIMS interventie was ontwikkeld op basis van theorie en onderzoek, en ontworpen om binnen de reguliere HIV-zorg te passen. AIMS werd eerst bestudeerd in een kleinschalig vooronderzoek (Hoofdstuk 2), en vervolgens meer gedegen onderzocht in een gerandomiseerde studie (Hoofdstuk 4). De onderzoeken lieten zien dat de therapietrouw en virale onderdrukking verbeterden bij de patiënten die de AIMS interventie ontvingen vergeleken met de controlepatiënten die hoogkwalitatieve standaard zorg ontvingen. Bovendien werd de interventie positief geëvalueerd door de zorgverleners en de patiënten, en kon AIMS grotendeels tijdens de beschikbare tijd voor reguliere klinische bezoeken worden afgeleverd. Ofschoon AIMS nog onderzocht dient te worden onder niet-Kaukasische patiënten (lees: niet-Europese) patiënten, lijkt het dat de AIMS interventie een waardevolle en uitvoerbare toevoeging is aan de reguliere zorg voor HIV-patiënten.

In een derde onderzoek is gekeken naar vragenlijstgegevens van de controlepatiënten in de gerandomiseerde studie. Dit onderzoek richtte zich op een actueel theoretisch onderwerp in de sociale wetenschappen, namelijk het identificeren van factoren die helpen te verklaren waarom de intenties om een gedrag te vertonen zo beperkt voorspellen of mensen dat gedrag ook daadwerkelijk uitvoeren (Hoofdstuk 5). De resultaten van dit onderzoek laten zien dat bepaalde instrumentele activitejten, zoals het in de gaten houden of de medicatie-inname wel voldoende is, verklaarden hoe de gedragsintentie zich vertaalde in gedrag, en dat deze activiteiten een groot deel van de variantie in toekomstig gedrag voorspelden. Deze studie 
laat zien dat instrumentele activiteiten een belangrijke toevoeging zouden kunnen zijn aan studies die trachten gedrag te voorspellen en verklaren, en mogelijk aan gedragstheorieën waarin de gedragsintentie de meest directe voorspeller is van gedrag.

De tweede onderzoekslijn in deze these bestaat uit twee meta-analyses, waarin is gekeken naar de impact van therapietrouwzorg op therapietrouw en virale onderdrukking (Hoofdstukken 3 en 6 ). Uit deze onderzoeken is gebleken dat zowel interventie- als controlegroepen in HAART therapietrouw interventiestudies hoogeffectieve therapietrouwzorg ontvingen, en dat deze zorg tot $30-45 \%$ punten in virale onderdrukking verklaarde. Bovendien konden een aantal andere belangrijke factoren geïdentificeerd worden die van invloed waren op de effectiviteit van de behandeling. Ten slotte bleek dat de inhoud van de therapietrouwinterventies een goede voorspeller was van de geobserveerde interventie-effecten, indien er gecontroleerd werd voor verschillen in therapietrouwzorg die werd geleverd aan de controlegroepen in de studies. Deze onderzoeken laten de impact zien van therapietrouwzorg op de medicatie-inname en het aantal patiënten met een succesvole behandeling. Bovendien wijzen de onderzoeken verscheidene belangrijke populatie en methodologische factoren aan die relevant zijn voor toekomstig (HAART) therapietrouwonderzoek. Ten slotte laten deze studies zien dat meta-analyses van gedragsinterventies zouden moeten controleren voor verschillen in zorg geleverd aan controlegroepen om de interventie-effecten beter te kunnen verklaren.

De klinische en meta-analytische onderzoeken duiden verschillende theoretische en methodologische factoren aan die relevant zijn voor de gedragswetenschappen in het algemeen. Bovendien kunnen op basis van de gevonden resultaten wetenschappelijk onderbouwde richtlijnen voor hoogeffectieve therapietrouwzorg ontwikkeld worden. Het is te verwachten dat de grootschalige invoering van de huidige 'best practice therapietrouwzorg' in Westerse landen zal resulteren in een substantiële toename van het aantal patiënten met $95 \%$ therapietrouw en een volledige virale onderdrukking. Bovendien kan de kwaliteit van de huidige 'best practice' nog verder verhoogd worden met behulp van de AIMS interventie. We hopen daarom dat deze uitkomsten, ook met het oog op de praktische bruikbaarheid van deze interventies, niet alleen een wetenschappelijke bijdrage zullen vormen, maar dat ze ook gebruikt zullen worden in de dagelijkse gezondheidszorg voor de verbetering van de gezondheid en kwaliteit van leven van de mensen die zijn getroffen door HIV. 


\section{DANKWOORD}

Beste Harm en Gerjo- Ik kan wel wat boeken vol schrijven over wat ik van jullie geleerd heb.... Wat voor mij nog waardevoller is geweest, was de vrijheid en het vertrouwen dat ik van jullie kreeg om richting te geven aan dit proefschrift, terwijl ik daarbij altijd op jullie terug kon vallen als ik erachter kwam waar ik nu weer aan was begonnen...

Zeer veel dank!

Beste Jan: Ik ben je zeer erkentelijk voor het vertrouwen dat je me hebt geschonken in de afgelopen jaren, je blijvende enthousiasme voor dit onderzoek, en uiteraard je expert-input.

Beste Gerard, vergeleken met al jouw onderricht via een mail of 250 , valt die cursus 'Advanced statistics' in het niet. Merci bien!

Aan alle artsen van de HIV-poli op het AMC: Bedankt voor jullie input in en medewerking aan de interventiestudies.

Aan de HIV-consulenten Bonnie, Gert-Jan, Nicolette, Marian, en Ellen, aan Margje (maatschappelijk werk), en in het bijzonder aan de HIV-consulenten Hans van Eden, Astrid van Hes, Hans-Erik Nobel en Wiliam Koevoets: Ik wil jullie enorm bedanken voor al jullie inzet en inbreng die nodig was om de langdurige onderzoeken met succes uit te voeren. En dat bovenop alle "gewone" werkzaamheden.... En voor alle koffie, lunches, en de incidentele-maar memorabele- dinertjes en borrels!

Wolfgang, it's been a real pleasure working with you on these meta's. Hope to work with you on many more of these projects in the future.

Beste Jimmy, bedankt voor jouw uitermate geduldige en creatieve database-werk!

Geachte doktoren Hug, Jochen, GJ, Schoenmakers, en drs. Dikkie- Tsja... Ik kan er niets anders van bakken dan dat het een plezier is om met jullie bevriend te zijn geraakt. Uiteraard hebben die 365 inhoudelijke discussies ook wel het een en ander betekend voor de kwaliteit van dit proefschrift... Cheers!

Maartje S.: Dank voor de vele gesprekken, kaasplankjes en mooie wandelingen! DE manier om PhD-stoom af te blazen en weer in het hier en nu te komen....

Beste Onder Uns redactie: Met veel plezier denk ik terug aan onze maandelijkse sessies waar ik altijd naar uit keek, ook toen de druk begon toe te nemen. $U$ wordt gemist...

Rob, Sarah, Hans, Saskia, Marieke, Jill en andere (oud)medewerkers van de UNS5/40 die ik hier bij naam zou moeten noemen: het was een toffe tijd met $u$ ! 
Beste Herman, J j

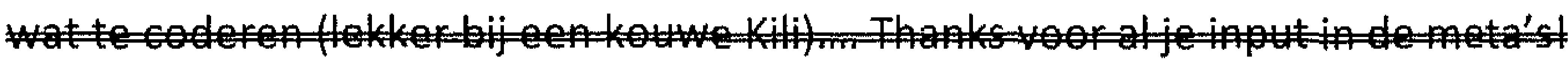

ik mis je emails, onze Afrika-, meta- en cultuur-plannen, de borrels aan keukentafels, je "zaken-in-perspectief-zetten", jouw enthousiasme en ervaring....

Maar vreemd genoeg hoor ik je nog wel regelmatig de meest ontnuchterende opmerkingen maken als ik me weer eens ergens druk over aan het maken ben....

Dear Charles, thanks for all the inspiring meta-discussions and input!

Beste co-Wageningers: Dank voor de steun en ruimte om dit tot een goed eind te brengen!

Bartos, ondanks dat we wat ver uit elkaar zaten: Een telefoongesprek van 10 minuten en ik had weer genoeg gelachen voor de hele week. Redelijk onbetaalbaar...

Lieve Wies en Walter, super-ouders, heel veel dank voor alle goede gesprekken, lekkere diners en wijntjes op Hoger einde, de boot, en het bemoedigend knikken bij al mijn verhalen. Gewoon, dat jullie er altijd voor me zijn.

Bro en sis, Roza en Merijn, Sammie en Skip: altijd heerlijk om bij jullie "uit te komen waaien". Family Matters....

Lieve Saskia, het afronden van dit proefschrift naast een nieuwe baan is niet alleen een uitdaging geweest voor mijn uithoudingsvermogen, maar volgens mij minstens net zoveel voor dat van jou. Echt super hoeveel je mij hierin hebt gesteund.

En: dank je voor al het andere dat jij brengt in mijn leven...

Ten slotte, ik ben de deelnemers aan de onderzoeken op de HIV-poli in het AMC zeer dankbaar voor de tijd en moeite die ze in de onderzoeken hebben gestoken. I would also like to express my gratitude to all the authors who made the metaanalyses possible by cooperating with our sizable requests for study information.

To all the patients, health care professionals and HIV-researchers who have been so kind to contribute their time, energy and expertise to these projects: I hope that the outcomes of these studies may be useful for your future research, be helpful in your clinical practice, and may ultimately contribute to improved health and quality of life of those affected by HIV/AIDS. 


\section{CURRICULUM VITAE}

Marijn de Bruin was born on October the $16^{\text {th }} 1977$ in Amsterdam, the Netherlands. He graduated from secondary school in 1996. After a year of travelling and working he started the study Health Sciences at the Maastricht University, the Netherlands. Between the start of his studies in September 1997 and his graduation in April 2004 (specialization Health Education and Health Promotion), Marijn studied, travelled for a year, and had various student-assistant positions (i.e., at the Faculty of Social Work, University of Sherbrooke, Canada; the Faculty of Health Sciences and the Faculty of Psychology, Maastricht University; and at the scientific department of Aardex Itd., (a medical device company)). Marijn then obtained a 1-year grant to conduct research for his master thesis. From this grant he was employed as a researcher at the Academic Medical Center (Amsterdam, the Netherlands) and conducted the pilot study described in this thesis. His master-thesis was approved in December 2003 and from February 2004 until September 2008 he did his Ph.D. research at the Faculty of Psychology, Maastricht University, the Netherlands.

Since September 2009, Marijn de Bruin has been employed as an assistantprofessor Communication Methods at the Social Sciences group, section Communication Science at the Wageningen University. 